\title{
Catálogo de la micobiota del Complejo Volcánico de Colima, México
}

\section{Catalogue of the mycobiota of the Colima Volcanic Complex, Mexico}

\section{Acta Botanica Mexicana}

\author{
Cesar Omar Torres Preciado' (D) , Olivia Rodríguez Alcántar’’2 (D) , María de Jesús Herrera-Fonsecal (D) , \\ Darío Figueroa-Garcíal
}

\section{Resumen:}

Antecedentes y Objetivos: El Complejo Volcánico de Colima (CVC) forma parte del Eje Volcánico Transversal Mexicano, localizado en el sureste de Jalisco y norte de Colima. Comprende cinturones de vegetación que cambian a través de un gradiente altitudinal desde 1200 hasta poco más de $4000 \mathrm{~m}$, en donde es posible observar bosques tropicales, bosques templados y zonas semiáridas, así como bosque mesófilo de montaña. Respecto a estudios previos sobre diversidad fúngica en esta región, solo se contaba con la cita de 140 especies registradas en los diferentes municipios que comprende el área del CVC. El objetivo del presente trabajo es actualizar el conocimiento de la micobiota registrada dentro de la zona de estudio.

Métodos: Se recolectaron ejemplares fúngicos durante los meses de junio a octubre entre 2011 y 2018. Adicionalmente, se realizó la revisión de material de la colección micológica del herbario IBUG. El estudio macro y micromorfológico se realizó con las técnicas convencionales en micología y el uso de bibliografía especializada para la determinación del material.

Resultados clave: Un total de 2082 ejemplares fueron determinados hasta especie, corresponden a 408 taxones; de ellos 360 son Basidiomycota y 48 Ascomycota. Se citan dos registros nuevos para la micobiota de México: Ductifera exidioidea y Psathyrella longistriata, y 11 para el estado de Jalisco: Bulgaria inquinans, Crepidotus applanatus, Gymnopilus sapineus, Hohenbuehelia spathulata, Hygrocybe spadicea, Inocybe obsoleta, Lepiota cristata, Pholiota lubrica, Rhizina undulata, Scleroderma aff. pseudostipitatum y Trichoderma alutaceum, mientras que para el estado de Colima se aportan 20 registros nuevos.

Conclusiones: Se considera importante incrementar estudios sistemáticos y exploraciones micológicas que permitan conocer más sobre este recurso, su posible uso y manejo racional, así como el análisis de especies endémicas o en riesgo y aspectos ecológicos que puedan mostrar la distribución actual y diversidad fúngica en dicha región.

Palabras clave: diversidad fúngica, especies endémicas, Jalisco, nuevos registros, taxonomía.

\section{Abstract:}

Background and Aims: The Colima Volcanic Complex (CVC) is part of the Transversal Mexican Volcanic Belt, located in the southeast of Jalisco and north of Colima. It includes vegetation belts that change through an altitude gradient ranging from 1200 to just over $4000 \mathrm{~m}$, where it is possible to observe tropical forests, temperate forests and semi-arid zones, as well tropical montane cloud forest. With respect to previous studies on fungal diversity in this region, only 140 species registered in the different municipalities that comprise the CVC area were cited. The objective of the present work is to update the knowledge of the mycobiota registered within the study area.

Methods: Fungal specimens were collected during the months of June to October between 2011 and 2018. Additionally, the fungal colection of the herbarium IBUG was revised. The macro- and micromorphological study was carried out with conventional mycological techniques and the use of specialized bibliography to identify the material.

Key results: A total of 2082 specimens were determined to species, corresponding to 408 taxa, of which 360 are Basidiomycota and 48 Ascomycota. Two new records are cited for the Mexican mycobiota: Ductifera exidioidea and Psathyrella longistriata, and 11 for the state of Jalisco: Bulgaria inquinans, Crepidotus applanatus, Gymnopilus sapineus, Hohenbuehelia spathulata, Hygrocybe spadicea, Inocybe obsoleta, Lepiota cristata, Pholiota lubrica, Rhizina undulata, Scleroderma aff. pseudostipitatum and Trichoderma alutaceum. For the state of Colima 20 new records are provided, which until now had not been cited.

Conclusions: It is considered important to increase systematic studies and mycological explorations that allow us to know more about this resource, its possible use and rational management, as well as the analysis of endemic or endangered species and ecological aspects that may show the current distribution and fungal diversity in this region.

Key words: endemic species, fungal diversity, Jalisco, new records, taxonomy.

'Universidad de Guadalajara, Departamento de Botánica y Zoología, Centro Universitario de Ciencias Biológicas y Agropecuarias (CUCBA), Laboratorio de Micología, Apdo. postal 1-139, Zapopan 45101, Jalisco, México.

${ }^{2}$ Autor para la correspondencia: olivia.rodriguez@ academicos.udg.mx
Recibido: 28 de febrero de 2020

Revisado: 23 de marzo de 2020.

Aceptado por Marie-Stéphanie Samain: 6 junio de 2020.

Publicado Primero en línea: 19 de junio de 2020.

Publicado: Acta Botanica Mexicana 127(2020).

cc) (i) Este es un artículo de acceso abierto (c) Atribución-No Comercial (CC BY-NC 4.0 Internacional).
Citar como: Torres Preciado, C. O., O. Rodríguez Alcántar, M. de J. Herrera-Fonseca y D. Figueroa-García. 2020. Catálogo de la micobiota del Complejo Volcánico de Colima, México. Acta Botanica Mexicana 127: e1686. DOI: 10.21829/abm127.2020.1686

e-ISSN: 2448-7589 


\section{Introducción}

Diversos estudios micobióticos se han realizado en los últimos 30 años en Jalisco, México. Sobresalen los de Nieves (1985), Téllez et al. (1988), Vázquez-González y GuzmánDávalos (1988), Rodríguez et al. (1994, 2010b, 2018), Fierros et al. (2000), Oliva-León (2000), Arroyo (2001), HerreraFonseca et al. (2002), Arceo-Orozco (2011), GándaraZamorano et al. (2014), Padilla-Velarde et al. (2016) y Terríquez-Villanueva et al. (2017). Particularmente en el Complejo Volcánico de Colima (CVC), zona que forma parte del Eje Volcánico Transversal Mexicano, reconocida por su alta riqueza, endemismos bióticos y porque constituye un área importante de especiación de organismos, sobresalen los trabajos de Fa y Morales (1993), Flores-Villela (1993), Ceballos et al. (1998), Espinosa et al. (2000) y SeguraTrujillo y Navarro-Pérez (2010).

Por su ubicación geográfica, el CVC se encuentra situado en un extremo de la zona de transición entre dos regiones biogeográficas como son la Neártica y la Neotropical, en donde es posible observar desde selvas tropicales a bosques templados y zonas semiáridas, así como la presencia de comunidades bióticas únicas como el bosque mesófilo de montaña (Rzedowski, 1978; Cox, 2001). Respecto a estudios previos sobre diversidad fúngica en el área del CVC, solo se contaba con la cita de 140 taxones registrados de los municipios que lo comprenden, recopilados la mayor parte por Sánchez-Jácome y GuzmánDávalos (2011) y de otros trabajos consultados como los de Bautista-Hernández et al. (2011), Landeros y GuzmánDávalos (2013) y Rodríguez et al. (2009, 2010a, 2013).

Con base en lo anterior, y por ser reconocido el Complejo Volcán Nevado de Colima como una zona de alta diversidad biológica, se considera relevante este estudio porque permite conocer la micobiota de esta región, la distribución geográfica de sus especies, sus aspectos ecológicos e importancia.

\section{Materiales y Métodos}

\section{Área de estudio}

El Complejo Volcánico de Colima (CVC) forma parte del Eje Volcánico Transversal Mexicano (Fig. 1), se localiza entre los estados de Jalisco y Colima, en las coordenadas geográficas $19^{\circ} 40^{\prime} 45^{\prime \prime} \mathrm{N}, 103^{\circ} 49^{\prime} 16^{\prime \prime} \mathrm{O}$ y $19^{\circ} 22^{\prime} 48^{\prime \prime N}, 103^{\circ} 25^{\prime} 32^{\prime \prime O}$, con una superficie total de $843.13 \mathrm{~km}^{2}$ (Magaña-Martínez y Palacios-Vargas, 2010; Segura-Trujillo y Navarro-Pérez, 2010). Dicho complejo está constituido por tres cumbres volcánicas: el Nevado de Colima (4270 m s.n.m.), el Volcán de Fuego (3860 m s.n.m.) y el Volcán del Cántaro (2900 m s.n.m.) (SEMARNAT-CONANP, 2007). EI CVC comprende seis municipios del estado de Jalisco: San Gabriel (Venustiano Carranza), Tonila, Tuxpan, Zapotiltic, Zapotitlán de Vadillo y Zapotlán el Grande (Ciudad Guzmán) y dos del estado de Colima: Comala y Cuauhtémoc (SEMARNAT-CONANP, 2007).

El complejo se localiza en un extremo de la zona de transición biogeográfica en la que es posible observar bosque tropical caducifolio y subcaducifolio, bosque mesófilo de montaña (principalmente en cañadas), bosque de Pinus-Quercus, bosque de Pinus hartwegii y bosque de Abies (Rzedowski, 1978; Jardel et al., 2014). La flora presenta cinturones de vegetación que cambian a través de un gradiente de altitud que va desde $1200 \mathrm{~m}$ hasta poco más de $4000 \mathrm{~m}$. El clima puede variar desde cálido y subhúmedo en las partes bajas, hasta frío subhúmedo en alta montaña, variación que está dada en función de su gradiente altitudinal y orientación geográfica (SeguraTrujillo y Navarro-Pérez, 2010). Los datos de precipitación media anual que se tienen dentro de los municipios que conforman el Complejo Volcánico de Colima, oscilan entre 700 y $800 \mathrm{~mm}^{3}$ con régimen de lluvias de junio a octubre (INIFAP, 2019).

\section{Recolección y determinación de material fúngico}

Se efectuaron diversas exploraciones a campo, al menos una por semana, durante el periodo de lluvias en los meses de junio a octubre entre 2011 y 2018; época de esporulación de la mayoría de los macromicetos considerados en este estudio.

Todo el material colectado fue herborizado y depositado en la Colección Micológica del Herbario IBUG de la Universidad de Guadalajara. Los ejemplares se determinaron a través del estudio de sus características macro y microscópicas basadas en el concepto de morfoespecie. Para el estudio micromorfológico se realizaron cortes del esporoma siguiendo las técnicas empleadas en micología propuestas por Largent et al. (1977) y Vellinga (1998). Los 


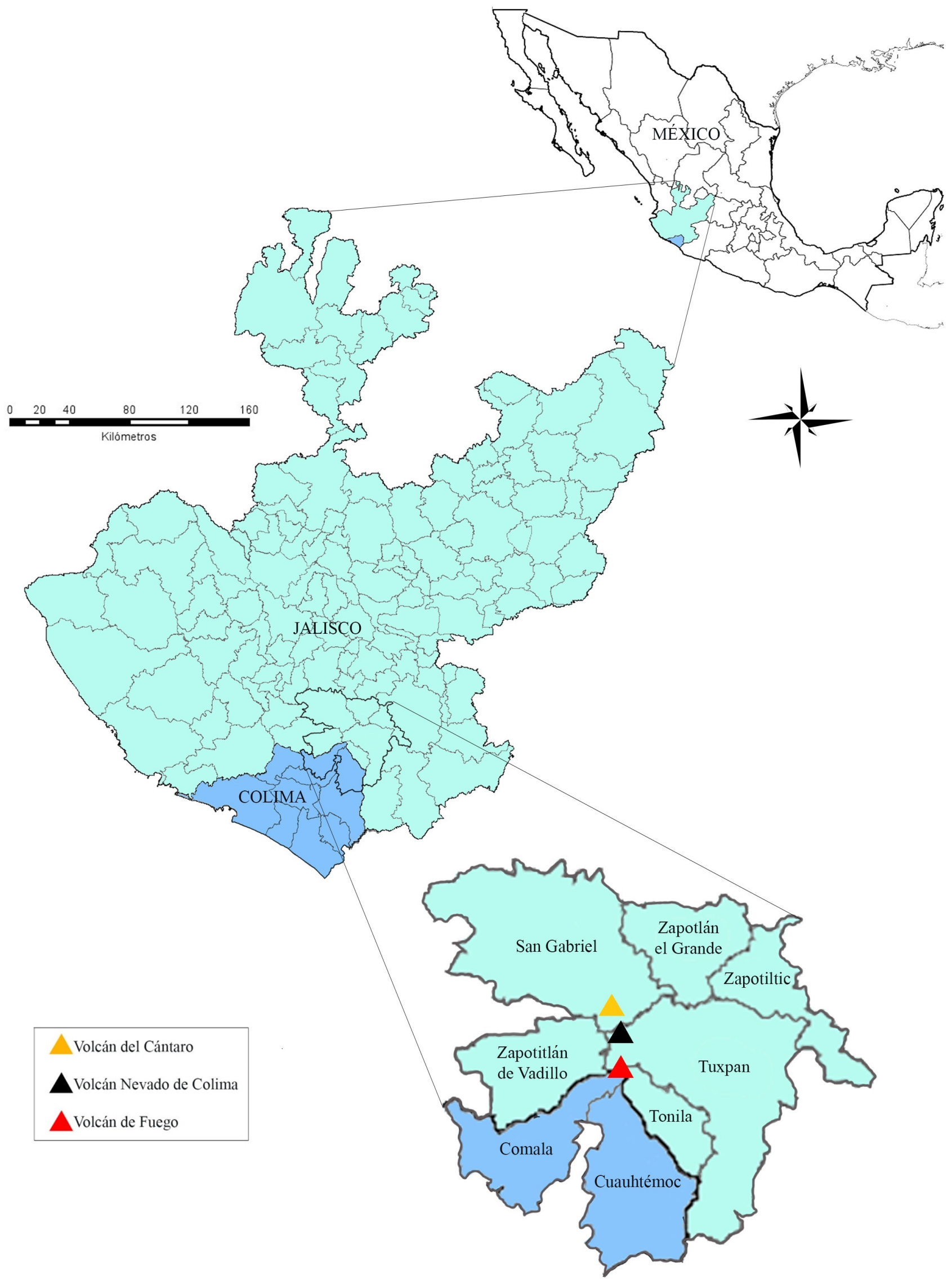

Figura 1: Localización del área de estudio Complejo Volcánico de Colima, México. 
especímenes fueron estudiados con un microscopio óptico (Zeiss K-7, Jena, Alemania). Dentro de algunas de las estructuras microscópicas con valor diagnóstico, están el tamaño y forma de las esporas, la cual, a partir de su medición, se obtiene el coeficiente $Q=$ largo/ancho cuyo intérvalo indica la forma básica de las esporas. Para la toma de las fotografías de los caracteres microscópicos se utilizó un microscopio óptico (Zeiss Axioskop 40, Jena, Alemania), cámara (AxioCam MRc, Zeiss, Jena, Alemania) y software Axio Vision 4 (Carl Zeiss Microscopy, 2020).

Aunado a lo anterior, se efectuó el estudio microscópico de ejemplares que estaban identificados como afínes, con el propósito de incrementar el número de registros y/o especies.

La determinación de las especies se realizó con base en la revisión de diversas obras y claves dicotómicas (Guzmán, 1977; Pegler, 1977, 1983, 1986; Breitenbach y Kränzlin, 1984, 1986, 1991, 1995, 2000; Pegler et al., 1995; Calonge, 1998; Kränzlin, 2005) y literatura especializada para cada grupo (Smith y Thiers, 1964; García-Jiménez y Castillo, 1981; Santiago et al., 1984; Gilberson y Ryvarden, 1986, 1987; Phillips, 1991, 2006; Vázquez-González y Guzmán-Dávalos, 1991; Valenzuela et al., 1994; GarcíaJiménez, 1999; Nuñez y Ryvarden, 2001; Rodríguez y Guzmán-Dávalos, 2001; Gándara-Zamorano y Ramírez-Cruz, 2005; Sánchez-Jácome y Guzmán-Dávalos, 2005; Groposo et al., 2007; Esqueda-Valle et al., 2009, 2011; CamachoSánchez, 2010; Raymundo et al., 2013a, b; Rodríguez, 2013; Montañez et al., 2016).

\section{Revisión de herbario y bibliografía}

Se revisó la colección micológica IBUG con el objetivo de incluir material no citado previamente en la literatura y confirmar la validación de los registros históricos con el fin de evitar sinonimias. Con base en el trabajo de SánchezJácome y Guzmán-Dávalos (2011) y revisión bibliográfica, se logró obtener la referencia completa de algunas especies citadas para dicha zona, la cual se menciona en el Apéndice 1.

\section{Listado de especies}

El listado actualizado de hongos se realizó con base en los ejemplares de las colecciones antes mencionadas, la revisión del material de herbario, la corroboración de algunas especies erróneamente identificadas de acuerdo con la base de datos pública digital de la Comisión Nacional para el Conocimiento y Uso de la Biodiversidad (CONABIO) (Guzmán-Dávalos, 2003), y de los taxa que previamente se tenían citados en la bibliografía del área de estudio.

El orden taxonómico de las especies se siguió de acuerdo con Wijayawardene et al. (2018), He et al. (2019), Index Fungorum (2019) y MycoBank (2019); consultándose estos últimos también para la validación de los taxones determinados, los nombres de autores y sinonimias. En la lista de especies (Apéndice 1) se incluye el nombre abreviado del colector, su número de colección, tipo de vegetación, localidad, hábitat e importancia.

En dicho listado, además de las especies estudiadas y corroboradas en este trabajo, se incluyen los taxones citados en la bibliografía y en el que la mayoría de los casos no se cuentan con el ejemplar y/o datos completos (o precisos) del material. La información sobre el uso de los hongos se obtuvo de la consulta bibliográfica de diversas obras (Guzmán, 1994, 2004, 2008; Hall et al., 2003; PérezMoreno et al., 2008; Shepard et al., 2008; Bautista-González y Moreno-Fuentes, 2014; Garibay-Orijel y Ruan-Soto, 2014). Se aporta la lista de colectores con su respectiva abreviatura (Apéndice 2) con un total de 136 registrados. Se incluye un listado de localidades de los municipios de ambos estados, Jalisco y Colima, que comprenden el CVC (Cuadro 1).

Para conocer el estado de riesgo de las especies o su estado de conservación se consultaron los documentos sobre normas y criterios nacionales e internacionales como la Unión Internacional para la Conservación de la Naturaleza (UICN, 2020) y la Norma Oficial Mexicana NOM-059-SEMARNAT-2020 (SEMARNAT, 2020). Toda la información obtenida fue agrupada y depurada en bases de datos diseñadas para éste y otros proyectos posteriores en formato Microsoft Excel $^{\circ} 2016$.

\section{Resultados}

Con base en el material colectado durante los años 20112018 que suman 1542, así como los 400 especímenes del herbario revisados y 140 de la bibliografía consultada, se tiene un total de 2082 ejemplares que fueron determinados 
Cuadro 1: Lista de localidades en la que fueron colectados los ejemplares estudiados. BMM=bosque mesófilo de montaña; $\mathrm{BP}=$ bosque de Pinus; $\mathrm{BPA}=$ bosque de Pinus con Abies; $\mathrm{BPE}=$ bosque de Pinus-Quercus; $\mathrm{BTC}=$ bosque tropical caducifolio; $\mathrm{BTSC}=$ bosque tropical subcaducifolio; $\mathrm{CM}=$ cultivo de maíz; R.M.O.=Repetidora de Microondas; $\mathrm{SD}=$ sin datos.

\begin{tabular}{|c|c|c|c|}
\hline Estado & Municipio & Localidad & Vegetación \\
\hline & & $\begin{array}{l}\text { 1) } 100 \mathrm{~m}-3 \mathrm{~km} \text { del crucero, El Floripondio camino a Las Víboras, Las Antenas, } \\
\text { R.M.O. }\end{array}$ & BPE \\
\hline & & 2) km 4-5 del crucero, El Floripondio camino a Las Víboras, Las Antenas, R.M.O. & BPE \\
\hline & & 3) km 6-7 del crucero, El Floripondio camino a Las Víboras, Las Antenas, R.M.O. & BPE \\
\hline & & 4) km 8-9 del crucero, El Floripondio camino a Las Víboras, Las Antenas, R.M.O. & $\mathrm{BP}$ \\
\hline & & $\begin{array}{l}\text { 5) km 5.5-6.5 camino hacia el Refugio La Joya, Parque Nacional Volcán Nevado } \\
\text { de Colima. }\end{array}$ & BPE \\
\hline & Lapotan el & 6) km 28 Refugio la Joya, Volcán Nevado de Colima. & BPA \\
\hline & & 7) Entre El Leoncito y Los Depósitos, Volcán Nevado de Colima. & $\mathrm{BP}$ \\
\hline & & 8) km 11.5 brecha El Fresnito, Antenas del Canal 13, Volcán Nevado de Colima. & BPE \\
\hline & & 9) km 14 brecha El Fresnito, Antenas del Canal 13, Volcán Nevado de Colima. & BPE \\
\hline & & 10) Parque los Ocotillos, Ciudad Guzmán. & BTC \\
\hline & & 11) Camino de Ciudad Guzmán a San Andrés Ixtlán. & BPE \\
\hline & & 12) Sin localidad precisa. & SD \\
\hline & & 13) Predio Los Corralitos. & BMM \\
\hline & & 14) Predio El Borbollón. & BMM \\
\hline & & 15) Camino al Volcán Nevado de Colima, ladera N. & BMM \\
\hline & 7anotitlán de Vadillo & 16) km 4.7 de la brecha El Milanés, Volcán Nevado de Colima. & BPE \\
\hline & 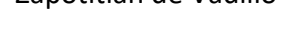 & 17) Poblado San José del Carmen. & BMM \\
\hline \multirow[t]{19}{*}{ Jalisco } & & 18) km 4 después del poblado de Telcruz, Predio La Colmena. & BPE \\
\hline & & 19) km 7-11 después del poblado de Telcruz, Predio La Colmena. & BPE \\
\hline & & 20) Sin localidad precisa. & SD \\
\hline & Zapotiltic & 21) Poblado El Rincón, km 1 camino al cerro El Calaque. & BTC \\
\hline & & 22) Cerro de Los Puercos. & BPE \\
\hline & Tuxnan & 23) $\mathrm{km} 2$ al S del poblado de Tuxpan. & BPE \\
\hline & & 24) Borde del Río Tuxpan. & BTSC \\
\hline & & 25) La Higuera. & $\mathrm{CM}$ \\
\hline & Tonila & 26) Volcán de Fuego ladera E, camino al poblado de Atenquique. & BPE \\
\hline & न & 27) Sin localidad precisa. & SD \\
\hline & & 28) 200 m-2 km sobre la brecha, Cerro Las Víboras, Puerto El Floripondio. & BPE \\
\hline & & 29) km 15 después de La Media Luna, Volcán Nevado de Colima. & BPE \\
\hline & & 30) Poblado El Apango, km 20 de la carretera Sayula-San Gabriel. & BPE \\
\hline & & 31) km 3 sobre la brecha que cruza el poblado de Apango. & BPE \\
\hline & San Gahriel & 32) Alrededores del poblado de San Gabriel. & BPE \\
\hline & & 33) Poblado La Mesa, 11 km al S de San Gabriel. & BPE \\
\hline & & 34) km 3 antes del poblado La Cañada, carretera Sayula-San Gabriel. & $\mathrm{BP}$ \\
\hline & & $\begin{array}{l}\text { 35) km } 8 \text { del crucero de La Yerbabuena-San Gabriel, carretera Tapalpa-San } \\
\text { Gabriel. }\end{array}$ & BPE \\
\hline & & 36) Sin localidad precisa. & SD \\
\hline \multirow{2}{*}{ Colima } & \multirow{2}{*}{ Comala } & 37) 500 m-1 km antes del poblado La Yerbabuena, camino a la Laguna María. & BMM \\
\hline & & 38) Alrededores de la Laguna María. & BMM \\
\hline
\end{tabular}


Cuadro 1: Continuación.

\begin{tabular}{|c|c|c|c|}
\hline Estado & Municipio & Localidad & Vegetación \\
\hline \multirow{4}{*}{ Colima } & \multirow{4}{*}{ Comala } & 39) km 1 camino La Becerra-La Yerbabuena, alrededores de la Laguna María. & BTC \\
\hline & & 40) km 9.5 después del poblado Campo Cuatro, camino hacia Lagunillas. & BPE \\
\hline & & 41) Cultivo de café, cercano a la carretera Comala-Colima. & BTSC \\
\hline & & 42) Laguna La María, ex hacienda de San Antonio. & BTC \\
\hline
\end{tabular}

hasta especie, lo que corresponde a 408 taxones. De estos, 48 se incluyen en la división Ascomycota y 360 en Basidiomycota; se encuentran incluidos en 204 géneros distribuidos en 84 familias. En cuanto al número de órdenes registrados, en Basidiomycota se adscriben 17 y cinco para Ascomycota. Los órdenes con mayor riqueza en especies de Basidiomycota fueron los Agaricales con 164 taxones, seguido por Polyporales (60), Boletales (43), Russulales (29) e Hymenochaetales (16). Las familias mejor representadas de Ascomycota y Basidiomycota, por el número de taxones y los géneros más ricos en especies, se incluyen en el Cuadro 2.

Se encontraron dos registros nuevos para México, Ductifera exidioidea Lowy y Psathyrella longistriata (Murrill) A.H. Sm., 11 para la micobiota del estado de Jalisco, Bulgaria inquinans (Pers.) Fr., Crepidotus applanatus (Pers.) P. Kumm., Gymnopilus sapineus (Fr.) Murrill, Hohenbuehelia spathulata (Pers.) Singer, Hygrocybe spadicea (Scop.) P. Karst., Inocybe obsoleta (Quadr. \& Lunghini) Valade, Lepiota cristata (Bolton) P. Kumm., Pholiota lubrica (Pers.) Singer, Rhizina undulata Fr., Scleroderma aff. pseudostipitatum Petch y Trichoderma alutaceum Jacklitsch y 20 especies como nuevos registros para Colima, de los cuales no se incluye su descripción dado que son muy conocidas, pero que hasta ahora no habían sido mencionadas.

La mayoría de los ejemplares incluidos en este estudio fueron colectados de Comala, Colima. Sin embargo, del municipio Cuauhtémoc, Colima, que también forma parte del CVC, nada se tiene reportado en la bibliografía consultada. Poco se conoce de la micobiota de Colima, hasta ahora 77 especies se tienen citadas, aunque sin dar localidades precisas (López-Ramírez y Medel, 2016), por lo que resultan importantes los nuevos registros aquí aportados, ya que se incrementará el conocimiento de la diversidad fúngica para esta entidad.

Cabe mencionar que del total de los 408 taxa determinados, 183 son exclusivamente registros nuevos para la zona, 140 habían sido previamente citados y 85 se citan por primera vez para algunos municipios, los cuales son señalados en el Apéndice 1. Los 183 taxones antes mencionados se encuentran distribuidos dentro de los municipios que conforman el CVC, siendoZapotlán el Grande (114) y San Gabriel (27) los que reportan un mayor número de especies para Jalisco, y cinco registros del estado de Colima del municipio Comala. Los taxa que se encontraron con mayor frecuencia y que fueron recolectados en más de cinco localidades fueron Auricularia nigricans (Sw.) Birkebak, Looney \& Sánchez-García, Lentinus tricholoma (Mont.) Zmitr., Schizophyllum commune Fr., Stereum ostrea (Blume \& T. Nees) Fr. y Trametes villosa (Sw.) Kreisel.

De acuerdo con los resultados, la diversidad fúngica registrada en la zona presentó una amplia distribución en los diferentes tipos de vegetación, siendo el bosque de Pinus-Quercus (BPE) el mejor representado con 363 taxones, seguido del bosque mesófilo de montaña (BMM) con 65, bosque de Pinus con elementos de Abies (BPA) con 50 y el bosque de Pinus (BP) con 45; mientras que en el bosque tropical caducifolio (BTC) y subcaducifolio (BTSC) se registraron 17 y cinco respectivamente, siendo estas dos últimas comunidades vegetales poco exploradas en el CVC.

Los resultados anteriores muestran que la mayor riqueza de especies fúngicas encontradas en el BPE se ha debido precisamente al sesgo en la recolección, situación algo similar a lo señalado en otros estudios (ej. Cifuentes et al., 1990; Fierros y Guzmán-Dávalos, 1995; HerreraFonseca et al., 2002; Gándara-Zamorano et al., 2014). Lo 
Cuadro 2: Riqueza de grupos taxonómicos registrados para el Complejo Volcánico de Colima, México.

\begin{tabular}{|c|c|c|c|c|}
\hline División & Órdenes & Familias & Géneros & Taxa \\
\hline \multirow{4}{*}{ Ascomycota } & Helotiales & 2 & 2 & 2 \\
\hline & Hypocreales & 3 & 4 & 7 \\
\hline & Leotiales & 2 & 2 & 2 \\
\hline & Pezizales & 7 & 16 & 29 \\
\hline \multirow{18}{*}{ Basidiomycota } & Xylariales & 2 & 3 & 8 \\
\hline & Agaricales & 22 & 68 & 164 \\
\hline & Auriculariales & 2 & 3 & 5 \\
\hline & Boletales & 9 & 22 & 43 \\
\hline & Cantharellales & 3 & 5 & 8 \\
\hline & Dacrymycetales & 1 & 3 & 5 \\
\hline & Geastrales & 1 & 1 & 6 \\
\hline & Gloeophyllales & 1 & 1 & 3 \\
\hline & Gomphales & 2 & 3 & 6 \\
\hline & Hymenochaetales & 2 & 7 & 16 \\
\hline & Phallales & 1 & 1 & 1 \\
\hline & Polyporales & 10 & 39 & 60 \\
\hline & Pucciniales & 2 & 3 & 3 \\
\hline & Russulales & 7 & 13 & 29 \\
\hline & Sebacinales & 1 & 2 & 3 \\
\hline & Thelephorales & 2 & 4 & 5 \\
\hline & Tremellales & 1 & 1 & 2 \\
\hline & Ustilaginales & 1 & 1 & 1 \\
\hline Total & 22 & 84 & 204 & 408 \\
\hline
\end{tabular}

anterior quizás se deba a la afinidad de las especies por determinado tipo de vegetación; esto tiene similitud con lo mencionado por Fierros et al. (2000), quienes señalan que cada comunidad vegetal puede presentar una micobiota característica, sumado al esfuerzo o intensidad de muestreo realizado.

De acuerdo con el Apéndice 1, se presenta el número de especies exclusivas de los principales tipos de sustrato, la mayoría de ellas son terrícolas (182 spp., 44.60\%), lignícolas $(161,39.46 \%)$, humícolas $(26,6.37 \%)$, fimícolas $(11,2.68 \%)$ y fungícolas $(7,1.71 \%)$. De las 182 especies terrícolas el mayor número registrado se adscribe al BPE con 112 y el de menor cantidad al BMM con tres. Respecto a las especies lignícolas, en general estas fueron recolectadas en $\mathrm{BPE}$, con un número de 74, mientras que en BMM y BPA se reportan siete y cuatro taxones, respectivamente; en tanto que en el BP y BTC se encontraron solo tres en cada uno de ellos.

Dentro de las especies humícolas, 18 son registradas del $\mathrm{BPE}$, en tanto que en BMM y BP se presentan cinco. Los taxones mejor representados son Gymnopus dryophilus (Bull.) Murrill, G. polyphyllus (Peck) Halling y Lepista nuda (Bull.) Cooke. Respecto a los hongos fímícolas, se registran siete taxones de BPE, y solo uno en BPA.

En cuanto a la importancia y uso de los hongos macroscópicos enlistados en el Apéndice 1, cabe destacar que 122 taxones son micorrizógenos, lo que representa $29.90 \%$ del total de especies registradas. Es importante mencionar que este tipo de asociación simbiótica micorrícica es más común en bosques templados que en tropicales en donde también puede presentarse, aunque de forma más escasa, quizás debido a las condiciones o factores medio ambienta- 
les (humedad y temperatura principalmente) y las especies de árboles presentes que sean susceptibles de micorrizarse (Guzmán-Dávalos y Guzmán, 1979; Janos, 1983).

Se registra un total de 127 hongos comestibles silvestres, entre ellos, Helvella crispa (Scop.) Fr., Hygrophoropsis aurantiaca (Wulfen) Maire, Hydnum repandum L., Laccaria laccata (Scop.) Cooke, Gymnopus dryophilus y Schizophyllum commune; estos dos últimos encontrados de forma frecuente en el área de estudio. La mayor riqueza de hongos comestibles se reporta para BPE con 118 especies (28.92\%), seguido del BMM con 23 $(5.63 \%)$ y $15(3.67 \%)$ en BP y BPA.

Asimismo, se citan 40 especies consideradas como venenosas, desde aquellas que causan ciertos problemas gastrointestinales hasta las venenosas mortales (RamírezTerrazo et al., 2014). Algunos de los taxones registrados para el CVC son Amanita gemmata (Fr.) Bertill, A. magnivelaris Peck, A. muscaria (L.) Lam., Chlorophyllum molybdites (G. Mey.) Massee, Hypholoma fasciculare (Huds.) P. Kumm., Inocybe geophylla (Bull.) P. Kumm., Lactarius torminosus (Schaeff.) Gray, Russula foetens Pers., Scleroderma areolatum Ehrenb. y $S$. texense Berk. Todos estos son micorrizógenos, a excepción de $C$. molybdites e $H$. fasciculare que son especies terrícola y lignícola, respectivamente. Dentro del grupo de los hongos medicinales se citan 82 taxones, entre los que se encuentran Ganoderma lobatum (Cooke) G.F. Atk., Phlebia tremellosa (Schrad.) Nakasone \& Burds., Schizophyllum commune, Trametes versicolor (L.) Lloyd y Tremella mesenterica Retz., todos ellos lignícolas. Referente a las especies parásitas, un total de 12 se registran, de las cuales cabe destacar a Heterobasidion annosum (Fr.) Bref. y Fomitopsis pinicola (Sw.) P. Karst. como hongos xilófagos causantes de enfermedades forestales (Ruiz Rodríguez y Pinzón-Picaseño, 1994; Cibrián-Tovar et al., 2007; Romero-Bautista et al., 2010).

A continuación, se presenta la descripción de los nuevos registros para Jalisco y México señalados con ** y - respectivamente, cuya simbología fue representada en el Apéndice 1. Por último, se incluyen láminas de imágenes, una de ellas con algunas de las especies colectadas (Fig. 2) y otra, con fotografías de basidiomas y estructuras microscópicas de los taxones Ductifera exidioidea y Psathyrella longistriata, citados por primera vez para el país (Fig. 3).

\section{Ascomycota}

Leotiomycetes

Leotiales

Bulgariaceae

** Bulgaria inquinans (Pers.) Fr., Syst. mycol. 2(1): 167. 1822. TIPO: encontrado sobre madera muerta de Quercus y Fagus (Espécimen tipo no designado, ni se menciona en cual herbario se depositó).

= Peziza polymorpha Oeder, Fl. dan.: t. 464. 1769.

इ Peziza inquinans Pers., Neues Magazin für die Botanik 1: 113. 1794.

$\equiv$ Ascobolus inquinans (Pers.) Nees, System der Pilze und Schwämme: 268, t. 39: 296. 1817.

इPhaeobulgaria inquinans (Pers.) Nannf., Nova Acta Regiae Societatis Scientiarum Upsaliensis 8(2): 311. 1932.

Apotecio en forma de copa, sésil, $10 \mathrm{~mm}$ de diámetro, consistencia gelatinosa, himenio liso, color negruzco; esporas (12.4-)13.2-15.2 × 6.4-7.2 $\mu \mathrm{m}, \mathrm{Q}=1.7-2.2$, elongadas a cilíndricas, en forma de rebanada de sandía, con una estría longitudinal hialina, en algunas con presencia de un cayo en ambos extremos, color verde-olivo.

Material examinado: MÉXICO. Jalisco, municipio Zapotlán el Grande, El Floripondio, camino a las antenas R.M.O., Las Víboras, faldas del Nevado de Colima, 12.VII. 1998, L. Arvizu Franklin 5 (IBUG).

Hábitat: lignícola, sobre madera podrida, gregario en bosque mesófilo de montaña; 2300 m s.n.m.

Distribución: en México ha sido registrada de Morelos (Bautista et al, 1986; Medel et al., 1999), Michoacán (Díaz-Barriga et al., 1988), Oaxaca (Raymundo et al., 2013b) e Hidalgo (Raymundo et al., 2019).

Notas taxonómicas: Bulgaria inquinans se distingue por su himenio liso, que se mancha de negro al tacto y por 


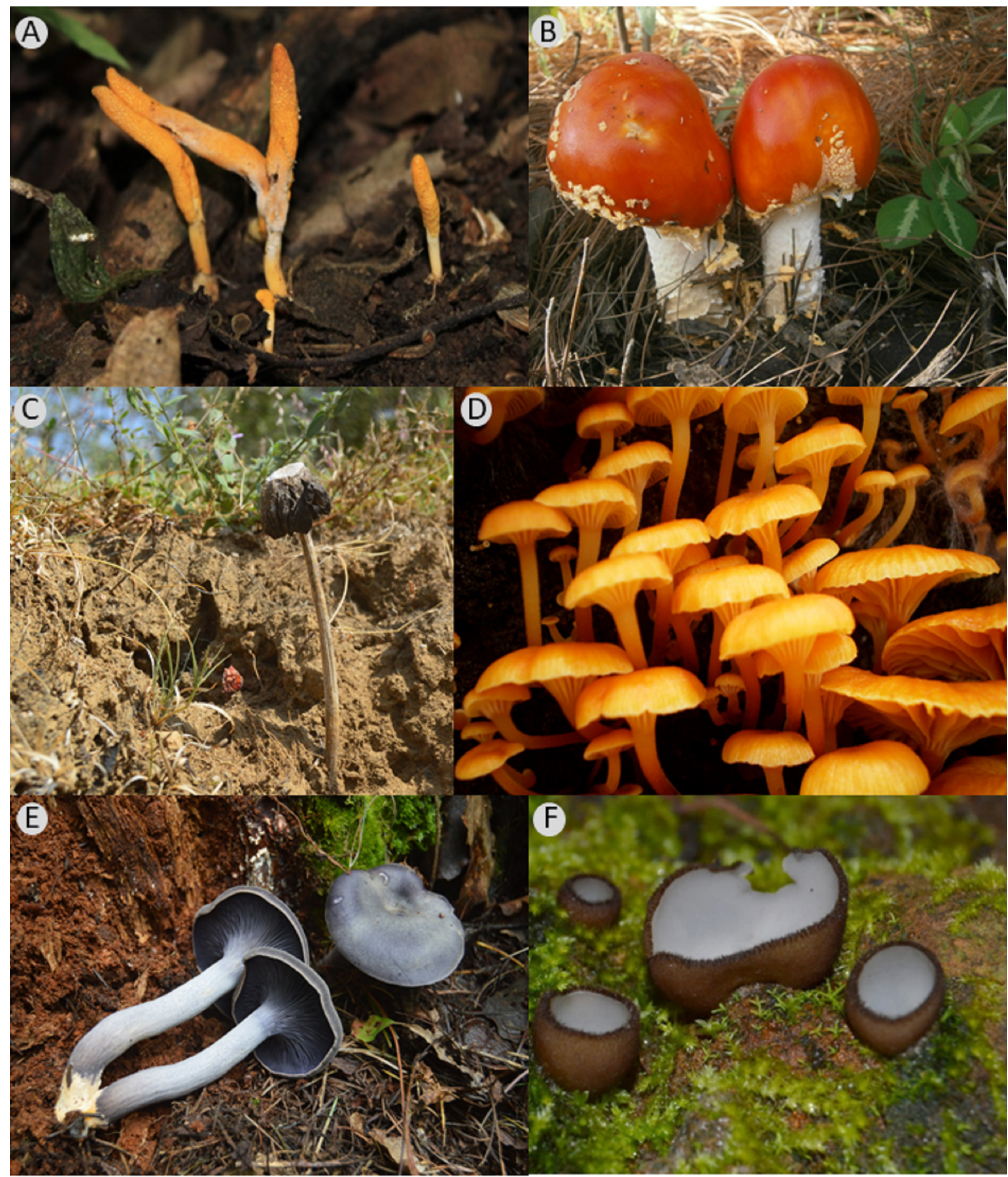

Figura 2: Cuerpos fructíferos de algunas especies de Ascomycota y Basidiomycota registradas dentro del Complejo Volcánico de Colima, México. A. Cordyceps militaris (L.) Fr.; B. Amanita muscaria (L.) Lam.; C. Montagnea arenaria (DC.) Zeller; D. Xeromphalina campanella (Batsch) Kühner \& Maire; E. Omphalotus mexicanus Guzmán \& V. Mora; F. Humaria hemisphaerica (F.H. Wigg.) Fuckel. Fotos: C. Torres-Preciado, D. Figueroa-García y D. Ulloa-Olmos. 


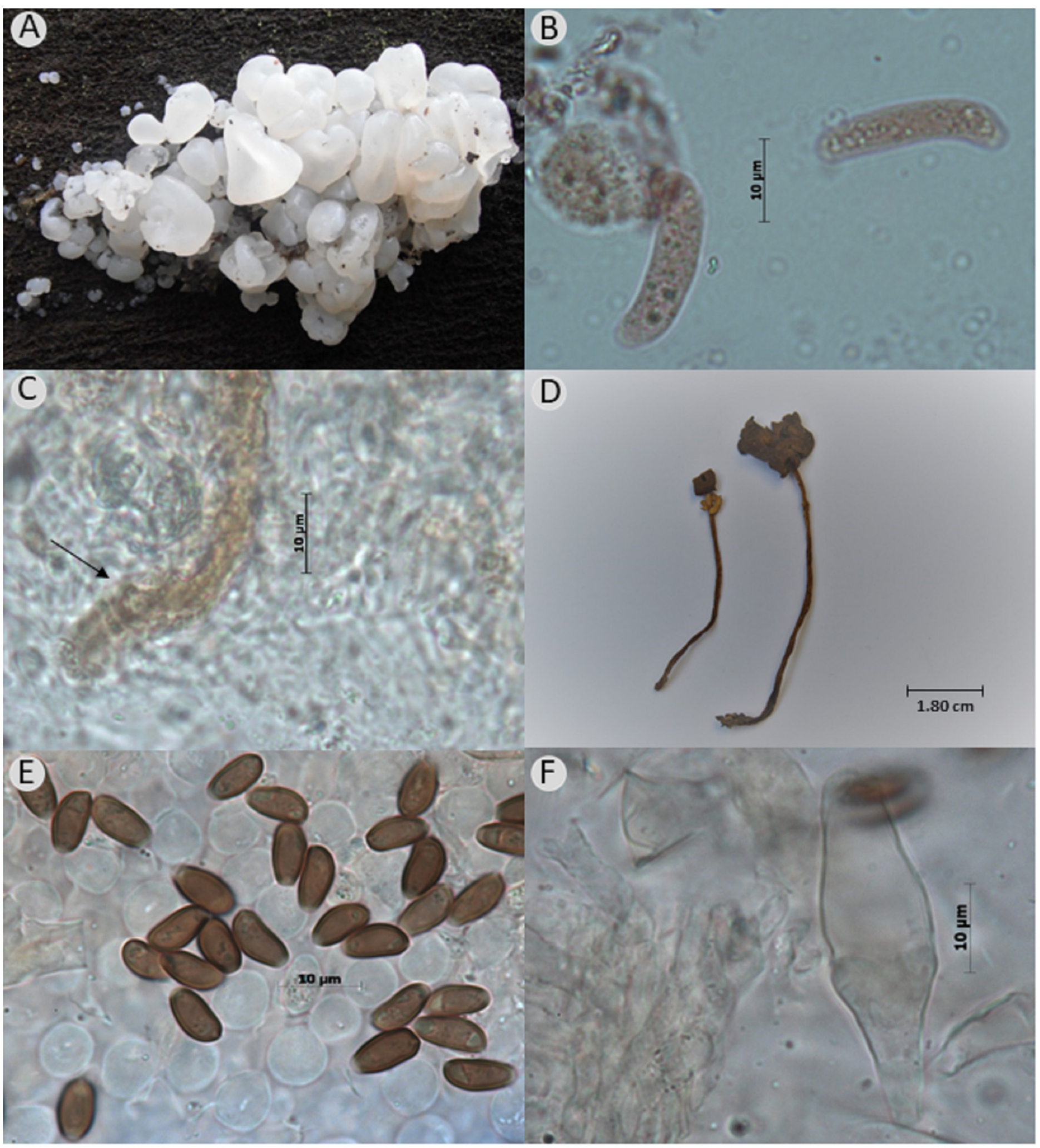

Figura 3: A. Ductifera exidioidea Lowy (C. Torres-Preciado 471, IBUG); B. esporas alantoides con ápice truncado; C. gleocistidio claviforme con ápice obtuso; D. basidiomas secos de Psathyrella longistriata (Murrill) A.H. Sm. (G. Nieves 276, IBUG); E. esporas elongadas con poro germinal hialino; F. pleurocistidios subutriforme. Fotos: C. Torres-Preciado, M. Herrera-Fonseca y D. Figueroa-García. 
las esporas color café oscuro. El material estudiado concuerda con lo señalado por Dennis (1981), Phillips (2006) y Beug et al. (2014). Por su parte Breitenbach y Kränzlin (1984) y Raymundo et al. (2019) describen esporas lisas, ampliamente elípticas en forma de limón o reniformes, 9$17 \times 6-7.6 \mu \mathrm{m}$, mientras que Dennis (1981) y Phillips (2006) las citan de 11-14 × 6-7 $\mu \mathrm{m}$, medidas que concuerdan con lo observado en el ejemplar revisado.

\section{Pezizomycetes}

Pezizales

\section{Rhizinaceae}

** Rhizina undulata Fr., Observationes mycologicae 1: 162. 1815. TIPO: en suelo arenoso (Espécimen tipo no designado, ni se menciona en cual herbario se depositó).

= Helvella inflata Schaeff., Fungorum qui in Bavaria et Palatinatu circa Ratisbonam nascuntur Icones 4: 102, t. 153. 1774.

$\equiv$ Racodium nigrum Schumach., Enumeratio Plantarum, in Partibus Sællandiae Septentrionalis et Orientalis Crescentium 2: 447. 1803.

इ Racodium vulgare Pers., Mycologia Europaea 1: 67. 1822.

Apotecio 3-5 cm de diámetro, sésil, margen irregular, con numerosos rizomorfos, color café-anaranjado a caférojizo negruzco, margen más claro blanquecino-amarillento; esporas (25-)26-31(-37) × 8.5-10 $\mu \mathrm{m}, \mathrm{Q}=(2.5-) 2.8-3.4(-4)$, cilíndricas a baseliformes, finamente rugosas, bigutuladas, color café-verdoso, con dos apéndices polares, 9-5 × 1-2 $\mu \mathrm{m}$, dextrinoides en melzer; ascas $400 \times 15-20 \mu \mathrm{m}$; paráfisis con ápice obtuso a subcapitado, contenido granuloso, muy abundante, color amarillento.

Material examinado: MÉXICO. Jalisco, municipio Zapotitlán de Vadillo, km 4.7 brecha El Milanés, Volcán de Colima, 11.XI.2007, A. Hermosillo 27 (IBUG).
Hábitat: humícola, gregario; bosque de PinusQuercus; 2337 m s.n.m.

Distribución: esta especie ha sido reportada para la micobiota mexicana de los estados de Morelos y Puebla (Chacón y Guzmán, 1983), del Estado de México (Frutis et al., 1985; Zarco, 1986), de Michoacán (Gómez Reyes et al., 2014). Se amplía su distribución al registrarse por primera vez de Jalisco.

Notas taxonómicas: el material estudiado concuerda con lo descrito por Dennis (1981), Breitenbach y Kränzlin (1984), Hansen y Knudsen (2000) y Yei-Zeng (2001). Solo difieren en el nombre de la forma que presentan las esporas. Dennis (1981) los Ilama apículos hialinos en cada extremo, Breitenbach y Kränzlin (1984) apéndices apuntados en ambos extremos, Hansen y Knudsen (2000) como apéndices polares puntiagudos, mientras que Yei-Zeng (2001) solo las describe con apículo grande. La morfología de las esporas es un carácter de importancia para la especie debido al tipo de éstas que presenta.

\section{Sordariomycetes}

\section{Hypocreales}

\section{Hypocreaceae}

** Trichoderma alutaceum Jaklitsch, Fungal Diversity. 48: 69. 2011. HOLOTIPO: Aislado de WU 29177 y depositado con el espécimen teleomorfo como cultivo seco WU 29177a.

इ Sphaeria alutacea Pers., Commentatio de Fungis Clavaeformibus: 12. 1797.

三 Cordylia alutacea (Pers) Fr., Observ. Mycol. 2: 317. 1818.

इXylaria alutacea (Pers.) Gray, A natural arrangement of British plants 1: 511. 1821.

इ Hypocrea alutacea (Pers.) Ces. \& De Not., Commentario della Società Crittogamologica Italiana 1(4): 370. 1863. 
三 Cordyceps alutacea (Pers.) Quél., Mémoires de la Société d’Émulation de Montbéliard sér. 2, 5: 487. 1875.

इ Podocrea alutacea (Pers.) Lindau, Nat. Pflanzenfamilien: 364. 1897.

$\equiv$ Podostroma alutaceum (Pers.) G.F. Atk. Bot. Gaz. 40: 416. 1905.

Estroma erecto, claviforme, formado por un pie color anaranjado a café amarillento en seco, de 3-7 × 1-2 cm, superficie irregular, aspecto pruinoso debido a los ostíolos, que se encuentran embebidos en todo el estroma; esporas 3-4 × 2-3 $\mu \mathrm{m}, \mathrm{Q}=1.1-2$, elipsoides, equinuladas, innamiloides, hialinas; ascas (35.1-)40-61.8(-66.5) × (2.8-)2.8-3.4 $(-3.6) \mu \mathrm{m}$, con 16 esporas, cilíndricas, inamiloides, hialinas.

Material estudiado: MÉXICO. Jalisco, municipio Zapotitlán de Vadillo, Predio La Colmena, km 7-11 después del poblado de Telcruz, ejido Zapotitlán, faldas del Nevado de Colima, 7.IX.2011, C. Torres-Preciado 336 (IBUG); municipio Zapotlán el Grande, km 8-9 del crucero, El Floripondio camino a Las Víboras, Las Antenas, R.M.O., 14.IX.2011, C. Torres-Preciado 376 (IBUG).

Hábitat: lignícola, solitario; bosque de Pinus-Quercus; 1999 m s.n.m.

Distribución: se amplía la distribución de Trichoderma alutaceum al registrarse de Jalisco, especie hasta ahora citada de Chiapas y Durango (Medel, 2002; Raymundo et al., 2012).

Notas taxonómicas: el ejemplar estudiado concuerda con lo descrito por Medel (2002), excepto que menciona esporas con un rango de $3.2-4(-4.8) \times 3.2(-4) \mu \mathrm{m}$, a diferencia del material revisado que son de menor tamaño, al registrarla de una selva alta perennifolia (Medel, 2002) y al no asociada a bosques de coníferas como lo citado por Dennis (1981). Así mismo se ajusta a las descripciones de Seaver (1910), Dennis (1981), Breitenbach y Kränzlin (1984) y Raymundo et al. (2012), solo que estos autores mencionan el estroma con tonalidades blanquecinas, amarillentas o marrón, a diferencia del material revisado que es de color anaranjado.

\section{Basidiomycota}

Agaricomycetes

Agaricales

Agaricaceae

** Lepiota cristata (Bolton) P. Kumm., Der Führer in die Pilzkunde: 137. 1871. TIPO: creciendo en en jardín de J. Cook, 15.IX.1787 (Espécimen tipo no designado, ni se menciona en cual herbario se depositó)

E Agaricus cristatus Bolton, An History of Fungusses, Growing about Halifax 1: 7. 1788.

三 Agaricus colubrinus var. cristatus (Bolton) Pers., Synopsis methodica fungorum 2: 259. 1801.

इ Lepiota colubrina var. cristata (Bolton) Gray, A natural arrangement of British plants 1: 602. 1821.

इ Lepiotula cristata (Bolton) E. Horak, Beiträge zur Kryptogamenflora der Schweiz 13: 338. 1968.

= Lepiota cristata var. pallidior Bon, Documents Mycologiques 11(43): 34. 1981.

三 Lepiota cristata var. macrospora (Zhu L. Yang) J.F. Liang \& Zhu L. Yang, Mycotaxon 116: 391. 2011.

Píleo 2-3 cm de diámetro, cónico, escamas aplanadas en toda la superficie, color café chocolate, umbo central del mismo color que las escamas; láminas libres, juntas, angostas, borde liso, color blanco-amarillento; estípite 4-5 $\times 1-2 \mathrm{~cm}$ de largo, central, uniforme, superficie estriadofibriloso, frágil, sólido, anillo supero, membranoso, colgante, concoloro al estípite y píleo; esporas (6.9-)7-9(9.5) $\times$ 3-4 $\mu \mathrm{m}, \mathrm{Q}=1.75-3(-3.6)$, cilíndricas a baseliformes, apice truncado, pared gruesa $(\geq 0.5 \mu \mathrm{m})$, gutuladas, contenido granuloso, hialinas; basidios (18-)19-22 × 6-7 $\mu \mathrm{m}$, claviforme a ventricosos, tetraspóricos, contenido refringente, fíbula basal, hialinos; pleurocistidios no observados; queilocistidios (21-)23-33 × 10-13 $\mu \mathrm{m}$, claviformes, pared gruesa $(1 \mu \mathrm{m})$, abundantes, hialinos; pileipellis de tipo himeniforme (20.9-)23.6-63(-77) × 1015.5(-17.7) $\mu \mathrm{m}$, claviformes, fíbula basal, hialinos. 
Material estudiado: MÉXICO. Jalisco, municipio Zapotlán el Grande, km 5.7 del camino que va hacia las Joyas, Parque Nacional Nevado de Colima, 17.VIII.2011, C. TorresPreciado 207 (IBUG).

Hábitat: terrícola, gregario; bosque de PinusQuercus; 2320 m s.n.m.

Distribución: especie de amplia distribución, pero no común, registrada hasta ahora del hemisferio norte, en Europa, Estados Unidos de América, Japón y México. Para la micobiota mexicana se había reportado de Colima y Veracruz (Murrill, 1911), del Estado de México, Hidalgo, Michoacán, Nuevo León (Valenzuela et al., 1981) y de Oaxaca (Villarruel-Ordaz et al., 2015).

Notas taxonómicas: Lepiota cristata se distingue por presentar una pileipellis himeniforme y esporas espolonadas, y macroscópicamente por el píleo umbonado con tonos café-rojizo de sus escamas concéntricas sobre fondo blanco. El ejemplar examinado concuerda con lo señalado por Murrill (1911), Valenzuela et al. (1981), Breitenbach y Kränzlin (1995) y Vellinga (2001). Se diferencia nuestro material de lo observado por Valenzuela et al. (1981), quienes describen queilocistidios más grandes (24$45 \times 7.5-13.5 \mu \mathrm{m})$ y de pared delgada. Vellinga (2001), por su parte, describe esporas espolonadas, de tamaño más pequeño, (5-)5.5-8.5(-10) × 2.5-3.5 $\mu \mathrm{m}$, y una pileipellis de tipo himeniforme. En tanto Breitenbach y Kränzlin (1995) describen esporas truncadas en forma de bala, 5.9-9.3 $\times$ 2.7-3.7 $\mu \mathrm{m}$ y una pileipellis himeniforme con elementos claviformes a cilíndricos.

\section{Hygrophoraceae}

** Hygrocybe spadicea (Scop.) P. Karst. (como 'Hydrocybe'), Bidr. Känn. Finl. Nat. Folk 32: 237. 1879. TIPO: Espécimen tipo no designado, ni se menciona en cual herbario se depositó.

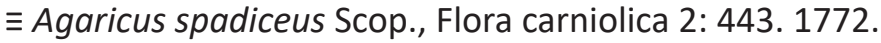

इ Hygrophorus spadiceus (Scop.) Fr., Epicrisis Systematis Mycologici: 332. 1838.
三 Hydrocybe spadicea P. Karst., Bidrag till Kännedom av Finlands Natur och Folk 32: 237. 1879.

三 Godfrinia spadicea (Scop.) Herink, Sborník Severoceského Musea 1: 66. 1958.

Píleo 41-43 mm de diámetro, cónico, cúspide, muy conspicuo, subviscoso, margen ondulado, color café oscuro en el centro, el resto irregularmente con zonas más claras; láminas subadheridas, subjuntas, borde erodado, color amarillas con tonos mamey; estípite 60-90 × 1-3 $\mathrm{mm}$, central, flexuoso hacia la base, superficie fibrilosa, subviscosa, hueco, color amarillo-verdoso a café muy claro; olor muy desagradable; esporas 10.4-12.4 × 6-7.6 $\mu \mathrm{m}$, $\mathrm{Q}=1.5-1.8$, elipsoides a elongadas, ápice obtuso y apículo evidente, pared delgada, hialinas; pileipellis un ixocutis, hifas 2.4-3.2 $\mu \mathrm{m}$ de diámetro, pared delgada, septadas, fíbulas y pigmento intracelular, color pardo-amarillento.

Material examinado: MÉXICO. Jalisco, municipio Venustiano Carranza, $3 \mathrm{~km}$ antes de La Cañada, carretera Sayula - Venustiano Carranza, 23.VII.1991, M. R. SánchezJácome 107 (IBUG).

Hábitat: humícola, gregario; bosque de Pinus; 2000 m s.n.m.

Distribución: con distribución en Europa y Estados Unidos de América (Arnolds, 1990). Se amplía su distribución en México al citarse para Jalisco, ya que solo se conocía del Estado de México (Santillán y Valenzuela, 1986).

Notas taxonómicas: Hygrocybe spadicea se caracteriza por el color del píleo y el tamaño de sus esporas. El material examinado concuerda con lo mencionado por Santillán y Valenzuela (1986) y Arnolds (1990). Este último describe esporas más grandes, 8-12(-13.5) × 5-9 $\mu \mathrm{m}$, que lo observado en el ejemplar estudiado, 10.4-12.4 $\times$ 6-7.6 $\mu \mathrm{m}$. Por su parte, Santillán y Valenzuela (1986) mencionan esporas ligeramente más pequeñas, 8-11(-12) × (4.8-)5.6-7.2 $\mu \mathrm{m}$. Estos últimos autores señalan además a Hygrocybe immutabilis (Peck) Murrill como una especie afín; sin embargo, su píleo es de color café amarillento a café verdoso. 
Hymenogastraceae

** Gymnopilus sapineus (Fr.) Murrill, Mycología 4(5): 254. 1912. TIPO: Espécimen tipo no designado, ni se menciona en cual herbario se depositó.

इ Agaricus hybridus Sowerby, Col. fig. Engl. Fung. Mushr. 2(18): tab. 221. 1799.

三 Agaricus penetrans Fr., Observationes mycologicae 1: 23. 1815.

इAgaricus sapineus Fr., Systema Mycologicum 1: 239. 1821. 三 Agaricus liquiritiae subsp. sapineus (Fr.) Pers., Mycologia Europaea 3: 184. 1828.

इ Flammula sapinea (Fr.) P. Kumm., Der Führer in die Pilzkunde: 82. 1871.

इ Gymnopilus penetrans (Fr.) Murrill, Mycologia 4(5): 254. 1912.

E Gymnopilus sapineus (Fr.) Maire, Fungi Catalaunici: Contributions à l'étude de la Flore Mycologique de la Catalogne: 96. 1933.

Píleo 3-5 cm de diámetro, plano-convexo, margen entero, superficie seca, escamoso, escamas aplanadas, color café; láminas adheridas, juntas, anchas, borde serrado a crenado, color amarillo ferruginoso; estípite 1-2 $\times$ 0.7-1.2 cm, central, uniforme, fibriloso, firme, sólido, micelio algodonoso basal, color blanco-amarillento; restos de velo presente; contexto suave, color amarillo, olor fúngico; $\mathrm{KOH}$ mancha el píleo de color café-rojizo obscuro a negruzco en seco; esporas 8-8.8 $\times$ 5.2-6 $\mu \mathrm{m}, \mathrm{Q}=1.4-1.6$, elipsoides, verrugosas, verrugas medianas, ápice subagudo a agudo, sin placa, dextrinoides, color anaranjadoamarillento; pleurocistidios no observados; queilocistidios 27.2-42.4 × 6.8-8.8 $\mu \mathrm{m}$, langeniforme-subutriformes, ápice subcapitado-capitado, fíbula basal, hialinos o contenido amarillento; trama himenófora subparalela, hifas septadas, con fibulas, hialinas a amarillentas; hifas laticíferas presentes, tortuosas, color anaranjado-amarillentas; trama del píleo radial, compacta, hifas de pared delgadasubgruesa, color amarillentas; pileipellis un cutis, hifas con bandas conspicuas, septadas, fíbulas presentes, pigmento incrustado; caulocistidios 48-59.2 × 3.6-4.8 $\mu \mathrm{m}$, cilíndricos, ápice capitado, con o sin contenido amarillento.
Material examinado: MÉXICO. Jalisco, municipio de Zapotlán el Grande, 100 m a 3.5 km después del crucero El Floripondio camino a Las Víboras, Las antenas R.M.O. faldas del Nevado de Colima, 13.XI.2012, C. Torres-Preciado 570 (IBUG).

Hábitat: lignícola, gregario; bosque de Pinus-Quercus con presencia de Abies Mill., 2200-2450 m s.n.m.

Distribución: Gymnopilus sapineus es una especie de amplia distribución de acuerdo con lo citado en la bibliografía, se conoce de Estados Unidos de América, Sudamérica, Europa, África, Asia y Australia; para México solo se conocía del estado de Oaxaca (Guzmán-Dávalos, 1996).

Notas taxonómicas: la especie aquí descrita se caracteriza por el color y ornamentación del píleo, y la trama radial de éste. El ejemplar se determinó según lo descrito por Hesler (1969) y Guzmán-Dávalos (1996), quienes la citan con presencia de caulocistidios capitados, velo presente, con píleo finamente escamoso e hifas de la pileipellis con pigmento en bandas, caracteres observados en el material revisado.

\section{Inocybaceae}

** Crepidotus applanatus (Pers.) P. Kumm., Der Führer in die Pilzkunde: 74. 1871. NEOTIPO: Loch Lomond, en tocón de madera, 27.VIII.1963, E. Kits van Waveren (L 986.062-019).

$\equiv$ Agaricus applanatus Pers., Observationes mycologicae 1: 8. 1796.

इ Agaricus planus Pers., Synopsis methodica fungorum: 480. 1801.

इCrepidotus globiger (Berk.) Sacc., Syll. Fung. 5: 879. 1887. $\equiv$ Crepidotus putrigenus (Berk. \& M.A. Curtis) Sacc., Syll. Fung. 5: 883. 1887.

$\equiv$ Crepidotus applanatus var. subglobiger Singer, Beih. Nova Hedwigia 44: 478. 1973.

Píleo 1-4.5 cm de diámetro, conchado o petaloide, 
liso, margen estriado cuando joven, color blanco a marrón pálido; láminas en forma radiada, poco separadas, onduladas, color blanquecino a ocre; esporas 4.5-7.8(-9) $\times$ 4-7(-7.2) $\mu \mathrm{m}, \mathrm{Q}=1-1.27(-1.28)$, en su mayoría globosas a subglobosas, algunas ampliamente elipsoides, equinuladas, gutuladas, apículo prominente, pared gruesa $(0.79 \mu \mathrm{m})$, hialinas; pleurocistidios no observados; queilocistidios 14$29(-30.8) \times 6-8(-10) \mu \mathrm{m}$, claviformes a cilíndricos, pocos lecitiformes, en su mayoría con ápice subcapitado a obtuso, hialinos, contenido refringente y fíbula basal.

Material examinado: MÉXICO. Jalisco, municipio Zapotlán el Grande, km 6.4 del camino que va hacia las Joyas, Parque Nacional Nevado de Colima, 17.VIII.2011, C. Torres-Preciado 218 (IBUG).

Hábitat: lignícola, solitario a gregario; bosque de Pinus-Quercus; 2334 m s.n.m.

Distribución: Crepidotus applanatus se tiene registrada de Europa, Estados Unidos de América y Asia (Breitenbach y Kränzlin, 2000). Se amplía su distribución al citarse de Jalisco, ya que solo se conocía de los estados de Chiapas y Oaxaca (Bandala y Montoya, 2008).

Notas taxonómicas: de acuerdo con lo mencionado por Bandala y Montoya (2008), una especie afín es Crepidotus cuneiformis Pat., de la cual se distingue por el tamaño más pequeño y forma de sus esporas, 5.5-7 × 5.5$6.5(-7) \mu \mathrm{m}, \mathrm{Q}=1.03$, globosas a subglobosas equinuladas, pero se puede diferenciar por la ausencia de pleurocistidios y la presencia de queilocistidios lageniformes y capitados. Por otra parte, Breitenbach y Kränzlin (2000) mencionan que la especie es casi imposible de determinar macroscópicamente, debido a la diferente coloración que presenta durante su desarrollo. Se puede confundir macroscópicamente con Crepidotus mollis (Schaeff.) Staude, pero se diferencia de ésta por el tamaño y forma de las esporas, 6.5-9.2 × 4.8-6.4 $\mu \mathrm{m}$ y queilocistidios vs. 30-60 × 5-9 $\mu \mathrm{m}$ cilíndricos a legeniformes.

** Inocybe obsoleta (Quadr. \& Lunghini) Valade, Index fungorum 165: 1. 2014. HOLOTIPO: FRANCIA. Dept.
Seine-et-Oise: Yerves, Chateau de la Grange, 18.VIII.1942, Romagnesi 42.59, herb. Romagnesi (PC).

I Inocybe obsoleta Romagn., Flora Analytique des Champignons Supérieurs: 218. 1953.

= Inocybe rimosa var. obsoleta Quadr. \& Lunghini, Quaderni dell’Accademia Nazionale dei Lincei 264: 109. 1990.

Píleo $4 \mathrm{~cm}$ de diámetro, cónico, umbo central, margen estriado-plicado, color café-crema; láminas subadheridas, juntas, angostas, borde entero, concoloro al píleo; estípite $7 \times 1-2 \mathrm{~cm}$ central, uniforme, superficie estriado-flocoso, frágil, sólido, concolor al píleo; esporas 10.1-10.9 × 6-6.8 $\mu \mathrm{m}, \mathrm{Q}=1.5-1.8$, ampliamente elipsoides a elongadas, lisas, pared gruesa $(\geq 0.4 \mu \mathrm{m})$, gutuladas, contenido refringente, coloración café-amarillento; basidios 35-40 × 10-11.8 $\mu \mathrm{m}$, claviformes, tetraspóricos, hialinos, fíbula basal; pleurocistidios ausentes; queilocistidios 21-54(-55) × 8-19 $\mu \mathrm{m}$, claviformes, cilíndricos a lageniformes, fíbula basal, hialinos.

Material estudiado: MÉXICO. Jalisco, municipio Zapotlán el Grande, 100 m después del crucero, El Floripondio, camino a las antenas R.M.O., Las Víboras, faldas del Nevado de Colima, 24.VIII.2011, C. Torres-Preciado 262 (IBUG).

Hábitat: terrícola, solitario a gregario; bosque de Pinus-Quercus; 2362 m s.n.m.

Distribución: es una especie registrada de Europa y Estados Unidos de América (Kuyper, 1986; Larsson et al., 2009; Fernández-Sasia, 2013). De México se tiene citada del Estado de México (Zarco, 1986; Pérez-Silva et al., 2011) y de Sonora (Pérez-Silva et al., 2006).

Notas taxonómicas: Inocybe obsoleta se caracteriza macroscópicamente por la presencia de un velo blanco o blanquecino en basidiomas jóvenes y la ausencia de olor, y microscópicamente por el tamaño pequeño de las esporas. Es afín a Inocybe rimosa (Bull.) P. Kumm.; sin embargo, se diferencia por los tonos amarillo oliváceo de sus láminas, el olor espermático y la presencia de esporas más grandes, 9.6-14 × 5.4-7.5 $\mu \mathrm{m}$ (Breitenbach y Kranzlin, 2000). La espe- 
cie aquí descrita ha sido considerada coespecifica o como simple forma o variedad de Inocybe rimosa. No obstante, de acuerdo con estudios moleculares realizado por Larsson et al. (2009), son dos especies distintas. El material estudiado concuerda con lo descrito por Kuyper (1986) y FernándezSasia (2013).

\section{Pleurotaceae}

** Hohenbuehelia spathulata (Pers.) Singer, Fieldiana Botany 21: 42. 1989. TIPO: Espécimen tipo no designado, ni se menciona en cual herbario se depositó.

इ Agaricus spathulatus Pers., Synopsis methodica fungorum 2: 479.1801.

इ Agaricus petaloides var. spathulatus (Pers.) Fr., Systema Mycologicum 1: 183. 1821.

$\equiv$ Pleurotus petaloides var. spathulatus (Pers.) P. Karst., Meddn Soc. Fauna Flora fenn. 14: 85. 1887.

三 Pleurotus spathulatus (Pers.) Peck, Annual Report on the New York State Museum of Natural History 39: 63. 1887. = Agaricus petaloides Bull., Herbier de la France 5: tab. 226. 1785.

= Hohenbuehelia petalodes (Bull.) Schulzer, Verhandlungen der Zoologisch-Botanischen Gesellschaft Wien 16: 45. 1866.

Píleo 5-20 mm de diámetro, forma conchada o petaloide, superficie ligeramente tomentosa, margen estriado, arqueado, color café; láminas decurrentes juntas, angostas, borde entero, color café más claro hacia el borde; estípite 1-3 $\times 0.7 \mathrm{~cm}$, lateral, superficie estriada longitudinalmente, consistencia flexible, color café; olor y sabor fúngico; esporas (4.1-)4.3-6.2(-6.5) × 3.5-3.7(-5.1) $\mu \mathrm{m}, \mathrm{Q}=1.07-1.31(-1.38)$, globosas a subglobosas hasta ampliamente elipsoides, apículo prominente, contenido refringente, hialinas, inamiloides; basidios 23-26(-30) × 5-7 $\mu \mathrm{m}$, tetraspóricos, claviformes, hialinos; cistidios 44-53(56) $\times 9-16 \mu \mathrm{m}$, metuloides con presencia de cristales en el ápice, setiformes a fusiformes, pared gruesa ( $\geq 0.5 \mu \mathrm{m})$, ápice rostrado, abundantes, coloración verdosa.

Material examinado: MÉXICO. Jalisco, municipio Zapotlán el Grande, 4 km después del crucero, camino que va hacia los cultivos de aguacate, secundario al camino hacia las antenas, El Floripondio, faldas del Nevado de Colima, 11.VII. 2012, C. Torres-Preciado 511 (IBUG).

Hábitat: lignícola, solitario a gregario; bosque de Pinus-Quercus; 2500 m s.n.m.

Distribución: se amplía su distribución al citarse para Jalisco, ya que solo se conocía para el estado de Veracruz (Gándara-Zamorano y Ramírez-Cruz, 2005).

Notas taxonómicas: una especie similar es Hohenbuehelia petaloides (Bull.) Schulzer, pero se diferencia principalmente por presentar un píleo tomentoso, de color café-amarillento (Gándara-Zamorano y Ramírez-Cruz, 2005). Microscópicamente se observan esporas elipsoides a ampliamente elipsoides (5-)5.5-7.5(-8) × (3-)3.5-4.5(-5) $\mu \mathrm{m}, \mathrm{Q}=1.2-1.66$. Ambas especies presentan metuloides con cristales en el ápice.

\section{Psathyrellaceae}

- Psathyrella longistriata (Murrill) A.H. Sm., Mushrooms in their natural habitats: 552. 1949. TIPO: ESTADOS UNIDOS DE AMÉRICA. Washington: Seattle, sobre tierra y restos de madera, 20.X.1911, W. A. Murrill 233 (WTU).

Fig. 3 (D, E y F).

इStropharia longistriata Murrill, Mycologia 4(6): 301. 1912.

Píleo 20-40 mm de diámetro, convexo-aplanado, superficie glabra, margen estriado, color café a cafégrisáceo; láminas adheridas, muy juntas, subanchas, borde entero, color café oscuro o café chocolate; estípite 60-90 × 1-3 mm, central, más o menos uniforme, superficie fibrilosa, hueco, blanquecino, con anillo apical, membranoso, estriado, color blanco con borde más oscuro; esporas 8-10.4 × 4-4.4 $\mu \mathrm{m}, \mathrm{Q}=1.7-2$, elongadas, lisas, poro germinal hialino, pared gruesa $(\geq 0.5 \mu \mathrm{m})$, color verde-olivo, café-verdoso; pleurocistidios 37.6-50.4 × 9.6-14.4 $\mu \mathrm{m}$, subutriformes a claviformes, algunos constricción central, ápice subcapitado a obtuso, fíbula basal, hialinos; pileipellis 
un epitelio, 20.8-36.8 × 16.8-32 $\mu \mathrm{m}$, de células claviformes, subglobosas, color anaranjado-amarillento.

Material estudiado: MÉXICO. Jalisco, municipio Zapotlán el Grande, Puerto El Floripondio, 20 minutos al suroeste de Ciudad Guzmán, 7.VII.1984, G. Nieves 276 (IBUG).

Hábitat: lignícola, sobre madera en descomposición, gregario; bosque de Pinus-Quercus; sin datos de altitud. La especie en estudio ha sido citada de hábitat humícola o creciendo sobre restos de madera (Desjardin et al., 2015).

Distribución: se conoce su distribución en Estados Unidos de América y Australia (Smith, 1972, 1975; Desjardin et al., 2015). Esta especie se registra por primera vez para México.

Notas taxonómicas: Psathyrella longistriata se distingue por el píleo glabro con escasas fibrillas y margen estriado, con estípite de color blanquecino y la presencia de un anillo bien definido, estriado en la superficie. El material examinado concuerda con lo descrito por Smith (1972, 1975) y Desjardin et al. (2015). Una especie afín a Psathyrella longistriata es P. ellenae A.H. Sm.; sin embargo, esta última no presenta un anillo estriado. Otra especie parecida es P. barrowsii A.H. Sm. pero se diferencia al carecer de fibrillas en el píleo, estrías en el margen y en el anillo (Desjardin et al., 2015).

\section{Strophariaceae}

** Pholiota lubrica (Pers.) Singer, Lilloa 22: 516. 1951. TIPO: Espécimen tipo no designado, ni se menciona en cual herbario se depositó.

$\equiv$ Agaricus lubricus Pers., Synopsis methodica fungorum 2: 307. 1801.

三 Flammula lubrica (Pers.) P. Kumm., Der Führer in die Pilzkunde: 81. 1871.

ミInocybe lubrica (Pers.) Roze, Bulletin de la Société Botanique de France 23: 113. 1876.

इ Dryophila lubrica (Pers.) Quél., Enchiridion Fungorum in Europa media et praesertim in Gallia Vigentium: 70. 1886.

三 Flammopsis lubrica (Pers.) Fayod, Annales des Sciences Naturelles Botanique ser. 7, 9: 356. 1889.

इ Gymnopilus lubricus (Pers.) S. Imai, Journal of the Faculty of Agriculture of the Hokkaido Imperial University 43: 231. 1938.

इ Pholiotina lubrica (Pers.) Singer, Lilloa 22: 516. 1951.

= Agaricus decussatus Fr., Epicrisis Systematis Mycologici: 185. 1874.

= Agaricus decussatus subsp. illustris Kalchbr., Icones selectae Hymenomycetum Hungariae 2: 26, t. 25: 1. 1874

= Pholiota lenta var. medullata Wichanský, Mykologicky Sbornik: 144. 1967.

Píleo $1.5 \mathrm{~cm}$ de diámetro, convexo, recto, superficie viscosa-pruinosa, margen entero, color anaranjado amarillento con tonos anaranjado-rojizo más obscuro hacia el centro; láminas adheridas, subdistantes, angostas, borde crenado, color amarillo; estípite $5 \times 1-2 \mathrm{~cm}$, central, uniforme, rígido, color blanco con tonos café-amarillentos; esporas 6-8(-8.5) × 3.5-4.4(4.5) $\mu \mathrm{m}, \mathrm{Q}=(1.5-) 1.55-2(-2.12)$, elipsoides, en su mayoría elongadas a cilíndricas, apículo muy conspicuo, ápice truncado con poro germinal, pared gruesa $(\geq 0.5 \mu \mathrm{m})$, contenido granuloso refringente, inamiloides, gutuladas, color café-amarillento; basidios (21-)21.8-25.01(-25.1) × (5.7-)6-7(-7.4) $\mu \mathrm{m}$, claviformes, tetraspóricos, abundantes, contenido refringente, hialinos; pleurocistidios (50-)58-71(-74) × (11-)12-19(-20) $\mu \mathrm{m}$, ventricosos, ápice capitado, pared gruesa $(\geq 0.5 \mu \mathrm{m})$, fíbula basal, abundantes, hialinos; queilocistidios 50-65(68) $\times 11-17 \mu \mathrm{m}$, ventricosos, ápice capitado, similares a los pleurocistidios, pared gruesa $(\geq 1 \mu \mathrm{m})$, fíbula basal, abundantes, hialinos; pileipellis un ixocutis, hifas 5.1-12.5 $\mu \mathrm{m}$, pigmento incrustado conspicuo, pared gruesa $(\geq 0.5$ $\mu \mathrm{m})$, color café-amarillento.

Material estudiado: MÉXICO. Jalisco, municipio de Zapotlán el Grande, 8 km después del crucero, El Floripondio, camino a Las Víboras, antenas R.M.O., El Pajarito, faldas del Nevado de Colima, 26.IX.2011, C. TorresPreciado 405 (IBUG). 
Hábitat: terrícola, gregario; bosque de Pinus-Quercus con elementos de Abies; 2739 m s.n.m.

Distribución: Pholliota lubrica es citada de Europa y Estados Unidos de América (Breitenbach y Kränzlin, 1995; Noordeloos, 1999). Su distribución en México hasta ahora fue registrada del Estado de México (Guzmán, 1970; Valenzuela et al., 2004).

Notas taxonómicas: de acuerdo con Holec (2001), una característica de importancia taxonómica dentro del género Pholiota es la presencia de cistidios para dividirlo en subgéneros. Pholiota lubrica se caracteriza por la ausencia de crisocistidios según lo señalado por Breitenbach y Kranzlin (1995), quienes mencionan la presencia de hifas pigmentadas con incrustaciones muy conspicuas, así como los cistidios con ornamentaciones en suápice, características que coinciden con lo observado en el material estudiado. Conforme con Noordeloos (1999), Pholliota lenta (Pers.: Fr.) Singer es parecida microscópicamente por la forma de esporas; sin embargo, la coloración de su basidioma es de tonos grisáceo-ocre a oscuros.

\section{Auriculariales}

\section{Exidiaceae}

- Ductifera exidioidea Lowy, J. Elisha Mitchell Scient. Soc. 80: 65. 1964. TIPO: GUATEMALA. Finca San Mayor, km 33 al oeste de la ciudad de Guatemala, sobre ramas caídas de Quercus sp., 25.VI.1963, G195 (LSUM). Fig. 3 $(A, B, C)$.

Hongo gelatinoso de forma foliosa-petaloide, superficie pegajosa provista con numerosos gránulos negros, color blanquecino a marrón; esporas 18.7-21.7(22.6) $\times$ 4.4-5.7 $\mu \mathrm{m}, \mathrm{Q}=3.8-4.58(-4.6)$, alantoides, ápice truncado, gutuladas, contenido refringente, hialinas; basidios 15.1-20.5(-21) × 8.8-11.8 $\mu \mathrm{m}$, tetraspóricos, con fragmobasidios de tipo ovoide, hialinos; gleocistidios 39.6$84 \times 4-9.1 \mu \mathrm{m}$, claviformes, ápice obtuso, muy abundantes, hialinos a café amarillento.
Material examinado: MÉXICO. Jalisco, municipio Zapotlán el Grande, 1 km después del crucero, 2 km después del camino que va hacia las antenas, El Floripondio, faldas del Nevado de Colima, 27.VI.2011, C. Torres-Preciado 471 (IBUG).

Hábitat: lignícola, gregario sobre una rama de Quercus L. en bosque de Pinus-Quercus; 2400 m s.n.m.

Distribución: la especie solo se ha citado de Guatemala (Lowy, 1964; Flores-Arzú et al., 2012) e India (Mahamulkar et al., 2002).

Notas taxonómicas:Ductifera exidioidea se caracteriza por sus esporas alantoides y gleocistidios hialinos, con muy escaso o sin contenido granular. El material estudiado concuerda con lo descrito por Lowy (1964), excepto por el tamaño de las esporas las cuales son más pequeñas, 15-18 $\times$ 4.5-5.5 $\mu \mathrm{m}$ con respecto a nuestro ejemplar. Una especie afín es Ductifera sucina (Möller) K. Wells; sin embargo, esta se diferencia por la textura suave, cerosa-gelatinosa y color del basidioma café pálido, tamaño de esporas (9.6.-12.8 $\times$ 4-6.4 $\mu \mathrm{m}$ ) y forma de basidios oblongo-claviformes (Lowy, 1964).

Boletales

\section{Sclerodermataceae}

** Scleroderma aff. pseudostipitatum Petch, Ann. R. bot. Gdns Peradeniya 7(1): 76. 1919. TIPO: SRI LANKA Hakgalla, IX.1908, T. Petch, sobre suelo (K).

इ Veligaster pseudostipitatus (Petch) Guzmán \& Tapia, Documents Mycologiques 25(98-100): 191. 1995.

Basidioma 150-250 × 120-220 mm, globoso, estipitado $10-30 \times 5-10(-20) \mathrm{mm}$, bien formado y con cordones miceliares blanquecinos; peridio delgado, $1 \mathrm{~mm}$ de grosor, rubescente, escamas planas con apariencia de verrugas, color café a café-rojizo obscuro; gleba color café-grisáceo cuando maduro; esporas (8-)10.4-12.8 $\mu \mathrm{m}$, 
no incluye ornamentación, $Q=1$, globosas, equinuladas, espinas cónicas 1.6-2.4 $\mu \mathrm{m}$ de altura, algunas curveadas, color verde-olivo, amarillentas.

Material estudiado: MÉXICO. Jalisco, municipio de Zapotlán el Grande, El Floripondio, Nevado de Colima, 9.IX.1998, G. Guzmán 32654 (IBUG, XAL).

Hábitat: terrícola, gregario; bosque de PinusQuercus; sin datos de altitud.

Distribución: la especie hasta ahora fue registrada de Sri Lanka y México, de este último citada del estado de Veracruz (Guzmán et al., 2013).

Notas taxonómicas: el material estudiado concuerda con lo observado por Guzmán et al. (2013), quien describe a Scleroderma spseudostipitatum con un basidioma estipitado con esporas (8.3-)10-14(-15) $\mu \mathrm{m}$, equinuladas con espinas $1-2(-2.5) \mu \mathrm{m}$, tamaño más grande que lo observado en el ejemplar aquí registrado. Una especie afín es S. verrucosum (Bull.) Pers. con esporas de (8-)9-12(-14) $\mu \mathrm{m}$, pero con estípite poco desarrollado y S. nitidum Berk. de (6-)7-11(-13) $\mu \mathrm{m}$, ambas especies con espinas más pequeñas a lo observado. Por su parte, Rifai (2017) diferencia a S. spseudostipitatum de S. nitidum por el diámetro más pequeño de sus esporas (6.6-9 $\mu \mathrm{m})$, espinas cónicas de hasta $1.8 \mu \mathrm{m}$ y el peridio con escamas café-rojizas. De acuerdo con la literatura consultada, no es clara la delimitación de $S$. pseudostipitatum y sus especies similares, por lo que el material aquí revisado se identifica como afín a esta especie, en espera que futuros estudios moleculares puedan elucidar los complejos de especies de este género.

\section{Discusión}

El número de taxones determinados, como resultado del presente trabajo, se incrementó de 140 a 408, de las cuales $30 \%$ se consideran potencialmente de importancia económica y ecológica por su valor comestible y/o micorrizógeno.

Se detectó que algunas especies típicas de cierto tipo de bosque están también presentes en otro, como es el caso de Laccaria laccata registrada del BP y encontrándose al igual en el BPE y BMM; situación similar a lo observado por Fierros et al. (2000) y en el área de estudio. Esto puede deberse, según lo mencionado por Fierros et al. (2000), a que los lugares muestreados tienen características peculiares donde la comunidad florística es muy variable y en la que puede asociarse al BPE, donde algunos elementos de ambos llegan a traslaparse, ocasionando la existencia de especies compartidas, situación observada en este estudio. Lo anterior descrito, se observa en especies micorrizógenas como Amanita caesarea (Scop.) Pers., la cual puede asociarse a especies de pino o encino. Asimismo, especies como Lentinus crinitus y Stereum ostrea, por citar algunos ejemplos, se tienen reportadas tanto de bosques templados como de tropicales (Frutis-Molina y Pinzón-Picaño, 1997; Esqueda-Valle et al., 1999; Chanona-Gómez et al., 2007; Villarruel-Ordaz et al., 2015).

La cita de 183 registros nuevos para el CVC es el resultado del estudio, en su mayoría, de las colecciones obtenidas en los últimos diez años, así como de la revisión o corroboración del material depositado en la colección micológica, lo que nos muestra a su vez lo mucho que falta por trabajar.

El listado de los 408 taxones registrados puede considerarse representativo, dado que supera en mucho lo encontrado en catálogos previos, no solo para la zona del CVC, sino también de otras contribuciones micobióticas realizadas en Colima y Jalisco (LópezRamírez y Medel, 2016; Padilla-Velarde et al., 2016; Terríquez Villanueva et al., 2017; Rodríguez et al., 2018, 2019). Es importante mencionar que se observaron localidades o municipios poco o nada explorados como es el caso de Zapotiltic, del que no se contaba con ningún registro, y que ahora aporta 10 este trabajo. Sin embargo, se considera necesario continuar con las exploraciones micológicas que enriquezcan el catálogo de hongos del área de estudio.

Asimismo, un mayor conocimiento de la micobiota del Complejo Volcán Nevado de Colima podría permitir evaluar su diversidad fúngica, distribución actual, y establecer estrategias de un posible aprovechamiento, manejo y conservación en esta zona reconocida por su alto ende- 
mismo en flora y fauna (Segura-Trujillo y Navarro Pérez, 2010).

Con base en los resultados obtenidos de las especies fúngicas determinadas, se muestra la importancia de este recurso para su posible aprovechamiento como fuente alimenticia local, y su manejo forestal en esta región que comprende el CVC, ya que desafortunadamente no son consumidos o aprovechados por los lugareños de estas localidades. Por lo anterior, se considera necesario conocer sobre su micobiota, más aún en este tipo de zonas en la que convergen tanto bosques templados como tropicales, siendo estos últimos tan escasamente explorados.

Otro aspecto importante para mencionar es sobre endemismo en hongos, de la que pocos estudios se tienen, que nos permitan conocer con exactitud la distribución tan amplia que muchas de las especies presentan. De acuerdo con la Norma Oficial Mexicana NOM-059-SEMARNAT-2020, se enlistan 46 especies de hongos bajo alguna categoría de riesgo, de las cuales nueve se presentan dentro del CVC, en donde ocho especies están bajo la categoría de amenazada (A) y una citada como sujeta a protección (Pr) (Cuadro 3). Asimismo, dentro de las categorías y criterios de la lista roja de la UICN (International Union for Conservation of Nature), de la que incluye 280 especies de hongos, solo una de ellas se tiene registrada del Complejo Volcánico Colima,
Boletinellus merulioides (Schwein.) Murrill, catalogada bajo el estatus de vulnerable (VU) (UICN, 2020).

\section{Contribución de autores}

CTP, OR y MJHF realizaron recolecta de material fúngico, su determinación y la corroboración de la identificación taxonómica de los ejemplares depositados previamente en la colección micológica del herbario IBUG. CTP y OR escribieron el manuscrito con el apoyo de MJHF y DFG. DFG colaboró en la elaboración de los cuadros y figuras, en la actualización de la base de datos, su análisis e interpretación, así como la validación de las especies fúngicas. Todos los autores contribuyeron a la discusión, revisión crítica del contenido y aprobación del manuscrito final.

\section{Financiamiento}

Este estudio fue apoyado por la Universidad de Guadalajara a través de los proyectos P3E (2015-2243 y 2016229388).

\section{Agradecimientos}

Los autores agradecen a Jacqueline Reynoso Dueñas por la revisión del manuscrito y sus valiosos comentarios. A Daniel Ulloa Olmos por la elaboración del mapa del área de estudio y el apoyo de Adrián Galván Corona, por el material fotográfico.

Cuadro 3: Especies de hongos incluidas en la NOM-059-SEMARNAT-2020 (SEMARNAT, 2020) presentes en el Complejo Volcánico de Colima, México. $\mathrm{A}=$ Amenazada; $\mathrm{Pr}=$ Sujeta a protección especial.

\begin{tabular}{lc}
\hline \multicolumn{1}{c}{ Especies } & Categoría \\
\hline Amanita muscaria (L.) Lam. & $\mathrm{A}$ \\
Cantharellus cibarius var. cibarius=C. cibarius Fr. & $\mathrm{Pr}$ \\
Gomphidius rutilus (Schaeff.) S. Lundell & $\mathrm{A}$ \\
Hygrophorus russula (Schaeff. ex Fr.) Kauffman & $\mathrm{A}$ \\
Morchella angusticeps Peck & $\mathrm{A}$ \\
Morchella conica=M. esculenta (L.) Pers. & $\mathrm{A}$ \\
Morchella costata Pers. & $\mathrm{A}$ \\
Morchella esculenta var. umbrina=M. esculenta (L.) Pers. & $\mathrm{A}$ \\
Psilocybe mexicana R. Heim & $\mathrm{A}$ \\
Psilocybe zapotecorum R. Heim & $\mathrm{A}$ \\
\hline
\end{tabular}




\section{Literatura citada}

Arceo-Orozco, A. 2011. Hongos macroscópicos de Bosque de Los Colomos, Guadalajara, Jalisco. Tesis de licenciatura. Universidad de Guadalajara. Guadalajara, Jalisco, México. 48 pp.

Arnolds, E. 1990. Tribus Hygrocybeae. In: Bas, C., T. H. Kuyper, M. E. Noordeloos y E. C. Vellinga (eds.). Flora agaricina Neerlandica, Vol. 2. Critical monographs on families of agarics and boleti occurring in the Netherlands. Balkema. Rhotterdam, The Netherlands. Pp. 71-115.

Arroyo, K. 2001. Macromicetos de la Barranca del Río Santiago en Zapopan, Jalisco, con énfasis en su conservación. Tesis de licenciatura. Universidad Autónoma de Guadalajara. Guadalajara, Jalisco, México. 51 pp.

Bandala, V. y L. Montoya. 2008. Type studies in the genus Crepidotus. Mycotaxon 103: 235-254.

Bautista, N., S. Chacón y G. Guzmán. 1986. Ascomycetes poco conocidos en México, III. Especies del estado de Morelos. Revista Mexicana de Micología 2: 85-104.

Bautista-González, J. A. y A. Moreno-Fuentes. 2014. Los hongos medicinales de México. In: Moreno Fuentes, A. y R. Garibay Orijel (eds.). La Etnomicología en México, estado del arte. Consejo Nacional de Ciencia y Tecnología (CONACYT), Universidad Autónoma del Estado de Hidalgo (UAEH), Universidad Nacional Autónoma de México (UNAM). Cd. Mx., México. Pp. 91-109.

Bautista-Hernández, S., T. Herrera, E. Aguirre-Acosta y M. Esqueda. 2011. Contribution to the taxonomy of Bovista in Mexico. Mycotaxon 118: 27-46. DOI: https://doi. org/10.5248/118.27

Beug, M. W., A. E. Bessette y A. R. Bessette. 2014. Ascomycete fungi of North America, a mushroom reference guide. University of Texas Press. Austin, USA. 502 pp.

Breitenbach, J. y F. Kränzlin. 1984. Fungi of Switzerland, Vol. 1. Ascomycetes. Verlang Mykologia. Lucerna, Switzerland. 310 pp.

Breitenbach, J. y F. Kränzlin. 1986. Fungi of Switzerland, Vol. 2. Non gilled fungi, Heterobasidiomycetes, Aphyllophorales, Gasteromycetes. Verlang Mykologia. Lucerna, Switzerland. $412 \mathrm{pp}$.

Breitenbach, J. y F. Kränzlin. 1991. Fungi of Switzerland, Vol. 3. Boletes and Agarics 1st part, Strobilomycetaceae and Boletaceae, Paxilaceae, Gomphidiaceae, Hygrophoraceae,
Tricholomataceae, Polyporaceae (lamellate). Verlang Mykologia. Lucerna, Switzerland. 361 pp.

Breitenbach, J. y F. Kränzlin. 1995. Fungi of Switzerland, Vol. 4. Agarics 2nd part, Entolomataceae, Pluteaceae, Amanitaceae, Agaricaceae, Coprinaceae, Bolbitaceae, Strophariaceae. Verlang Mykologia. Lucerna, Switzerland. $368 \mathrm{pp}$.

Breitenbach, J. y F. Kränzlin. 2000. Fungi of Switzerland, Vol. 5. Agarics 3rd part, Cortinariaceae. Verlang Mykologia. Lucerna, Switzerland. 338 pp.

Calonge, F. D. 1998. Gasteromycetes I. Lycoperdales, Nidulariales, Phallales, Sclerodermatales, Tulostomatales. Flora Micológica Cramer. Madrid, España. 271 pp.

Camacho-Sánchez, M. 2010. Estudio taxonómico del complejo de Pleurotus, Lentinus y Panus en México. Tesis de maestría. Instituto de Ecología, A.C. Xalapa, Veracruz, México. 130 pp.

Carl Zeiss Microscopy. 2020. AxioVision 4 Module Interactive Measurement. Nueva York, USA. https://www.microshop.zeiss. com/en/us/system/axiovision+software/software+axiovision/ biomed\%252Fmat+modules/000000-1235-871\# (consultado mayo de 2020).

Ceballos, G., P. Rodríguez y R. Medellín. 1998. Assessing conservation priorities in megadiverse Mexico: mammalian diversity, endemicity, and endangerment. Ecological Applications 8(1): 8-17. DOI: https://doi.org/10.1890/10510761(1998)008[0008:ACPIMM]2.0.CO;2

Chacón, S. y G. Guzmán. 1983. Ascomycetos pocos conocidos en México. Boletín de la Sociedad Mexicana de Micología 18: 183-218.

Chanona-Gómez, F., R. H. Andrade-Gallegos, J. Castellanos-Albores y J. E. Sánchez. 2007. Macromicetos del Parque Educativo Laguna Bélgica, municipio de Ocozocoautla de Espinoza, Chiapas, México. Revista Mexicana de Biodiversidad 78(2): 369-381.

Cibrián-Tovar, D., D. Alvarado-Rosales y S. E. García-Díaz. 2007. Enfermedades Forestales de México/Forest Diseases in Mexico. Universidad Autónoma de Chapingo; Comisión Nacional Forestal (CONAFOR), Secretaría de Medio Ambiente y Recursos Naturales (SEMARNAT), México; Forest Service United States Department of Agriculture (USDA), EUA; Natural Resources Canada (NRCAN) Forest Service, Canadá y Comisión Forestal para América del Norte (CFAN), Organización de las Naciones Unidas para 
la Alimentación y la Agricultura (FAO). Chapingo, México. $587 \mathrm{pp}$.

Cifuentes, J., M. Villegas, L. Pérez-Ramírez, M. Bulnes, V. Corona, M. del R. González, I. Jiménez, A. Pompa y G. Vargas. 1990. Observaciones sobre la distribución, hábitat e importancia de los hongos de los Azufres, Michoacán. Revista Mexicana de Micología 6: 11-28.

Cox, C. B. 2001. The biogeographic regions reconsidered. Journal of Biogeography 28(4): 511-523. DOI: https://doi. org/10.1046/j.1365-2699.2001.00566.x

Dennis, R. W. G. 1981. British Ascomycetes. Lubrecht \& Cramer Ltd. Vaduz, Liechtenstein. 585 pp.

Desjardin, D. E., M. G. Wood y F. A. Stevens. 2015. California Mushrooms: The Comprehensive Identification Guide. Timer Press. Portland, USA. 560 pp.

Díaz-Barriga, H., F. Guevara-Fefer y R. Valenzuela. 1988. Contribución al conocimiento de los macromicetos del estado de Michoacán. Acta Botanica Mexicana 2: 21-44. DOI: https://doi.org/10.21829/abm2.1988.564

Espinosa, D. J., J. Morrone, C. Aguilar y J. Llorente. 2000. Regionalización biogeográfica de México: provincias bióticas. In: Llorente J., E. Gonzáles y N. Papayero (eds.). Biodiversidad, taxonomía y biogeografía de artrópodos de México, hacia una síntesis de su conocimiento. Comisión Nacional para el Conocimiento y Uso de la Biodiversidad (CONABIO). Cd. Mx., México. Pp. 61-94.

Esqueda-Valle, M., E. Pérez-Silva, T. Herrera, F. San Martín y R. Santos-Guzmán. 1999. Macromicetos de Selva Baja Caducifolia. Álamos, Sonora, México. Revista Mexicana de Micología 15: 73-78.

Esqueda-Valle, M., A. Sánchez, M. Rivera, M. L. Coronado, M. Lizárraga y R. Valenzuela. 2009. Primeros registros de hongos gasteroides en la Reserva Forestal Nacional y Refugio de Fauna Silvestre Ajos-Bavispe, Sonora, México. Revista Mexicana de Micología 30: 19-29.

Esqueda-Valle, M., A. Sánchez, M. L. Coronado, A. Gutiérrez, M. Lizárraga y R. Valenzuela. 2011. Nuevos registros de hongos gasteroides en la Reserva de Biosfera Sierra de Álamos-Río Cuchujaqui. Revista Mexicana de Micología 34: 43-51.

Fa, J. E. y L. M. Morales. 1993. Patterns of mammalian diversity in Mexico. In: Ramamoorthy, T. P., R. Bye, A. Lot y J. E. Fa (eds.). Biological diversity of Mexico: Origins and distribution. Oxford University Press. Nueva York, USA. Pp. 319-361.
Fernández-Sasia, R. 2013. Tres Inocybe de la sección Rimosae. Boletín Informativo de la Sociedad Micológica Extremeña 13: 28-34.

Fierros, M. L. y L. Guzmán-Dávalos. 1995. Inventario preliminar de los hongos macroscópicos de Sierra de Quila, Jalisco, México. Boletín IBUG (Instituto de Botánica, Universidad de Guadalajara), Época 3, 3(1-3): 129-142.

Fierros, M. L., J. L. Navarrete-Heredia y L. Guzmán-Dávalos. 2000. Hongos macroscópicos de Sierra de Quila, Jalisco, México: diversidad y similitud fungística. Revista de Biología Tropical 48(4): 931-937.

Flores-Arzú, R., O. Comandini y A. C. Rinaldi. 2012. A preliminary checklist of macrofungi of Guatemala, with notes on edibility and traditional knowledge. Mycosphere 3(1): 1-21. DOI: https://doi.org/10.5943/mycosphere/3/1/1

Flores-Villela, O. 1993. Herpetofauna of Mexico: Distribution and endemism. In: Ramamoorthy, T. P., R. Bye, A. Lot y J. E. Fa (eds.). Biological diversity of Mexico: Origins and distribution. Oxford University Press. Nueva York, USA. Pp. 253-280.

Frutis, I., R. E. Chio y A. Estrada-Torres. 1985. Nuevos registros de macromicetos del Estado de México. Revista Mexicana de Micología 1: 285-300.

Frutis-Molina, I. y L. M. Pinzón-Picaño. 1997. Macromicetos. In: Gozález Sorianoo, E., R. Dirzo y R. C. Vogt (eds.). Historia Natural de los Tuxtlas. Universidad Nacional Autónoma de México (UNAM) y Comisión Nacional para el Conocimiento y Uso de la Biodiversidad (CONABIO). Cd. Mx., México. Pp. 201-210.

Gándara-Zamorano, E. y V. Ramírez-Cruz. 2005. El género Hohenbuehelia (Basidiomycotina, Agaricales, Tricholomataceae) en Veracruz, México. Revista Mexicana de Micología 21: 29-37.

Gándara-Zamorano, E., L. Guzmán-Dávalos, G. Guzmán y O. Rodríguez. 2014. Inventario micobiótico de la región de Tapalpa, Jalisco, México. Acta Botanica Mexicana 107: 165187. DOI: https://doi.org/10.21829/abm107.2014.207

García-Jiménez, J. 1999. Estudio sobre la taxonomía, ecología y distribución de algunos hongos de la familia Boletaceae (Basidiomycetes, Agaricales) de México. Tesis de maestría. Universidad Autónoma de Nuevo León. Linares, Nuevo León, México. 334 pp.

García-Jiménez, J. y J. Castillo. 1981. Las especies de Boletaceos 
y Gomfidaceos conocidos en Nuevo León. Boletín de la Sociedad Mexicana de Micología 15: 158-164.

Garibay-Orijel, R. y F. Ruan-Soto. 2014. Listado de los hongos silvestres consumidos como alimento tradicional en México. In: Moreno Fuentes, A. y R. Garibay Orijel (eds.). La Etnomicología en México, estado del arte. Consejo Nacional de Ciencia y Tecnología (CONACYT), Universidad Autónoma del Estado de Hidalgo (UAEH), Universidad Nacional Autónoma de México (UNAM). Cd. Mx., México. Pp. 91-109.

Gilberson, R. L. y L. Ryvarden. 1986. North American Polypores, Vol. 1. Abortiporus-Lindtneria. Fungiflora A/S. Oslo, Norway. $433 \mathrm{pp}$.

Gilberson, R. L. y L. Ryvarden. 1987. North American Polypores, Vol. 2. Megasporoporia-Wrightoporia. Fungiflora A/S. Oslo, Norway. 885 pp.

Gómez-Reyes, V. M., O. Tinoco Marina, A. Terrón-Alfonso, M. Gómez-Peralta, C. A. Tena Morelos y F. Garza Ocañas. 2014. Efecto de los incendios forestales en la riqueza y composición de macromicetos. Revista Mexicana de Micología 39: 21-30.

Groposo, C., C. Loguercio-Leite y A. Góes-Neto. 2007. Fuscoporia (Basidiomycota, Hymenochaetales) in Southern Brazil. Mycotaxon 101: 55-63.

Guzmán, G. 1970. Notas sobre el género Pholliota en México. Boletín de la Sociedad Mexicana de Micología 4: 25-27.

Guzmán, G. 1977. Identificación de los hongos, comestibles, venenosos, alucinantes y destructores de madera. Editorial Limusa. Cd. Mx., México. 452 pp.

Guzmán, G. 1994. Los hongos en la medicina tradicional de Mesoamérica y de México. Revista Iberoamericana de Micología 11: 81-85.

Guzmán, G. 1998. Las especies de Psilocybe (Fungi, Basidiomycotina, Agaricales) conocidas de Jalisco (México) y descripción de dos nuevas especies. Acta Botanica Mexicana 43: 23-32. DOI: https://doi.org/10.21829/ abm43.1998.800

Guzmán, G. 2004. Los hongos de El Edén, Quintana Roo (Introducción a la micobiota tropical de México). Instituto de Ecología, A.C. y Comisión Nacional para el Conocimiento y Uso de la Biodiversidad. Xalapa, México. 316 pp.

Guzmán, G. 2008. Diversity and use of traditional Mexican medicinal fungi. A review. International Journal of Medicinal
Mushrooms 10(3): 209-217. DOI: https://doi.org/10.1615/ IntJMedMushr.v10.i3.20

Guzmán, G., A. Cortes-Pérez, L. Guzmán-Dávalos, F. RamírezGuillén y M. R. Sánchez-Jácome. 2013. An emendation of Scleroderma, new records, and review of the known species in Mexico. Revista Mexicana de Biodiversidad 84: S173-191. DOI: https://doi.org/10.7550/rmb.31979

Guzmán-Dávalos, L. 1996. New records of the genus Gymnopilus (Agaricales, Cortinariaceae) from Mexico. Mycotaxon 59: 61-78.

Guzmán-Dávalos, L. 2003. Actualización de la base de datos de hongos macroscópicos de Jalisco. Universidad de Guadalajara, Centro Universitario de Ciencias Biológicas y Agropecuarias, Bases de datos SNIB2010-Comisión Nacional para el Conocimiento y Uso de la Biodiversidad, proyectos No. U013 y G013. Cd. Mx., México.

Guzmán-Dávalos, L. y G. Guzmán. 1979. Estudio ecológico comparativo entre los hongos (macromicetos) de los bosques tropicales y los de coníferas del sureste de México. Boletín de la Sociedad Mexicana de Micología 13: 89-125.

Guzmán-Dávalos, L. y G. Guzmán. 1995. Toward a monograph of the genus Gymnopilus (Cortinariaceae) in Mexico. Documents Mycologiques 25(98-100): 197-212.

Hall, I. R., S. L. Stephenson, P. K. Buchanan, W. Yun y A. L. J. Cole. 2003. Edible and poisonous Mushrooms of the world. Timber Press Inc. Christchurch, New Zeland. 372 pp.

Hansen, L. y H. Knudsen. 2000. Nordic Macromycetes, Vol. 1. Nordsvamp. Copenhagen, Denmark. 309 pp.

He, Mao-Qiang, Z. Rui-Lin, K. D. Hyde, D. Begerow, M. Kemler, A. Yurkov, E. H. C. McKenzie, O. Raspé, M. Kakishima, S. Sánchez-Ramírez, E. C. Vellinga, R. Halling, V. Papp, I. V. Zmitrovich, B. Buyck, D. Ertz, N. N. Wijayawardene, C. BaoKai, N. Schoutteten, Xinn-Zhan Liu, Tai-Hui Li, Yi-Jian Yao, Xin-Yu Zhu, An-Qi Liu, Guio-Jie Li, Ming-Zhe Zhang, Zhi-Lin Ling, B. Cao, V. Antonín, T. Boekhout, B. D. Barbosa de Silva, E. De Crop, Cony-Decock, B. Dima, A. Kumar Dutta, J. W. Fell, J. Geml, M. Ghobad-Nejhad, A. J. Giachini, T. B. Gilbertoni, S. P. Gorjón, D. Haelewaters, Shuang-Hui He, B. P. Hodkinson, E. Horak, T. Hoshino, A. Justo, Youn-Woon Lim, N. Menolli Jr., A. Mešić, Jean-Marc Moncalvo, G. M. Mueller, L. G. Nagy, R. Henrik Nilsson, M. Noordeloos, J. Nuytinck, T. Orihara, C. Rattchadawan, M. Rajchenberg, A. G. S. Silva-Filho, M. Aloisio Sulzbacher, Z. Tkalčec, R. Valenzuela, A. Verbeken, 
A. Vizzini, F. Wartchow, Tie-Zheng Wei, M. Weib, ChangLin Zhao y P. M. Kirk. 2019. Notes, outline and divergence times of Basidiomycota. Fungal Diversity 99: 105-367. DOI: https://doi.org/10.1007/s13225-019-00435-4

Herrera-Fonseca, M., L. Guzmán-Dávalos y O. Rodríguez. 2002. Contribución al conocimiento de la micobiota de la región de San Sebastián del Oeste, Jalisco, México. Acta Botanica Mexicana 58: 19-50. DOI: https://doi.org/10.21829/ abm58.2002.888

Hesler, L. R. 1969. North American species of Gymnopilus, Mycologia Memoirs 3. Hafner Publ Co. New York, USA. 117 pp.

Holec, J. 2001. The genus Pholiota in central and western Europe. Libri Botanici 20. Eching, Alemania. 220 pp.

Index Fungorum. 2019. The global fungal nomenclator. http:// www.indexfungorum.org/names/Names.asp (consultado agosto de 2019).

INIFAP. 2019. Instituto Nacional de Investigación Forestal, Agrícola y Pecuarias. https://clima.inifap.gob.mx/Inmysr (consultado diciembre de 2019).

Janos, D. P. 1983. Tropical micorrizas, nutrient cycles and plant growth. In: Sutton, S. L., T. C. Whitmore y A. C. Chadwick (eds.). Tropical rain forest: ecology and management. Blackwell Scientific Publishers. Oxford, USA. Pp. 327-345.

Jardel, E. J., R. Cuevas, A. L. Santiago y J. M. Rodríguez. 2014. Ecología y manejo de los bosques mesófilos de montaña. In: Gual, M. y A. Rendón-Correa (eds.). El bosque mesófilo de montaña en México. Comisión Nacional para el Conocimiento y Uso de la Biodiversidad (CONABIO). Cd. Mx., México. Pp. 141-188.

Kränzlin, F. 2005. Fungi of Switzerland, Vol. 6. Russulaceae. Lactarius, Russula. Verlang Mykologia. Lucerna, Switzerland. $310 \mathrm{pp}$.

Kuyper, Th. W. 1986. A revision of the genus Inocybe in Europe, I. Subgenus Inosperma and the smooth-spored species of subgenus Inocybe. Peersonia Supplement 3: 1-247.

Landeros, F. y L. Guzmán-Dávalos. 2013. Revisión del género Helvella (Ascomycota: Fungi) en México. Revista Mexicana de Biodiversidad 84: S3-S20. DOI: https://dx.doi. org/10.7550/rmb.31608

Largent, D. L., D. Johnson y R. Watling. 1977. How to identify mushrooms to genus III: Microscopic features. Mad River Press. Eureka, USA. 148 pp.
Larsson, E., M. Ryberg, P.-A. Moreau, Å. Decuse Mathiesen y S. Jacobsson. 2009. Taxonomy and evolutionary relationships within species of section Rimosae (Inocybe) based on ITS, LSU and mtSSU sequence data. Persoonia 23: 86-98. DOI: http://dx.doi.org/10.3767/003158509X475913

López-Ramírez, A. y R. Medel. 2016. Hongos (Fungi). In: Cruz Angón, A., M. A. Ortega Huerta, E. D. Melgarejo, H. Perdomo Velázquez y H. Perdomo Velázquez (eds.). La Biodiversidad en Colima. Estudio de Estado. Comisión Nacional para el Conocimiento y Uso de la Biodiversidad (CONABIO). Cd. Mx., México. Pp. 177-181.

Lowy, B. 1964. New species of Tremellales from Guatemala. Journal of the Elisha Mitchell Scientific Society 80(2): 65-70.

Magaña-Martínez, C. S. y J. G. Palacios-Vargas. 2010. Colémbolos (Hexapoda) del Nevado de Colima, Jalisco, México. Dugesiana 17(1): 73-80.

Mahamulkar, S. H., B. D. Kundalkar y M. S. Patil. 2002. Studies in fleshy and gelly fungi-Tremellales. Indian Phytopathology 55(4): 464-468.

Medel, R. 2002. Nuevos registros de Pyrenomycetes (Ascomycotina) en México. Boletín de la Sociedad Botánica de México 70: 7985. DOI: http://dx.doi.org/10.17129/botsci.1656

Medel, R., G. Guzmán y S. Chacón. 1999. Especies de macromicetos citadas de México IX. Ascomycetes, Parte III: 1983-1996. Acta Botanica Mexicana 46: 57-72. DOI: https:// doi.org/10.21829/abm46.1999.816

Montañez, D., M. E. Noordeloos, O. Rodríguez, O. Vargas y L. Guzmán-Dávalos. 2016. Notes on the genus Entoloma (Basidiomycota, Agaricales) in two volcanic areas of Jalisco, Mexico. Phytotaxa 277(3): 211-236. DOI: http://dx.doi. org/10.11646/phytotaxa.277.3.1

Murrill, W. A. 1911. The Agaricaceae of tropical North America, II. Mycologia 3: 79-91. DOI: https://doi.org/10.1080/0027551 4.1911.12017666

MycoBank. 2019. Fungal Databases, Nomenclature and species banks. http://www.mycobank.org/quicksearch.aspx (consultado agosto de 2019).

Nieves, G. 1985. Contribución al conocimiento de los macromicetos del Bosque La Primavera, Zapopan, Jalisco. Tesis de licenciatura. Universidad de Guadalajara. Guadalajara, Jalisco, México. 74 pp.

Noordeloos, M. E. 1999. Strophariaceae. In: Bas, C., T. H. Kuyper, M. E. Noordeloos y E. C. Vellinga (eds.). Flora agaricina 
Neerlandica, Vol. 4. Critical monographs on families of agarics and boleti occurring in the Netherlands. Balkema. Rhotterdam, Netherlands. Pp. 27-107.

Nuñez, M. y L. Ryvarden. 2001. East Asian polypores 2. Polyporaceae s. lato. Synopsis Fungorum 14: 170-522.

Oliva-León, M. A. 2000. Contribución al conocimiento de los hongos (macromicetos) de la laguna de Sayula, Jalisco. Tesis de licenciatura. Universidad de Guadalajara. Guadalajara, Jalisco, México. 43 pp.

Padilla-Velarde, E. E., G. Zarco-Velazco, L. Guzmán-Dávalos y R. Cuevas-Guzmán. 2016. Primera contribución al conocimiento de Macromicetos de la vertiente norte del cerro El Cípil, en la costa sur de Jalisco. Acta Botanica Mexicana 114: 137-167. DOI: https://doi.org/10.21829/ abm114.2016.1105

Pegler, D. N. 1977. A preliminary agaric flora of East Africa. Kew Bulletin Additional Series VI. Her Majesty's St. Office. London, UK. 615 pp.

Pegler, D. N. 1983. Agaric flora of the Lesser Antilles. Kew Bulletin Additional Series IX. Her Majesty's St. Office. London, UK. 668 pp.

Pegler, D. N. 1986. Agaric flora of Sri Lanka. Kew Bulletin Additional Series XII. Her Majesty's St. Office. London, UK. 519 pp.

Pegler, D. N., T. Laessoe y B. M. Spooner. 1995. British puffballs, earthstars and stinkhorns: an account of the British gasteroid fungi. Royal Botanic Gardens Kew. London, UK. 255 pp.

Pérez-Moreno, J., M. Martínez-Reyes, A. Yescas-Pérez, A. Delgado-Alvarado y B. Xoconostle-Cázares. 2008. Wild mushroom markets in central Mexico and a case study at Ozumba. Economic Botany 62: 425-436. DOI: https://doi. org/10.1007/s12231-008-9043-6

Pérez-Silva, E., M. Esqueda-Valle, T. Herrera y M. Coronado. 2006. Nuevos registros de Agaricales de Sonora, México. Revista Mexicana Biodiversidad 77(1): 23-33.

Pérez-Silva, E., T. Herrera y A. Ocampo-López. 2011. Nuevos registros de macromicetos para el Municipio de Temascaltepec, Estado de México. Revista Mexicana de Micología 34: 23-30.

Phillips, R. 1991. Mushroom of North America. Little, Brown Co. (P.). Boston, USA. 139 pp.

Phillips, R. 2006. Mushrooms. A comprehensive guide with over 1,250 detailed photographs of mushrooms and other fungi. Macmillan. London, UK. 388 pp.
Ramírez-Terrazo, A., A. Montoya-Esquivel y J. Caballero-Nieto. 2014. Una mirada al conocimiento tradicional sobre los hongos tóxicos en México. In: Moreno Fuentes, A. y R. Garibay Orijel (eds.). La Etnomicología en México, estado del arte. Consejo Nacional de Ciencia y Tecnología (CONACyT), Universidad Autónoma del Estado de Hidalgo (UAEH), Universidad Nacional Autónoma de México (UNAM). Cd. Mx., México. Pp. 113-141.

Raymundo, T., R. Díaz-Moreno, S. Bautista-Hernández, E. AguirreAcosta y R. Valenzuela. 2012. Diversidad de ascomicetes macroscópicos en Bosque Las Bayas, municipio de Pueblo Nuevo, Durango, México. Revista Mexicana de Biodiversidad 83(1): 1-14. DOI: https://dx.doi.org/10.22201/ ib.20078706e.2012.1.1241

Raymundo, T., R. Valenzuela, S. Bautista-Hernández, M. Esqueda, J. Cifuentes y L. Pacheco. 2013a. El género Fuscoporia (Hymenochaetales, Basidiomycota) en México. Revista Mexicana de Biodiversidad 84: S50-S69. DOI: https://doi. org/10.7550/rmb.31604

Raymundo, T., E. Aguirre-Acosta, S. Bautista-Hernández, M. Contreras-Pacheco, P. Garma, H. León-Avendaño y R. Valenzuela. 2013b. Catálogo de los Ascomycota en los bosques de Santa Martha Latuvi, Sierra Norte, Oaxaca, México. Boletín de la Sociedad Micológica de Madrid 37: 13-29.

Raymundo, T., R. Valenzuela, Y. García-Martínez, M. A. BravoÁlvarez, J. C. Ramírez-Martínez, S. Bautista-Hernández, M. Palacios-Pacheco e I. Luna-Vega. 2019. Ascomycetes (Fungi) from the relic forest of Fagus grandifolia subsp. mexicana in eastern Mexico. Phytotaxa 418(1): 001-041. DOI: https:// doi.org/10.11646/phytotaxa.418.1.1.

Rifai, M. A. 2017. Catatan Tentang Scleroderma pseudostipitatum Petch, Scleroderma verrucosum (Bull.) Pers., and Scleroderma nitidum Berk. (Gasteromycetes). Jurnal Mikologi Indonesia 1(1): 11-14. DOI: http://doi. org/10.46638/jmi.v1i1.9

Rodríguez, O. 2013. El género Pluteus (Agaricales, Pluteaceae) en México. Revista Mexicana de Biodiversidad 84: 128-151. DOI: https://doi.org/10.7550/rmb.31610

Rodríguez, O. y L. Guzmán-Dávalos. 2001. Clave dicotómica de las especies del género Pluteus Fr. (Pluteaceae) conocidas de la región de Nueva Galicia y algunas áreas aledañas, México. Acta Botanica Mexicana 57: 23-36. DOI: https:// doi.org/10.21829/abm57.2001.882 
Rodríguez, O., M. Garza y L. Guzmán-Dávalos. 1994. Inventario preliminar de los hongos del Volcán de Tequila, Estado de Jalisco, México. Revista Mexicana de Micología 10: 103-111.

Rodríguez, O., D. Figueroa-García y M. J. Herrera-Fonseca. 2018. Catálogo de los hongos del Volcán de Tequila, municipio de Tequila, Jalisco, México. Polibotánica 45: 15-33. DOI: https://doi.org/10.18387/polibotanica.45.3

Rodríguez, O., D. Figueroa-García y M. J. Herrera-Fonseca. 2019. Catálogo de los hongos de San Sebastián del Oeste, municipio de San Sebastián del Oeste, Jalisco, México. Acta Botanica Mexicana 126: e1364. DOI: https://doi. org/10.21829/abm126.2019.1364

Rodríguez, O., M. Herrera-Fonseca y A. Galván-Corona. 2013. Nuevos registros de Basidiomycota para Jalisco, México. Acta Botanica Mexicana 105: 45-58. DOI: https://doi. org/10.21829/abm105.2013.226

Rodríguez, O., A. Galván-Corona, A. R. Villalobos-Arámbula, G. Vargas y L. Guzmán-Dávalos. 2009. Pluteus horakianus, a new species from Mexico. Sydowia 61: 39-52.

Rodríguez, O., A. Galván-Corona, A. R. Villalobos-Arámbula, A. Rodríguez y L. Guzmán-Dávalos. 2010a. A new species of Pluteus (Pluteaceae, Agaricales) from Mexico. Mycotaxon 112: 163-172. DOI: https://doi.org/10.5248/112.163

Rodríguez, O., M. Herrera-Fonseca, M. R. Sánchez-Jácome, I. Álvarez, R. Valenzuela, J. García y L. Guzmán-Dávalos. 2010b. Catálogo de la Micobiota del bosque La Primavera, Jalisco. Revista Mexicana de Micología 32: 29-40.

Romero-Bautista, L., G. Pulido-Flores y R. Valenzuela. 2010. Estudio micoflorístico de los hongos poliporoides del estado de Hidalgo, México. Polibotánica 29: 1-28.

Ruiz Rodríguez, M. E. y L. M. Pinzón-Picaseño. 1994. Caracteres culturales de Fomitopsis pinicola y Heterobasidion annosum, hongos xilófagos de importancia forestal asociados a pudriciones en oyamel. Boletín de la Sociedad Botánica de México 54: 225-250. DOI: https://doi.org/10.17129/ botsci.1432

Rzedowski, J. 1978. Vegetación de México. Ed. Limusa. México, D.F., México. 432 pp.

Sánchez-García, M., J. Cifuentes-Blanco y P. Brandon Matheny. 2013. Revisión taxonómica del género Melanoleuca en México y descripción de especies nuevas. Revista Mexicana de Biodiversidad 84: S111-S127. DOI: https://doi. org/10.7550/rmb.31569
Sánchez-Jácome, M. R. y L. Guzmán-Dávalos. 1997. Nuevos registros de Thelephora (Aphyllophorales, Basidiomycotina) para México. Revista Mexicana de Micología 13: 70-77.

Sánchez-Jácome, M. R. y L. Guzmán-Dávalos. 2005. New records of Ascomycetes from Jalisco, Mexico. Mycotaxon 92: 177191.

Sánchez-Jácome, M. R. y L. Guzmán-Dávalos. 2011. Hongos citados para Jalisco, II. Ibugana 16: 25-60.

Santiago, G., J. Cifuentes y M. Villegas. 1984. Contribución al conocimiento del género Amanita, subgénero Amanita en México. Boletín de la Sociedad Mexicana de Micología 19(3): 93-105.

Santillán, R. E. y R. Valenzuela. 1986. La familia Hygrophoraceae en México, I. Especies no citadas anteriormente. Revista Mexicana de Micología 2: 207-216.

Seaver, F. J. 1910. The Hypocreales of North America: III. Mycologia 2(2): 48-92. DOI: https://doi.org/10.2307/3753520

Segura-Trujillo, C. A. y S. Navarro-Pérez. 2010. Escenario y problemática de conservación de los murciélagos (Chiroptera) cavernícolas del Complejo Volcánico de Colima, Jalisco-Colima, México. Therya 1(3): 189-206. DOI: https:// doi.org/10.12933/therya-10-23

SEMARNAT. 2020. Norma Oficial Mexicana NOM-059-SEMARNAT-2020. Protección ambiental-Especies nativas de México de flora y fauna silvestres-Categorías de riesgo y especificaciones para su inclusión, exclusión o cambioLista de especies en riesgo. Secretaría del Medio Ambiente y Recursos Naturales. Diario Oficial de la Federación. Cd. Mx., México. http://dof.gob.mx/nota_detalle. php?codigo $=5424575 \&$ fecha $=05 / 02 / 2016$

SEMARNAT-CONANP. 2007. Programa de conservación y manejo. Parque Nacional Nevado de Colima. Comisión Nacional de Áreas Naturales Protegidas y Gobierno de Jalisco. Guadalajara, México. 196 pp.

Shepard, G., D. Arora y A. Lampman. 2008. The grace of the flood: classification and use of wild mushrooms among the highland Maya of Chiapas. Economic Botany 62: 437-470. DOI: https://doi.org/10.1007/s12231-008-9044-5

Smith, A. H. 1972. The North American Species of Psathyrella. Memoirs of The New York Botanical Garden 24: 1-521.

Smith, A. H. 1975. A field guide to western Mushrooms. University of Michigan Press. Michigan, USA. 280 pp. 
Smith, A. y H. Thiers. 1964. A contribution toward a monograph of North American species of Suillus. Ann Arbor. Michigan, USA. 116 pp.

Téllez, C., L. Guzmán-Dávalos y G. Guzmán. 1988. Contribución al conocimiento de los hongos de la Reserva de la Biosfera de la Sierra de Manantlán, Jalisco. Revista Mexicana de Micología 4: 123-130.

Terríquez Villanueva, A. K., M. J. Herrera-Fonseca y O. Rodríguez Alcántar. 2017. Contribución al conocimiento de la micobiota del cerro Punta Grande, Mezcala, municipio de Poncitlán, Jalisco, México. Scientia Fungorum 45: 53-66.

UICN. 2020. Categorías y Criterios de la Lista Roja de la UICN: Versión 2020-1. Cambridge, UK. https://www.iucnredlist. org/search?query=fungi\&searchType=species (consultado abril de 2020).

Uitzil-Colli, M. O. y L. Guzmán-Dávalos. 2019. El género Morchella (Pezizales, Ascomycota) en Jalisco, México. Scientia Fungorum 49: 1-8. DOI: https://doi.org/10.33885/ sf.2019.49.1209

Valenzuela, R., G. Guzmán y J. Castillo. 1981. Descripciones de especies de macromicetes poco conocidas en México, con discusiones sobre su ecología y distribución. Boletín de la Sociedad Mexicana de Micología 15: 67-120.

Valenzuela, R., R. Nava y J. Cifuentes. 1994. El género Albatrellus en México 1. Revista Mexicana de Micología 10: 113-152.

Valenzuela, R., T. Raymundo y M. R. Palacios. 2004. Macromicetos que crecen sobre Abies religiosa en el Eje Neovolcánico Transversal. Polibotánica 18: 33-51.

Vázquez-González, L. S. y L. Guzmán-Dávalos. 1988. Algunas especies de hongos de la Barranca de Huentitán, estado de Jalisco. Revista Mexicana de Micología 4: 75-88.
Vázquez-González, L. S. y L. Guzmán-Dávalos. 1991. Los hongos del género Volvariella (Agaricales, Basidiomycetes) conocidos en Jalisco. Boletín IBUG 1(1): 15-22.

Vellinga, E. C. 1998. Glossary. In: Bas, C., T. H. Kuyper, M. E. Noordeloos y E. C. Vellinga (eds.). Flora agaricina Neerlandica, Vol. 1. Critical monographs on families of agarics and boleti occurring in the Netherlands. Balkema. Rhotterdam, The Netherlands. 182 pp.

Vellinga, E. C. 2001. Studies in Lepiota IV. Lepiota cristata and L. castaneidisca. Mycotaxon 80: 297-306.

Villarruel-Ordaz, J. L., E. Canseco-Zorrilla y J. Cifuentes. 2015. Diversidad fúngica en el municipio de San Gabriel Mixtepec, región Costa de Oaxaca. Revista Mexicana de Micología 41: 55-63.

Wijayawardene, N. N., K. D. Hyde, H. T. Lumbsch, J. Kuin-Liu, S. S. N. Maharachchikumbura, A. H. Ekanayaka, Q. Tian y R. Phookamsak. 2018. Outline of Ascomycota: 2017. Fungal Diversity 88: 167-263. DOI: https://doi.org/10.1007/ s13225-018-0394-8

Yei-Zeng, W. 2001. Some pyrophilous Discomycetes (Pezizales) in Taiwan. Bulletin of National Museum of Natural Science 14: 105-111.

Zarco, J. 1986. Estudio de la distribución ecológica de los hongos (principalmente macromicetos) en el Valle de México, basado en los especímenes depositados en el herbario ENCB. Revista Mexicana de Micología 2: 41-72. 


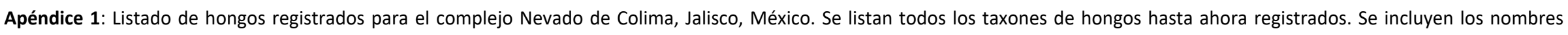

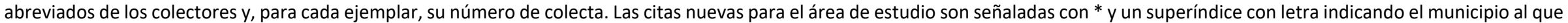

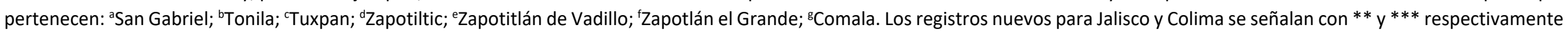

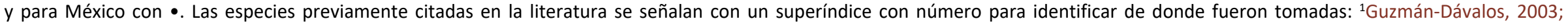

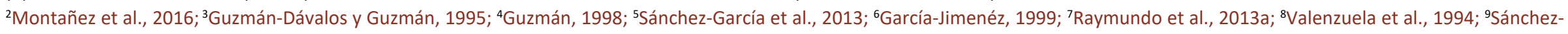

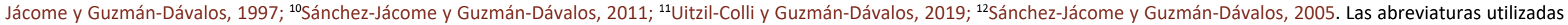

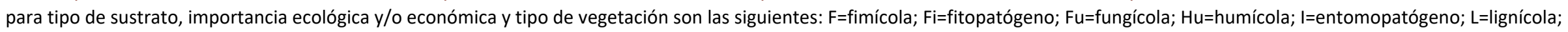

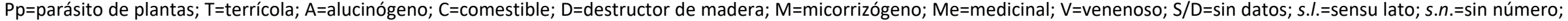

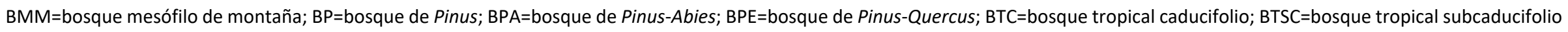

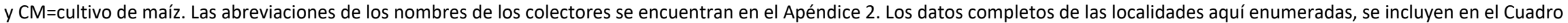
1. Todos los ejemplares se encuentran depositados en la Colección Micológica del Herbario IBUG.

\begin{tabular}{|c|c|c|c|c|c|}
\hline & Sustrato & Importancia & Colector & Tipo de vegetación & Localidad \\
\hline \multicolumn{6}{|l|}{ ASCOMYCOTA } \\
\hline \multicolumn{6}{|l|}{ LEOTIOMYCETES } \\
\hline \multicolumn{6}{|l|}{ HELOTIALES } \\
\hline \multicolumn{6}{|l|}{ CHLOROCIBORIACEAE } \\
\hline $\begin{array}{l}{ }^{* f, * * * \mathrm{~g} C h l o r o c i b o r i a ~ a e r u g i n a s c e n s ~(N y l .) ~ K a n o u s e ~ e x ~ C . S . ~} \\
\text { Ramamurthi, Korf \& L.R. Batra }\end{array}$ & $\mathrm{L}$ & & CT 960, CTP 199 & BMM, BPE & 1,38 \\
\hline \multicolumn{6}{|l|}{ LACHNACEAE } \\
\hline Erioscyphella abnormis (Mont.) Baral, Šandová \& B. Perić & $\mathrm{L}$ & & $A L 17$ & BPE & 1 \\
\hline \multicolumn{6}{|l|}{ LEOTIALES } \\
\hline \multicolumn{6}{|l|}{ BULGARIACEAE } \\
\hline${ }^{* * \mathrm{f}}$ Bulgaria inquinans (Pers.) Fr. & $\mathrm{L}$ & & LAF 5 & BPE & 1 \\
\hline \multicolumn{6}{|l|}{ LEOTIACEAE } \\
\hline *f Leotia lubrica (Scop.) Pers. & $\mathrm{T}$ & $\mathrm{M}, \mathrm{Me}$ & JPDN 6, SGCO 13 & BPE & 1 \\
\hline \multicolumn{6}{|l|}{ PEZIZOMYCETES } \\
\hline \multicolumn{6}{|l|}{ PEZIZALES } \\
\hline \multicolumn{6}{|l|}{ DISCINACEAE } \\
\hline Discina melaleuca Bres. & $\mathrm{T}$ & & ATs.n. & $\mathrm{BP}$ & 7 \\
\hline *fGyromitra infula (Schaeff.) Quél. & $\mathrm{T}$ & $\mathrm{M}, \mathrm{V}$ & CTP 403, YA 85 & $\mathrm{BP}, \mathrm{BPE}$ & 1,4 \\
\hline \multicolumn{6}{|l|}{ HELVELLACEAE } \\
\hline${ }^{10}$ Helvella acetabulum (L.) Quél. & $\mathrm{T}$ & $C, M$ & CDL s.n., MRSJ 948 & $\mathrm{BPE}, \mathrm{SD}$ & 1,20 \\
\hline *a,e Helvella crispa (Scop.) Fr. & $\mathrm{T}$ & $\mathrm{C}, \mathrm{M}, \mathrm{Me}$ & $\begin{array}{c}\text { AT s.n., CTP 359, FJT 584, MH 766, } \\
\text { OR } 1991\end{array}$ & $\mathrm{BP}, \mathrm{BPA}, \mathrm{BPE}$ & $\begin{array}{c}1,6,7,9,19 \\
28\end{array}$ \\
\hline Helvella elastica Bull. & $\mathrm{T}$ & $\mathrm{C}, \mathrm{M}$ & MH 489 & BPE & 1 \\
\hline *a,10Helvella lacunosa Afzel. & $\mathrm{T}$ & $\mathrm{C}, \mathrm{M}, \mathrm{Me}$ & FL 3401, MH 722, OR 1992 & $B P A, B P E, S D$ & $\begin{array}{c}1,6,9,20 \\
27,28\end{array}$ \\
\hline *e,10Helvella macropus (Pers.) P. Karst. & $\mathrm{T}$ & $C, M$ & MH 697, RMA s.n., RZV 317 & BMM, BPA, BPE, SD & $1,6,14,27$ \\
\hline
\end{tabular}


Apéndice 1. Continuación.

\begin{tabular}{|c|c|c|c|c|c|}
\hline & Sustrato & Importancia & Colector & Tipo de vegetación & Localidad \\
\hline Helvella subglabra N.S. Weber & $\mathrm{T}$ & $C, M$ & FJT 589 & BPE & 1 \\
\hline \multicolumn{6}{|l|}{ MORCHELLACEAE } \\
\hline${ }^{11}$ Morchella complejo angusticeps Peck & $\mathrm{T}$ & $\mathrm{C}, \mathrm{M}$ & TPF 8 & BPE & 1 \\
\hline${ }^{11}$ Morchella complejo costata Pers. & $\mathrm{T}$ & $C, M$ & FL 3402 & BPE & 1 \\
\hline Morchella complejo esculenta (L.) Pers. & $\mathrm{T}$ & $\mathrm{C}, \mathrm{M}, \mathrm{Me}$ & $B C D 24$ & BPE & 1 \\
\hline${ }^{11}$ Morchella guatemalensis Guzmán, M.F. Torres \& Logem. & $\mathrm{T}$ & $\mathrm{C}, \mathrm{M}$ & LGD 7547 & BPE & 1 \\
\hline \multicolumn{6}{|l|}{ PEZIZACEAE } \\
\hline${ }^{1}$ Peziza atrovinosa Cooke \& W.R. Gerard & $\mathrm{T}$ & & LGD 4212 & BPE & 1 \\
\hline Peziza badia Pers. & $\mathrm{Hu}$ & C & MRSJ 947 & BPE & 1,9 \\
\hline${ }^{1}$ Peziza howsei Roze \& Boud. & $\mathrm{Hu}$ & & LGD 2164 & BPE & 1 \\
\hline${ }^{12}$ Peziza praetervisa Bres. & $\mathrm{T}$ & & LGD 3570 & BPE & 9 \\
\hline \multicolumn{6}{|l|}{ PYRONEMATACEAE } \\
\hline${ }^{* \mathrm{f}}$ Aleuria aurantia (Pers.) Fuckel & $\mathrm{L}$ & C & OR 698 & BPE & 1 \\
\hline${ }^{1}$ Geopyxis carbonaria (Alb. \& Schwein.) Sacc. & $\mathrm{T}$ & & IA 1115 & BPE & 1 \\
\hline${ }^{* f}$ Humaria hemisphaerica (F.H. Wigg.) Fuckel & $\mathrm{T}$ & & AT s.n., CTP 445 & BP, BPE & 1,7 \\
\hline${ }^{10}$ Otidea alutacea (Pers.) Massee & $\mathrm{Hu}$ & & ECE 18 & BPE, SD & 1,20 \\
\hline Otidea alutacea var. microspora Kanouse & $\mathrm{Hu}$ & & DGS 448, MRSJ 943 & BMM, BPE & 1,15 \\
\hline${ }^{10}$ Otidea onotica (Pers.) Fuckel & $\mathrm{Hu}$ & C & AT s.n. & BPE, SD & $7,20,27$ \\
\hline${ }^{*}$ Scutellinia scutellata (L.) Lambotte & $\mathrm{Hu}$ & & CTP 394, MH 1617 & BMM, BPE & 1,14 \\
\hline Sowerbyella rhenana (Fuckel) J. Moravec & $\mathrm{Hu}, \mathrm{L}$ & & APA 17 & BPE & 1 \\
\hline Tarzetta catinus (Holmsk.) Korf \& J.K. Rogers & $\mathrm{T}$ & & $A L 188-D$ & BPE & 1 \\
\hline${ }^{1}$ Trichophaea woolhopeia (Cooke \& W. Phillips) Boud. & $\mathrm{T}$ & & AT s.n. & BPE & 1 \\
\hline \multicolumn{6}{|l|}{ RHIZINACEAE } \\
\hline${ }^{* * \mathrm{e}}$ Rhizina undulata Fr. & $\mathrm{Hu}$ & & $A H 27$ & BPE & 16 \\
\hline \multicolumn{6}{|l|}{ SARCOSCYPHACEAE } \\
\hline Microstoma floccosum (Sacc.) Raitv. & $\mathrm{L}$ & & HEFL s.n., KE 39 & BPE & 1,2 \\
\hline$* \mathrm{f}, * * * \mathrm{~g}$ Sarcoscypha coccinea (Gray) Boud. & $\mathrm{L}$ & & DGBA 3, CTP 163 & BMM, BPE & 1,37 \\
\hline \multicolumn{6}{|l|}{ SORDARIOMYCETES } \\
\hline \multicolumn{6}{|l|}{ HYPOCREALES } \\
\hline \multicolumn{6}{|l|}{ CORDYCIPITACEAE } \\
\hline${ }^{* \mathrm{f} C o r d y c e p s ~ m i l i t a r i s ~(L .) ~ F r . ~}$ & 1 & Me & CA 25 & BPE & 1 \\
\hline
\end{tabular}


Sustrato

Importancia

Colector

Tipo de vegetación

Localidad

HYPOCREACEAE

${ }^{* a, 10}$ Hypomyces chrysospermus Tul. \& C. Tul.

$\mathrm{Fu}$

${ }^{* f}$ Hypomyces hyalinus (Schwein.) Tul. \& C. Tul.

${ }^{10}$ Hypomyces lactifluorum (Schwein.) Tul. \& C. Tul.

**e,fTrichoderma alutaceum Jaklitsch

Trichoderma viride Pers.

\section{OPHIOCORDYCIPITACEAE}

Tolypocladium capitatum (Holmsk.) C.A. Quandt, Kepler \& Spatafora

XYLARIALES

\section{HYPOXYLACEAE}

Annulohypoxylon thouarsianum (Lév.) Y.M. Ju, J.D. Rogers \&

H.M. Hsieh

${ }^{*}$ Daldinia cf. childiae J.D. Rogers \&Y.M. Ju

${ }^{10}$ Daldinia macrospora F. San Martín, Y.M. Ju \& J.D. Rogers

***8Daldinia vernicosa Ces. \& De Not.

\section{XYLARIACEAE}

${ }^{*}$ Xylaria grammica (Mont.) Mont.

*gXylaria hypoxylon (L.) Grev.

${ }^{*}$ Xylaria multiplex (Kunze) Fr.

${ }^{*}$ Xylaria polymorpha (Pers.) Grev.

\section{BASIDIOMYCOTA}

\section{AGARICOMYCETES}

\section{AGARICALES}

\section{AGARICACEAE}

*a Agaricus arvensis Schaeff.

${ }^{* f}$ Agaricus campestris L.

*a,10 Agaricus placomyces Peck

Agaricus sylvaticus Schaeff.

*e,fAgaricus xanthodermus Genev.

Bovista brunnea Berk.

Bovista fusca Lév.

${ }^{10}$ Bovista leucoderma Kreisel
AT s.n., CTP 99, MH 725, OR 1990

BPA, BP, BPE, SD

, 6, 7, 9, 20,

27,28

AF 1, GG 24615

BPE

BPA, BPE, SD

$B P, B P E$

$B P$

MRSJ 108

GG 32590

BPE

1,23

$1,3,6,20,27$

4,19

34

1

MH 492, OR 735

BPE

1,3
$1,5,14,28$

12

1,37

1, 6

$1,2,34,38$

1

1
CTP 212, OR 1589, 4000

SD

OR 3100, 4168, RZV 505

MH 759, OV 43

CTP 197, 478-A, OV 148

GA 34

AC 5, MAG 8

BMM, BPE

SD

BMM, BPE

BPA, BPE

$B M M, B P, B P E$

BPE

BPE

\section{GG 22003 \\ CT 964 \\ OR 801}

AL 165, MRSJ 176

VMLQ 7, OR 4015

JNR 8082, 8086, 8088

GP 5, OV 548, VC 3

$\begin{array}{cc}\text { BPE } & 1,32 \\ \text { BPE } & 1 \\ \text { BPE, SD } & 12,31 \\ \text { BPE } & 1 \\ \text { BMM, BPE } & 1,14 \\ \text { BPE } & 1 \\ \text { BPE } & 1 \\ \text { SD } & 20\end{array}$




\begin{tabular}{|c|c|c|c|c|c|}
\hline & Sustrato & Importancia & Colector & Tipo de vegetación & Localidad \\
\hline Bovista pusilla (Batsch) Pers. & $\mathrm{T}$ & Me & MF 14 & BPE & 1 \\
\hline${ }^{*, \mathrm{fg}}$ Chlorophyllum molybdites (G. Mey.) Massee & $\mathrm{T}$ & $\mathrm{V}$ & CTP 190, GG 24644 & BMM, BPE & 1,38 \\
\hline Chlorophyllum rhacodes (Vittad.) Vellinga & $\mathrm{T}$ & $\mathrm{C}$ & GGa s.n. & BPE & 1 \\
\hline *a,f,10 Coprinus comatus (O.F. Müll.) Pers. & $\mathrm{T}$ & $\mathrm{C}$ & OHV 25, OR 4114 & $B P, B P E, S D$ & $1,27,34$ \\
\hline${ }^{* \mathrm{f} C y a t h u s ~ o l l a ~(B a t s c h) ~ P e r s . ~}$ & $\mathrm{~F}$ & & FLP 40, MA 33 & BPE & 1 \\
\hline${ }^{* \mathrm{f} C}$ ystoderma amianthinum (Scop.) Fayod & $\mathrm{T}$ & & SYR 117 & BPE & 1 \\
\hline${ }^{* \mathrm{f}}$ Echinoderma asperum (Pers.) Bon & $\mathrm{Hu}$ & $\mathrm{V}$ & CTP 112, EL 11 & BPE & 1 \\
\hline${ }^{10}$ Floccularia luteovirens (Alb. \& Schwein.) Pouzar & $\mathrm{T}$ & $\mathrm{C}$ & SD & SD & 12 \\
\hline *a Lepiota alopochroa (Berk. \& Broome) Sacc. & $\mathrm{T}$ & & OR 1977, OV 540 & BPE & 1,28 \\
\hline *a Lepiota clypeolaria (Bull.) P. Kumm. & $\mathrm{T}$ & & MLF 838, OR 1591 & BPE & $1,3,28$ \\
\hline${ }^{* * \mathrm{f}}$ Lepiota cristata (Bolton) P. Kumm. & $\mathrm{Hu}, \mathrm{T}$ & $\mathrm{V}$ & CTP 207 & BPE & 5 \\
\hline$* * * \mathrm{~g}$ Leucoagaricus meleagris (Gray) Singer & $\mathrm{T}$ & & CTP 188 & BMM & 38 \\
\hline *a Leucoagaricus rubrotinctus (Peck) Singer & $\mathrm{T}$ & $\mathrm{V}$ & OR 800 & BPE & 31 \\
\hline${ }^{10}$ Leucocoprinus cepistipes (Sowerby) Pat. & $\mathrm{L}$ & & SD & SD & 27 \\
\hline *a Lycoperdon candidum Pers. & $\mathrm{T}$ & $\mathrm{C}, \mathrm{Me}$ & CTP 71, IA 819 & BPE & 1,29 \\
\hline *a,fLycoperdon molle Pers. & $\mathrm{T}$ & & AL 175, IA 822 & BPE & 1,29 \\
\hline Lycoperdon perlatum Pers. & $\mathrm{T}$ & $\mathrm{C}, \mathrm{M}, \mathrm{Me}$ & AT s.n., CTP 337, HRRR 28 & BPA, BPE & $1,6,9,19$ \\
\hline${ }^{* a, 10}$ Lycoperdon pyriforme Schaeff. & L & $\mathrm{C}, \mathrm{Me}$ & CTP 389, DGS 404, RL s.n. & BMM, BPE, SD & $1,15,27,32$ \\
\hline Lycoperdon umbrinum Pers. & $\mathrm{T}$ & $\mathrm{C}, \mathrm{Me}$ & AT s.n., CTP 486 & $\mathrm{BP}, \mathrm{BPE}$ & 1,7 \\
\hline${ }^{* \mathrm{f}}$ Macrolepiota procera (Scop.) Singer & $\mathrm{T}$ & $\mathrm{C}, \mathrm{Me}$ & MLF 836 & BPE & 1 \\
\hline Micropsalliota heinemaniana Guzm.-Dáv. & $\mathrm{Hu}$ & & OV 439 & BPE & 1 \\
\hline Micropsalliota subalpina Guzm.-Dáv. \& Heinem. & $\mathrm{Hu}$ & & JS 79 & BPE & 1 \\
\hline${ }^{* \mathrm{~d}}$ Montagnea arenaria (DC.) Zeller & $\mathrm{T}$ & & OR 4403 & BTC & 21 \\
\hline${ }^{* f}$ Phaeolepiota aurea (Matt.) Maire & L & $A, V$ & CFMM 7 & BPE & 1 \\
\hline \multicolumn{6}{|l|}{ AMANITACEAE } \\
\hline${ }^{* \mathrm{f} A m a n i t a}$ alexandri Guzmán & $\mathrm{T}$ & $\mathrm{M}, \mathrm{V}$ & SGCO 9 & BPE & 1 \\
\hline Amanita cokeri E.-J. Gilbert \& Kühner ex E.-J. Gilbert & $\mathrm{T}$ & $\mathrm{M}, \mathrm{V}$ & GG 11942 & BPE & 11 \\
\hline${ }^{10}$ Amanita complejo caesarea (Scop.) Pers. & $\mathrm{T}$ & $\mathrm{C}, \mathrm{M}, \mathrm{Me}$ & OR 733 & BPE, SD & $1,3,27$ \\
\hline${ }^{* \mathrm{f}, 10}$ Amanita flavoconia G.F. Atk. & $\mathrm{T}$ & M & YAGA 14 & BPE, SD & 1,27 \\
\hline${ }^{* a}$ Amanita fulva Fr. & $\mathrm{T}$ & $C, M$ & MRSJ $113, R G C 12$ & $\mathrm{BP}, \mathrm{BPE}$ & 1,34 \\
\hline${ }^{* * * \mathrm{~g}}$ Amanita gemmata (Fr.) Bertill. & $\mathrm{T}$ & $\mathrm{M}, \mathrm{V}$ & CTP 124, 182, 362 & BMM, BPE & $1,2,37$ \\
\hline${ }^{* \mathrm{f} A m a n i t a ~ g e m m a t a}$ f. gemmata (Fr.) Bertill. & $\mathrm{T}$ & $\mathrm{M}, \mathrm{V}$ & MG 33 & BPE & 1 \\
\hline${ }^{* \mathrm{f}}$ Amanita magnivelaris Peck & $\mathrm{T}$ & $\mathrm{M}, \mathrm{V}$ & LSV 402, MH 495 & BPE & 1 \\
\hline
\end{tabular}




\begin{tabular}{|c|c|c|c|c|c|}
\hline & Sustrato & Importancia & Colector & Tipo de vegetación & Localidad \\
\hline${ }^{10}$ Amanita muscaria (L.) Lam. & $\mathrm{T}$ & $\mathrm{M}, \mathrm{Me}, \mathrm{V}$ & CTP 502, JA s.n., MCSG 5 & BPE, SD & $1,2,27$ \\
\hline${ }^{* \mathrm{f}}$ Amanita muscaria var. flavivolvata (Singer) D.T. Jenkins & $\mathrm{T}$ & $\mathrm{M}, \mathrm{Me}$ & GN 263, MLF 835 & BPE & 1 \\
\hline${ }^{10}$ Amanita pantherina (DC.) Krombh. & $\mathrm{T}$ & $\mathrm{M}, \mathrm{V}$ & EF 561, MRSJ 615 & BPA, BPE, SD & $1,6,27$ \\
\hline${ }^{* \mathrm{f}}$ Amanita cf. ponderosa Malençon \& R. Heim & $\mathrm{T}$ & $\mathrm{C}, \mathrm{M}$ & LSV 650 & BPE & 1 \\
\hline${ }^{* e, f, 10}$ Amanita rubescens Pers. & $\mathrm{T}$ & $\mathrm{C}, \mathrm{M}, \mathrm{Me}$ & MAHR 31, OR 4071 & BMM, BPE, SD & $1,14,27$ \\
\hline${ }^{* a}$ Amanita solitaria (Bull.) Fr. & $\mathrm{T}$ & M & OR 1595 & BPE & 28 \\
\hline${ }^{* \mathrm{f}}$ Amanita tuza Guzmán & $\mathrm{T}$ & $\mathrm{C}, \mathrm{M}$ & IA 1109 & BPE & 1 \\
\hline${ }^{* a, 10}$ Amanita vaginata (Bull.) Lam. & $\mathrm{T}$ & $\mathrm{C}, \mathrm{M}, \mathrm{Me}$ & CTP 439, DFG 9, MRSJ 249 & $\mathrm{BPE}, \mathrm{SD}$ & $1,27,30$ \\
\hline Amanita valens (E.-J. Gilbert) Bertault & $\mathrm{T}$ & $\mathrm{C}, \mathrm{M}$ & GG 11959 & BPE & 11 \\
\hline Amanita verna (Bull.) Lam. & $\mathrm{T}$ & $\mathrm{M}, \mathrm{Me}, \mathrm{V}$ & MG 37 & BPE & 1 \\
\hline${ }^{* \mathrm{f} A m a n i t a}$ virosa Bertill. & $\mathrm{T}$ & $\mathrm{M}, \mathrm{Me}, \mathrm{V}$ & MH 1486, RGC 6 & BPE & 1 \\
\hline \multicolumn{6}{|l|}{ BOLBITIACEAE } \\
\hline${ }^{* \mathrm{f}}$ Bolbitius titubans (Bull.) Fr. & $\mathrm{T}$ & & GT 32 & BPE & 1 \\
\hline \multicolumn{6}{|l|}{ CORTINARIACEAE } \\
\hline${ }^{* \mathrm{f} C o r t i n a r i u s ~ s a n g u i n e u s ~(W u l f e n) ~ G r a y ~}$ & $\mathrm{~T}$ & $M, V$ & NGRO 9 & BPE & 1 \\
\hline${ }^{* \mathrm{f} C o r t i n a r i u s ~ s e m i s a n g u i n e u s ~(F r .) ~ G i l l e t ~}$ & $\mathrm{~T}$ & $\mathrm{M}, \mathrm{V}$ & $A A 12$ & BPE & 1 \\
\hline${ }^{* a, 10}$ Cortinarius splendens Rob. Henry & $\mathrm{T}$ & $\mathrm{M}, \mathrm{V}$ & OR 745 & BPE, SD & 12,29 \\
\hline \multicolumn{6}{|l|}{ CYPHELLACEAE } \\
\hline${ }^{*}$ Chondrostereum purpureum (Pers.) Pouzar & L & & MH 1653, RZV 311 & BMM & 14 \\
\hline Gloeostereum incarnatum S. Ito \& S. Imai & L & C & MH 695 & BPE & 1 \\
\hline \multicolumn{6}{|l|}{ ENTOLOMATACEAE } \\
\hline Entoloma cf. carolinianum Hesler & $\mathrm{T}$ & & CM 16, DM 222, OR 1590 & BPE & 1 \\
\hline${ }^{2}$ Entoloma chytrophilum Wölfel, Noordel. \& Dähncke & L & & OV 443 & BPE & 1 \\
\hline${ }^{* \mathrm{f} E n t o l o m a ~ c l y p e a t u m ~(L .) ~ P . ~ K u m m . ~}$ & $\mathrm{~T}$ & $\mathrm{C}, \mathrm{M}$ & CTP 527, LERG 32 & BPE & 1 \\
\hline $\begin{array}{l}\text { Entoloma griseorhodopolium Montañez, Noordel. \& Guzm.- } \\
\text { Dáv. }\end{array}$ & $\mathrm{T}$ & & OV 528 & BPE & 1 \\
\hline $\begin{array}{l}\text { Entoloma minutisporum (Vila \& Llimona) J. Carbó, Vila, } \\
\text { Català \& F. Caball. }\end{array}$ & $\mathrm{Hu}$ & & LGD 5432 & BPE & 1 \\
\hline Entoloma sericeum Quél. & $\mathrm{T}$ & V & DM 214 & BPE & 1 \\
\hline${ }^{2}$ Entoloma spodopus Montañez, Noordel. \& Guzm.-Dáv. & $\mathrm{T}$ & & YA 96 & BPE & 1 \\
\hline${ }^{* d, 2}$ Entoloma cf. subcoelestinum (Largent) Blanco-Dios & $\mathrm{T}$ & & AGJ 28, CTP 259 & BPE, BTC & 1,21 \\
\hline \multicolumn{6}{|l|}{ FISTULINACEAE } \\
\hline$* \mathrm{f}, * * * \mathrm{~g}$ Porodisculus pendulus (Fr.) Murrill & L & & CTP 13, OR 3107 & BMM, BPE & 1,37 \\
\hline${ }^{* f}$ Pseudofistulina radicata (Schwein.) Burds. & $\mathrm{L}, \mathrm{Pp}$ & & CTP 268 & BPE & 1 \\
\hline
\end{tabular}




\begin{tabular}{|c|c|c|c|c|c|}
\hline & Sustrato & Importancia & Colector & Tipo de vegetación & Localidad \\
\hline \multicolumn{6}{|l|}{ HYDNANGIACEAE } \\
\hline Laccaria amethystina Cooke & $\mathrm{T}$ & $C, M$ & $\begin{array}{c}\text { AT s.n., CTP 350, DFG 7, OR 4378, } \\
3964\end{array}$ & $\mathrm{BMM}, \mathrm{BP}, \mathrm{BPE}$ & $\begin{array}{c}1,2,7,8,9 \\
14,19\end{array}$ \\
\hline *e,10 Laccaria laccata (Scop.) Cooke & $\mathrm{T}$ & $C, M$ & $\begin{array}{c}\text { AT s.n., FB 153, JMRA 32, OR 4166, } \\
3965\end{array}$ & BMM, BP, BPE, SD & $1,2,7,14,27$ \\
\hline *faccaria ochropurpurea (Berk.) Peck & $\mathrm{T}$ & $\mathrm{C}, \mathrm{M}$ & CTP 541, 567 & BPE & 1 \\
\hline \multicolumn{6}{|l|}{ HYGROPHORACEAE } \\
\hline $\begin{array}{l}{ }^{* f} \text { Ampulloclitocybe clavipes (Pers.) Redhead, Lutzoni, } \\
\text { Moncalvo \& Vilgalys }\end{array}$ & $\mathrm{T}$ & $\mathrm{C}, \mathrm{M}$ & OV 535 & BPE & 1 \\
\hline *e,fHygrocybe conica (Schaeff.) P. Kumm. & $\mathrm{T}$ & & CTP 74, 366, RZV 290 & BMM, BPE & $1,2,14$ \\
\hline **aHygrocybe spadicea (Scop.) P. Karst. & $\mathrm{Hu}$ & & MRSJ 107 & $\mathrm{BP}$ & 34 \\
\hline *a Hygrophorus russula (Schaeff. ex Fr.) Kauffman & $\mathrm{T}$ & $\mathrm{C}, \mathrm{M}$ & DAJR s.n., OR 742 & BPE & 1,29 \\
\hline \multicolumn{6}{|l|}{ HYMENOGASTRACEAE } \\
\hline ***gGalerina subochracea A.H. Sm. & L & $\mathrm{V}$ & CTP 159 & BMM & 37 \\
\hline${ }^{3}$ Gymnopilus capitatus Guzm.-Dáv. \& Guzmán & L & & LGD 5296 & BP & 34 \\
\hline${ }^{3}$ Gymnopilus nevadensis Guzm.-Dáv. \& Guzmán & $\mathrm{L}$ & & LGD 3469 & BPE & 1 \\
\hline${ }^{* \mathrm{f} G y m n o p i l u s ~ p e n e t r a n s ~(F r .) ~ M u r r i l ~}$ & $\mathrm{~L}$ & $\mathrm{~V}$ & CTP 143, 525, DFG 5, MRSJ 570 & BPE & 1 \\
\hline **f Gymnopilus sapineus (Fr.) Murrill & L & & CTP 570 & BPE & 1 \\
\hline${ }^{3}$ Gymnopilus subpurpuratus Guzm.-Dáv. \& Guzmán & L & & LGD 4250 & BPE & 1 \\
\hline ***gGymnopilus tuxtlensis Guzm.-Dáv. & L & & CTP 156, 157 & BMM & 37 \\
\hline${ }^{10}$ Hypholoma fasciculare (Huds.) P. Kumm. & $\mathrm{L}$ & Me, V & GN 270, MH 762, RRD 2835 & BPA, BPE, SD & $1,6,9,27$ \\
\hline *apsilocybe cubensis (Earle) Singer & $\mathrm{F}$ & $\mathrm{A}, \mathrm{Me}$ & JPL s.n., OR 1986 & BPE & 1,28 \\
\hline${ }^{10}$ Psilocybe mexicana R. Heim & $\mathrm{F}$ & $\mathrm{A}, \mathrm{Me}$ & SD & SD & 27 \\
\hline${ }^{10}$ Psilocybe subcubensis Guzmán & $\mathrm{F}$ & $\mathrm{A}, \mathrm{Me}$ & SD & SD & 27 \\
\hline 4,10Psilocybe zapotecorum R. Heim & $\mathrm{F}$ & $\mathrm{A}, \mathrm{Me}$ & LGD 2144 & BPE, SD & 1,27 \\
\hline \multicolumn{6}{|l|}{ INOCYBACEAE } \\
\hline${ }^{* * \mathrm{f} C r e p i d o t u s ~ a p p l a n a t u s ~(P e r s .) ~ P . ~ K u m m . ~}$ & L & & CTP 218 & BPE & 5 \\
\hline Crepidotus calolepis (Fr.) P. Karst. & L & & CTP 17, 92, 115 & BPE & 1 \\
\hline${ }^{* a}$ Crepidotus cinnabarinus Peck & $\mathrm{L}$ & & MRSJ 181, OR 741 & BPE & 1,29 \\
\hline *a,f Crepidotus mollis (Schaeff.) Staude & L & & MRSJ 272, OR 695 & BPE & 1,30 \\
\hline Crepidotus uber (Berk. \& M.A. Curtis) Sacc. & $\mathrm{L}$ & & GL 5 & BPE & 1 \\
\hline *f/nocybe ayeri Furrer-Ziogas. & $\mathrm{T}$ & & CTP 375 & $\mathrm{BP}$ & 4 \\
\hline *f/nocybe geophylla (Bull.) P. Kumm. & $\mathrm{T}$ & $M, V$ & AT s.n., MH 476 & $B P, B P E$ & 1,7 \\
\hline
\end{tabular}




\begin{tabular}{|c|c|c|c|c|c|}
\hline & Sustrato & Importancia & Colector & Tipo de vegetación & Localidad \\
\hline *f/nocybe geophylla var. alba Gillet & $\mathrm{T}$ & $M, V$ & SYR 127 & BPE & 1 \\
\hline *f,10/nocybe geophylla var. lilacina (Peck) Gillet & $\mathrm{T}$ & $M, V$ & CTP 553, DFG 11, DM 224 & BPE, SD & 1,27 \\
\hline **f/nocybe obsoleta (Quadr. \& Lunghini) Valade & $\mathrm{T}$ & & CTP 262 & BPE & 1 \\
\hline *a,f Inocybe rimosa (Bull.) P. Kumm. & $\mathrm{T}$ & M & GN 307, oV 430 & BPE & 1,30 \\
\hline \multicolumn{6}{|l|}{ LYOPHYLLACEAE } \\
\hline Lyophyllum decastes (Fr.) Singer & $\mathrm{T}$ & $\mathrm{C}, \mathrm{Me}$ & OR 4118-B & BPE & 1 \\
\hline \multicolumn{6}{|l|}{ MARASMIACEAE } \\
\hline${ }^{10}$ Baeospora myosura (Fr.) Singer & L & & SYR 198 & BPA, SD & 6,27 \\
\hline${ }^{* f, 10}$ Marasmius guzmanianus Singer & $\mathrm{Hu}$ & & BAAO 571, CTP 102, RRD 2840 & $B P, B P E, S D$ & $1,4,9,27$ \\
\hline${ }^{* f}$ Marasmius plicatulus Peck & $\mathrm{Hu}$ & & CT 943 & BPE & 1 \\
\hline${ }^{10}$ Marasmius rotula (Scop.) Fr. & $\mathrm{Hu}$ & & CTP 125, 225 & BPE, SD & $1,5,27$ \\
\hline \multicolumn{6}{|l|}{ MYCENACEAE } \\
\hline${ }^{* \mathrm{f}}$ Mycena epipterygia (Scop.) Gray & $\mathrm{Hu}$ & & SF 9 & BPE & 1 \\
\hline *aMycena leaiana (Berk.) Sacc. & L & & CTP 20, OR 1982 & BPE & 1,28 \\
\hline *f,10Mycena pura (Pers.) P. Kumm. & $\mathrm{Hu}$ & $\mathrm{C}$ & MRSJ 955, OR 3315 & BPE, SD & 1,20 \\
\hline *f,***g Panellus pusillus (Pers. ex Lév.) Burds. \& O.K. Mill. & L & Me & OR 3102, 4173 & BMM, BPE & 1,37 \\
\hline Panellus stipticus (Bull.) P. Karst. & L & & AL 209, DRDL 9 & BPE & 1 \\
\hline${ }^{* f}$ Xeromphalina campanella (Batsch) Kühner \& Maire & L & & CTP 77, MH 719 & BPA, BPE & 1,6 \\
\hline$* \mathrm{f}, * * * \mathrm{~g}$ Xeromphalina tenuipes (Schwein.) A.H. Sm. & L & & BAAO 432, CTP 63, OR 3101 & $\mathrm{BMM}, \mathrm{BP}, \mathrm{BPE}$ & $1,4,37$ \\
\hline \multicolumn{6}{|l|}{ OMPHALOTACEAE } \\
\hline $\begin{array}{l}{ }^{* f} \text { Connopus acervatus (Fr.) K.W. Hughes, Mather \& R.H. } \\
\text { Petersen }\end{array}$ & $\mathrm{Hu}, \mathrm{L}$ & C & JS 92 & $\mathrm{BP}, \mathrm{BPE}$ & 1,34 \\
\hline *fGymnopus alkalivirens (Singer) Halling & $\mathrm{Hu}$ & $\mathrm{V}$ & GA 41 & BPE & 1 \\
\hline *a,e Gymnopus dryophilus (Bull.) Murrill & $\mathrm{Hu}$ & C & GN 251, MRSJ 231, OR 4014 & BMM, BPE & $1,14,29$ \\
\hline${ }^{* \mathrm{f} G y m n o p u s ~ f u s i p e s ~(B u l l .) ~ G r a y ~}$ & L & & MLF 786 & BPE & 1 \\
\hline *a,eGymnopus polyphyllus (Peck) Halling & $\mathrm{Hu}$ & C & $\begin{array}{c}\text { CTP 341, MH 1627, MRSJ 248, LJOV } \\
22\end{array}$ & BMM, BPE & $1,14,19,31$ \\
\hline${ }^{* f}$ Lentinula boryana (Berk. \& Mont.) Pegler & $\mathrm{L}$ & C & $H R 6$, IA 524, OV 47 & BPE & 1 \\
\hline *fOmphalotus mexicanus Guzmán \& V. Mora & $\mathrm{T}$ & & CTP 368, DFG 13-A & BPE & 1,2 \\
\hline${ }^{10}$ Rhodocollybia butyracea (Bull.) Lennox & $\mathrm{Hu}$ & C & SD & SD & 27 \\
\hline Rhodocollybia maculata (Alb. \& Schwein.) Singer & $\mathrm{Hu}$ & & DFG 12, MRSJ 574 & BPE & 1,9 \\
\hline \multicolumn{6}{|l|}{ PHYSALACRIACEAE } \\
\hline *a,fArmillaria obscura (Schaeff.) Herink & $\mathrm{Pp}$ & C & CTP 18, GG 22001 & BPE & 1,32 \\
\hline${ }^{* f}$ Cyptotrama asprata (Berk.) Redhead \& Ginns & L & & CTP 14, OV 527, SG 4 & BPE & 1 \\
\hline
\end{tabular}




\section{*a,fOudemansiella canarii (Jungh.) Höhn.}

\section{PLEUROTACEAE}

Hohenbuehelia angustata (Berk.) Singer

${ }^{* \mathrm{f}}$ Hohenbuehelia grisea (Peck) Singer

*fHohenbuehelia nigra (Schwein.) Singer

${ }^{10}$ Hohenbuehelia petaloides (Bull.) Schulzer

**fHohenbuehelia spathulata (Pers.) Singer

*f,gPleurotus djamor (Rumph. ex Fr.) Boedijn

*fPleurotus dryinus (Pers.) P. Kumm.

\section{PLUTEACEAE}

Pluteus atromarginatus (Konrad) Kühner

Pluteus aurantiorugosus (Trog) Sacc.

*a,e Pluteus cervinus (Schaeff.) P. Kumm.

Pluteus chrysophlebius (Berk. \& M.A. Curtis) Sacc.

Pluteus harrisii Murril

Pluteus horakianus Rodr.-Alcánt.

Pluteus nevadensis Rodr.-Alcánt.

*fVolvopluteus gloiocephalus (DC.) Vizzini, Contu \& Justo

\section{PSATHYRELLACEAE}

Lacrymaria hypertropicalis (Guzmán, Bandala \& Montoya)

\section{Cortez}

*f Panaeolina foenisecii (Pers.) Maire

${ }^{*}$ Panaeolus antillarum (Fr.) Dennis

*fPanaeolus cyanescens Sacc.

${ }^{10}$ Panaeolus papilionaceus (Bull.) Quél.

Panaeolus semiovatus (Sowerby) S. Lundell \& Nannf.

*a,10 Psathyrella candolleana (Fr.) Maire

*e,f fsathyrella delineata (Peck) A.H. Sm.

-f Psathyrella longistriata (Murrill) A.H. Sm.

\section{SCHIZOPHYLLACEAE}

*c,d,e,gSchizophyllum commune Fr.

\section{Sustrato}

L

Importancia

C

CTP 84, MH 727, OR 1993

Tipo de vegetación

BPA, BPE

C

L

$\mathrm{T}$

L

$\mathrm{L}$

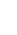

C, Me

CTP 24, 492
CTP 518
SD
CTP 511

LARR 5, SMM 18

IA 1919

GG 30554, OR 1971

C

C
A, V

Me
OV 525

GG 30554, OR 1971
OV 525
CTP 247, OR $1581,2020,4010$
OR 4119, OV 530
LSV 764,773
OR 4011
OV 525
CTP 147, OV 196

GG 29585-A

SG 31

RB 28

RISC 9

CVG 18

GGas.n.

CTP 69, 510, MRSJ 251, OR 1592

$$
\begin{aligned}
& \text { CT } 949 \\
& \text { GN } 276
\end{aligned}
$$

BPE

BMM

BPE

BPE

$\begin{array}{cc}\text { BPE } & 1 \\ \text { BPE } & 1,2 \\ \text { BPE } & 2 \\ \text { SD } & 27 \\ \text { BPE } & 2 \\ \text { MM, BPE } & 1,37 \\ \text { BPE } & 1\end{array}$

$\begin{array}{ll}\text { BPE } & 1 \\ \text { BPE } & 1\end{array}$

BMM, BPE $\quad 1,5,14,28$

BMM, BPE 1,37

1

14

1

$$
1
$$

$\begin{array}{ll}\text { BPE } & 1\end{array}$

BPE 1

$\begin{array}{ll}\text { BPE } & 1 \\ \text { BPE } & 1\end{array}$

BPE, SD $\quad 1,27$

BPE 1

$B P, B P E, S D \quad 1,2,20,28$,

BPE $\quad 30,34$

BPE $\quad 1$

AF 7, CTP 183, MH 718, OR 3115, BPA, BMM, BPE, BTC, 1, 2, 6, 14, 4387, OV 143, RIAB 31, RZV 312 BTSC 21, 23, 37, 


\begin{tabular}{|c|c|c|c|c|c|}
\hline & Sustrato & Importancia & Colector & Tipo de vegetación & Localidad \\
\hline *gSchizophyllum fasciatum Pat. & L & C & OR 2955 & BMM & 37 \\
\hline *fSchizophyllum umbrinum Berk. & L & & CTP 283 & BPE & 5 \\
\hline \multicolumn{6}{|l|}{ STROPHARIACEAE } \\
\hline${ }^{* f}$ Agrocybe pediades (Fr.) Fayod & $\mathrm{T}$ & & $C A 16, K M 7$ & BPE & 1 \\
\hline *a,fDeconica coprophila (Bull.) P. Karst. & $\mathrm{F}$ & & CT 962-A, IA 757 & $\mathrm{BP}, \mathrm{BPE}$ & 1,34 \\
\hline *fPholiota carbonaria A.H. Sm. & L & & MH 481 & BPE & 1 \\
\hline${ }^{* * f}$ Pholiota lubrica (Pers.) Singer & L & & CTP 405 & BP & 4 \\
\hline *apholiota spumosa (Fr.) Singer & L & & AL 181, GG 24617 & BPE & 1,30 \\
\hline $\begin{array}{l}\text { *f,10 Protostropharia semiglobata (Batsch) Redhead, } \\
\text { Moncalvo \& Vilgalys }\end{array}$ & $\mathrm{F}$ & & IA 813, MH 765, 768 & BPA, BPE, SD & $1,3,6,20,27$ \\
\hline *fStropharia aurantiaca (Cooke) M. Imai & $\mathrm{Hu}$ & & FIGN 34 & BPE & 1 \\
\hline *aStropharia coronilla (Bull.) W. Saunders \& W.G. Sm. & $\mathrm{T}$ & $\mathrm{C}$ & MG 35, OR 804 & BPE & 1,31 \\
\hline \multicolumn{6}{|l|}{ TRICHOLOMATACEAE } \\
\hline${ }^{*}$ Clitocybe gibba (Pers.) P. Kumm. & $\mathrm{T}$ & $\mathrm{C}, \mathrm{M}, \mathrm{Me}$ & CTP 349, DFG 8, RRD 2834 & BPE & $1,3,9,19$ \\
\hline${ }^{10}$ Lepista amara (Alb. \& Schwein.) Maire & $\mathrm{Hu}$ & & AL 178, GN 252, MRSJ 957 & $\mathrm{BPE}, \mathrm{SD}$ & 1,27 \\
\hline *a,fepista nuda (Bull.) Cooke & $\mathrm{Hu}$ & $\mathrm{C}, \mathrm{Me}$ & $\begin{array}{c}\text { CTP 67, 363, IA } 755, \text { OR 1594-A, } \\
4027\end{array}$ & $\mathrm{BMM}, \mathrm{BP}, \mathrm{BPE}$ & $\begin{array}{c}1,2,14,28 \\
34\end{array}$ \\
\hline *f Lepista sordida (Schumach.) Singer & $\mathrm{Hu}$ & C & OV 542, PMM 20 & BPE & 1 \\
\hline${ }^{5}$ Melanoleuca cf. cinereifolia (Bon) Bon & $\mathrm{T}$ & & CA 20, PC 42, SG 24 & BPE & 1 \\
\hline *ePhyllotopsis nidulans (Pers.) Singer & L & & OR 4176-A & BPE & 18 \\
\hline Tricholoma equestre (L.) P. Kumm. & $\mathrm{T}$ & $\mathrm{C}, \mathrm{M}$ & CTP 273, SYR 115 & BPE & 1 \\
\hline${ }^{* a, 10}$ Tricholomopsis rutilans (Schaeff.) Singer & $\mathrm{T}$ & M & CTP 76, OR 1585 & BPE, SD & $1,27,28$ \\
\hline \multicolumn{6}{|l|}{ AURICULARIALES } \\
\hline \multicolumn{6}{|l|}{ AURICULARIACEAE } \\
\hline 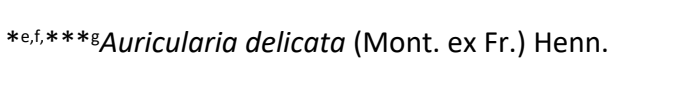 & L & $\mathrm{C}, \mathrm{Me}$ & $\begin{array}{c}\text { SMM 15, OR 4066, 4125, CTP 160, } \\
343\end{array}$ & BMM, BPE & $\begin{array}{c}1,14,17,19 \\
37\end{array}$ \\
\hline${ }^{* e, g, 10}$ Auricularia mesenterica (Dicks.) Pers. & L & $\mathrm{C}, \mathrm{Me}$ & CTP 345, OR 3303 & BMM, BPE, SD & $19,27,37$ \\
\hline $\begin{array}{l}\text { *c,e,f,g,10} \text { Auricularia nigricans (Sw.) Birkebak, Looney \& } \\
\text { Sánchez-García }\end{array}$ & L & C & $\begin{array}{c}A F 6, F S \text { 14, MH 488, 1625, OR } 3017 \\
3075,4126\end{array}$ & $\begin{array}{l}\text { BMM, BPE, BTC, } \\
\text { BTSC, SD }\end{array}$ & $\begin{array}{c}1,14,17,23 \\
24,27,37 \\
39\end{array}$ \\
\hline \multicolumn{6}{|l|}{ EXIDIACEAE } \\
\hline • ${ }^{-}$Ductifera exidioidea Lowy. & L & & CTP 471 & BPE & 1 \\
\hline *c,f,10 Pseudohydnum gelatinosum (Scop.) P. Karst. & L & C & CTP 122, MGTT 654, SYR 120 & BPA, BPE,SD & $1,6,22,27$ \\
\hline
\end{tabular}




\section{BOLETALES}

\section{BOLETACEAE}

${ }^{*}$ A Aureoboletus russellii (Frost) G. Wu \& Zhu L. Yang

${ }^{6}$ Boletus subvelutipes Peck

${ }^{6}$ Boletus variipes Peck

${ }^{10}$ Boletus variipes var. fagicola A.H. Sm. \& Thiers

Buchwaldoboletus hemichrysus (Berk. \& M.A. Curtis) Pilát

Butyriboletus frostii (J.L. Russell) G. Wu, Kuan Zhao \& Zhu L.

Yang

Caloboletus cf. calopus (Pers.) Vizzini

Caloboletus inedulis (Murrill) Vizzini

${ }^{6}$ Cyanoboletus pulverulentus (Opat.) Gelardi, Vizzini \&

Simonini

Leccinum rugosiceps (Peck) Singer

*a,f Neoboletus cf. erythropus (Pers.) C. Hahn

*fPhylloporus rhodoxanthus (Schwein.) Bres.

*a,fStrobilomyces confusus Singer

*aStrobilomyces cf. strobilaceus (Scop.) Berk.

*asutorius eximius (Peck) Halling, Nuhn \& Osmundson

*aTylopilus cf. felleus (Bull.) P. Karst.

Tylopilus ferrugineus (Kuntze) Singer

${ }^{* \mathrm{f}}$ Tylopilus cf. gomezii Singer

${ }^{* f}$ Tylopilus plumbeoviolaceus (Snell \& E.A. Dick) Snell \& E.A. Dick

Xerocomellus chrysenteron (Bull.) Šutara

Xerocomellus truncatus (Singer, Snell \& E.A. Dick) Klofac

\section{BOLETINELLACEAE}

***8Boletinellus merulioides (Schwein.) Murrill

\section{DIPLOCYSTIDIACEAE}

*a,fAstraeus hygrometricus (Pers.) Morgan

\section{GOMPHIDIACEAE}

Chroogomphus rutilus (Schaeff.) O.K. Mill.

$\begin{array}{cc}\mathrm{C}, \mathrm{M} & \text { DRC } 16 \\ \mathrm{C}, \mathrm{M} & \text { LGD } 5905 \\ \mathrm{C}, \mathrm{M}, \mathrm{Me} & \text { MRSJ } 258 \\ \mathrm{M} & \text { OR } 752 \\ \mathrm{M} & \text { MG } 382 \\ \mathrm{C}, \mathrm{M} & \text { OR } 744, \mathrm{~s} . n \\ \mathrm{M}, \mathrm{V} & \text { JG } 5902 \\ \mathrm{M} & \text { SYR } 116 \\ \mathrm{M}, \mathrm{Me} & \\ \mathrm{C}, \mathrm{M}, \mathrm{Me} & \text { LGD } 5349 \\ \mathrm{C}, \mathrm{M}, \mathrm{Me} & \text { MG } 31 \\ \mathrm{M} & \text { OR } 1599 \\ \text { OR } 736\end{array}$

$D R C 16$

\begin{tabular}{|c|c|}
\hline BPE & 1 \\
\hline BPE & 1 \\
\hline SD & 36 \\
\hline BPE, SD & 12,29 \\
\hline BPE & 1 \\
\hline BPE & 1,29 \\
\hline BPE & 1 \\
\hline BPE & 1 \\
\hline BPE & 1 \\
\hline BPE & 1 \\
\hline BPE & 1,28 \\
\hline BPE & 3 \\
\hline BPE & 1,28 \\
\hline BPE & 1,28 \\
\hline BPE & 1,29 \\
\hline BPE & 28 \\
\hline BPE & 1 \\
\hline BPE & 1 \\
\hline BPE & 1 \\
\hline BPE & 1 \\
\hline BPE & 1 \\
\hline $3 \mathrm{MM}, \mathrm{BPE}$ & 1,37 \\
\hline
\end{tabular}




\begin{tabular}{ll}
\hline & Sustrato
\end{tabular}

\section{HYGROPHOROPSIDACEAE}

${ }^{* a, 10}$ Hygrophoropsis aurantiaca (Wulfen) Maire

\section{SCLERODERMATACEAE}

*f,10Pisolithus arhizus (Scop.) Rauschert

*fScleroderma albidum Pat. \& Trab.

*ascleroderma areolatum Ehrenb.

${ }^{10}$ Scleroderma cepa Pers.

Scleroderma michiganense (Guzmán) Guzmán

*escleroderma nitidum Berk.

${ }^{10}$ Scleroderma polyrhizum (J.F. Gmel.) Pers.

${ }^{* * \mathrm{f} S c l e r o d e r m a}$ aff. pseudostipitatum Petch

*f,10Scleroderma texense Berk.

*fScleroderma verrucosum (Bull.) Pers.

\section{SUILLACEAE}

Suillus americanus (Peck) Snell

*asuillus brevipes (Peck) Kuntze

${ }^{*}, 10$ Suillus granulatus (L.) Rousel

Suillus tomentosus Singer

Suillus tomentosus var. discolor A.H. Sm., Thiers \& O.K. Mill.

\section{TAPINELLACEAE}

*a,f,10 Tapinella atrotomentosa (Batsch) Šutara

*e,10Tapinella panuoides (Fr.) E.-J. Gilbert

\section{CANTHARELLALES}

\section{CANTHARELLACEAE}

*a Cantharellus cibarius Fr.

*f Cantharellus cinnabarinus (Schwein.) Schwein.

Craterellus cornucopioides (L.) Pers.

${ }^{*}$ Craterellus tubaeformis (Fr.) Quél.

Pseudocraterellus undulatus (Pers.) Rauschert

\section{CLAVULINACEAE}

*f Clavulina cinerea (Bull.) J. Schröt.

*fClavulina rugosa (Bull.) J. Schröt.
Importancia

Colector

Tipo de vegetación

Localidad
$\mathrm{C}, \mathrm{M}, \mathrm{Me}$

$\mathrm{M}, \mathrm{Me}$

$\mathrm{M}, \mathrm{V}$

$M, M e, V$

$M, V$

$M$

$\mathrm{M}$

$\mathrm{M}$

M

$\mathrm{M}, \mathrm{V}$

$\mathrm{M}, \mathrm{Me}, \mathrm{V}$

C, M

C, M, Me

C, $\mathrm{M}, \mathrm{Me}$

C, $\mathrm{M}$

C, M

L
CTP 73, 512, DGS 353, OR 797

BMM, BP, BPE, SD

$1,2,15,27$

31,34

1,36

BPE, SD

1

MIFL 4

CTP 279, MH 751, RGC 22

SD

EAO s.n.

OR 3968

GG 30562

GG 32654

LSV 415, MLF 783

FJT 583, MLF 794

\section{MACT 15}

RAPC 17

CTP 32, SYR 122

ALSC 1, CTP 64, OR 738

CTP 31, 406

CTP 548, JR s.n., OR 1586, OV s.n. CTP 335, MH 743, OR 2191

C, M, Me
C, M
C, M
C
C

OR 1596, 2176
VMM 15
EL 13, GG 30563
RZV 291

CTP 388, MH 706

\section{BPA, BPE}

SD

BPE

BMM

BPE, SD

BPE

BPE, SD

BPE

BPE

BP

BPE, SD

BPE

$B P, B P E$

BPE, SD

BPA, BPE, SD

$1,9,27,28$

$1,6,18,27$ 
Apéndice 1. Continuación.

Sustrato

Importancia

Colector

Tipo de vegetación

Localidad

\section{HYDNACEAE}

Hydnum repandum $\mathrm{L}$.

C, M, Me

BAAO 561, CTP 454, GL 7

BPA, BP, BPE

$1,4,6$

\section{GEASTRALES}

\section{GEASTRACEAE}

*f Geastrum coronatum Pers.

M

$\mathrm{M}, \mathrm{Me}$

$\mathrm{M}, \mathrm{Me}$

Me

Geastrum triplex Jungh.

*f Geastrum velutinum Morgan.

\section{GLOEOPHYLLALES}

\section{GLOEOPHYLLACEAE}

*e,f Gloeophyllum abietinum (Bull.) P. Karst.

*a Gloeophyllum sepiarium (Wulfen) P. Karst.

*c, $\mathrm{f}$ Gloeophyllum striatum (Fr.) Murrill

\section{GOMPHALES}

\section{CLAVARIADELPHACEAE}

${ }^{*}, 10$ Clavariadelphus truncatus Donk

\section{GOMPHACEAE}

*f Ramaria botrytis s.l. (Pers.) Bourdot

Ramaria flava s.l. (Schaeff.) Quél.

*f Ramaria formosa s.l. (Pers.) Quél.

${ }^{10}$ Ramaria stricta (Pers.) Quél.

*f Turbinellus floccosus (Schwein.) Earle ex Giachini \& Castellano

\section{HYMENOCHAETALES}

\section{HYMENOCHAETACEAE}

${ }^{* f}$ Coltricia cinnamomea (Jacq.) Murrill

*e,fColtricia focicola (Berk. \& M.A. Curtis) Murrill

${ }^{*}$ Coltricia montagnei (Fr.) Murrill

${ }^{* a}$ Coltricia perennis (L.) Murrill

${ }^{7}$ Fuscoporia callimorpha (Lév.) Groposo, Log.-Leite \& Góes-
$\mathrm{T}$

$\mathrm{T}$
CTP 346,461 AT s.n., IA 816, MH 736, OR 802, 4165

FS 11, OV 152

OR 1984

\section{JCNA 6}

$\mathrm{MH} 485$

VMLQ 11

MH 494, VMLQ 14

AT s.n., OR 4118, SYR 119

$\mathrm{MH} 478,794$

CTP 391, DGS 391, MH 744

MAG 14

$\mathrm{M}, \mathrm{Me}$

MH 1500, OR 1601, RIAB 22, SYR 121

GR 1404, RV 1053

$\begin{array}{cc}\text { BPE } & 1 \\ \text { BPE } & 1 \\ \text { BPE, SD } & 1,27 \\ \text { BP, BPE, SD } & 1,7,27\end{array}$

$B M M, B P, B P E$

$1,7,9,15$

BPE

1

BPA, BP, BPE

$1,3,6,7,9$

31,34

1,24

BPE, BTSC

BPE, SD

$12,20,28$

$\begin{array}{cc}\text { BPE } & 1 \\ \text { BPE } & 1 \\ \text { BPE } & 1 \\ \text { BPE, SD } & 1,27 \\ \text { BPA, BP, BPE } & 1,6,7\end{array}$

BPE

1

BMM, BPA, BPE

$1,6,15$

BTC

BPA, BPE

$1,2,6,28$ 
Apéndice 1. Continuación.

\begin{tabular}{|c|c|c|c|c|c|}
\hline & Sustrato & Importancia & Colector & Tipo de vegetación & Localidad \\
\hline Fuscoporia gilva (Schwein.) T. Wagner \& M. Fisch. & $\mathrm{L}, \mathrm{Pp}$ & $\mathrm{D}$ & $M H 685, M J R 8$ & BPE & 1 \\
\hline *eHymenochaete rheicolor (Mont.) Lév. & L & & MH 1639, RZV 310 & BMM & 14 \\
\hline${ }^{* \mathrm{f}}$ Hymenochaete rubiginosa (Dicks.) Lév. & $\mathrm{L}$ & & DM 236, MRSJ 571 & BPE & 1 \\
\hline *f/nonotus cuticularis (Bull.) P. Karst. & L & & SVC 20 & BPE & 1 \\
\hline *f Inonotus fulvomelleus Murrill & $\mathrm{L}$ & & CTP 530, MH 746 & BPA, BPE & 1,6 \\
\hline *fPhellinus badius (Cooke) G. Cunn. & L, Pp & $\mathrm{D}$ & CTP 106, DM 207 & BPE & 1 \\
\hline *a,fPhellinus sarcites (Fr.) Ryvarden & $\mathrm{L}, \mathrm{Pp}$ & $\mathrm{D}$ & CTP 462, OR 1610 & BPE & 1,28 \\
\hline \multicolumn{6}{|l|}{ RICKENELLACEAE } \\
\hline Cotylidia diaphana (Cooke) Lentz & $\mathrm{T}$ & & CT 955, MRSJ 926 & BPE & 1 \\
\hline \multicolumn{6}{|l|}{ HYMENOCHAETALES género incertae sedis } \\
\hline${ }^{* a}$ Trichaptum abietinum (Pers.) Ryvarden & $\mathrm{L}$ & & $\begin{array}{c}\text { BAAO 550, CTP 29, IA 255, MGTT } \\
426, \text { OR } 799\end{array}$ & BPA, BP, BPE & $1,4,6,9,31$ \\
\hline Trichaptum biforme (Fr.) Ryvarden & $\mathrm{L}$ & & CTP 246, 522, MH 739, OR 3068 & BPA, BPE & $1,2,5,6$ \\
\hline${ }^{* \mathrm{f}}$ Trichaptum sector (Ehrenb.) Kreisel & $\mathrm{L}$ & & MH 742, TJRH 8 & BPA, BPE & 1,6 \\
\hline \multicolumn{6}{|l|}{ PHALLALES } \\
\hline \multicolumn{6}{|l|}{ PHALLACEAE } \\
\hline${ }^{* f, 10}$ Phallus hadriani Vent. & $\mathrm{T}$ & & FJT 631 & BPE, SD & 1,27 \\
\hline \multicolumn{6}{|l|}{ POLYPORALES } \\
\hline \multicolumn{6}{|l|}{ DACRYOBOLACEAE } \\
\hline${ }^{* \mathrm{f}}$ Postia caesia (Schrad.) P. Karst. & $\mathrm{L}$ & & CTP 256, 404, SYR 196 & BPA, BP, BPE & $1,4,6$ \\
\hline *fPostia fragilis (Fr.) Jülich & $\mathrm{L}$ & & MH 735, OV 440 & BPA, BPE & 1,6 \\
\hline *a,fSpongiporus floriformis (Quél.) Zmitr. & $\mathrm{L}$ & & MRSJ 180, OR 1605 & BPE & 1,28 \\
\hline \multicolumn{6}{|l|}{ FOMITOPSIDACEAE } \\
\hline${ }^{* a, f}$ Climacocystis borealis (Fr.) Kotl. \& Pouzar & L & C & OR 1594 & BPE & 1,28 \\
\hline${ }^{* \mathrm{f} D a e d a l e a}$ quercina (L.) Pers. & $\mathrm{L}$ & & MEVS 10 & BPE & 1 \\
\hline${ }^{* \mathrm{e}}$ Fomitopsis pinicola (Sw.) P. Karst. & $\mathrm{L}, \mathrm{Pp}$ & $\mathrm{D}, \mathrm{Me}$ & APA 24, MGTT 589, 600 & BMM, BPA, BPE & $1,6,13$ \\
\hline${ }^{*}$ Rhodofomes roseus (Alb. \& Schwein.) Vlasák & $\mathrm{L}$ & & CTP 334 & BPE & 18 \\
\hline *f,g Rhodofomitopsis feei (Fr.) B.K. Cui, M.L. Han \& Y.C. Dai & $\mathrm{L}$ & & CTP 181, MH 741 & BMM, BPA & 6,37 \\
\hline \multicolumn{6}{|l|}{ GANODERMATACEAE } \\
\hline${ }^{* \mathrm{f} G a n o d e r m a ~ a p p l a n a t u m ~(P e r s .) ~ P a t . ~}$ & $\mathrm{~L}, \mathrm{Pp}$ & $\mathrm{D}, \mathrm{Me}$ & MRSJ 562, OR 4110 & BPE & 1 \\
\hline Ganoderma australe (Fr.) Pat. & $\mathrm{L}, \mathrm{Pp}$ & $\mathrm{D}$ & HEFL s.n., JAGV 7 & BPE & 1,2 \\
\hline *a,fGanoderma curtisii (Berk.) Murrill & L, Pp & $\mathrm{D}$ & OR 1603, OV 42 & BPE & 1,28 \\
\hline Ganoderma lobatum (Cooke) G.F. Atk. & $\mathrm{L}, \mathrm{Pp}$ & $\mathrm{D}, \mathrm{Me}$ & $A L 222, M G 30$ & BPE & 1 \\
\hline
\end{tabular}




\begin{tabular}{ll}
\hline & Sustrato \\
\hline GELATOPORIACEAE &
\end{tabular}

$*$ e,***g Gelatoporia dichroa (Fr.) Ginns

\section{LAETIPORACEAE}

${ }^{* f}$ Laetiporus sulphureus (Bull.) Murrill

*a,f,10Phaeolus schweinitzii (Fr.) Pat.

\section{MERIPILACEAE}

*aHydnopolyporus palmatus (Hook.) O. Fidalgo

${ }^{*}$ Rigidoporus lineatus (Pers.) Ryvarden

${ }^{* \mathrm{f} R i g i d o p o r u s ~ m i c r o p o r u s}$ (Sw.) Overeem

\section{MERULIACEAE}

*f Bjerkandera adusta (Willd.) P. Karst.

Cymatoderma caperatum (Berk. \& Mont.) D.A. Reid

*f,10/rpex lacteus (Fr.) Fr.

*e,fPhlebia tremellosa (Schrad.) Nakasone \& Burds.

\section{PHANEROCHAETACEAE}

${ }^{* \mathrm{f}}$ Antrodiella semisupina (Berk. \& M.A. Curtis) Ryvarden

*a,fByssomerulius incarnatus (Schwein.) Gilb.

Phlebiopsis crassa (Lév.) Floudas \& Hibbett

\section{POLYPORACEAE}

${ }^{* f}$ Cerioporus leptocephalus (Jacq.) Zmitr.

${ }^{* \mathrm{f}, * * * \mathrm{~g}}$ Cerioporus varius (Pers.) Zmitr. \& Kovalenko

${ }^{* \mathrm{f} C o r i o l o p s i s ~ b y r s i n a ~(M o n t .) ~ R y v a r d e n ~}$

${ }^{*}$, ${ }^{*}$ Echinochaete brachypora (Mont.) Ryvarden

*a,e,f,gFavolus tenuiculus P. Beauv.

${ }^{* \mathrm{f} F o m e s ~ f a s c i a t u s ~(S w .) ~ C o o k e ~}$

${ }^{*}$ f Funalia caperata (Berk.) Zmitr. \& Malysheva

*d,fFunalia floccosa (Jungh.) Zmitr. \& Malysheva

*fHapalopilus rutilans (Pers.) Murrill

${ }^{* \mathrm{f}}$ Hexagonia cucullata (Mont.) Murrill

$* \mathrm{f}, * * * \mathrm{~g}$ Lentinus arcularius (Batsch) Zmitr.

*a Lentinus badius (Berk.) Berk.
$\mathrm{L}$

Importancia Colector Tipo de vegetación

Localidad

CTP 172, JC 4, LSV 780

BMM, BPE

$1,14,37$

C

Me

Me

C
OV 31-B

MRSJ 241, OV 37

GN 306, IA 808, MH 477

$\mathrm{MH} 750$

MH 752

LSV 644, MH 764, OR 740

OV 40

OV 156,160

BAAO 57, CTP 356, 460, DGS 405, $\mathrm{MH} 760,1635$

\section{BAAO 485, CTP 528, MH 1499}

CTP 380, MACT 11, OR 1606

MRSJ 586

$\mathrm{MH} 730$

EL 14, OR 2979

GEDR 24

CTP 7, OR 743

AGL 8, CTP 170, 185, 479, GN 312, OR 3020,4122

NB s.n.

OR 4395

OR 4397

SMM 24

$\mathrm{CM} 10$

CTP 38, 478, JT 7, OR 3077

OR 1976
BPE

BPE, SD

$1,27,30$

BPE

BPA

BPA

6

$\begin{array}{cc}\text { BPA, BPE } & 1,3,6 \\ \text { BPE } & 1 \\ \text { BPE, SD } & 1,27\end{array}$

BMM, BP,BPA, BPE $1,4,6,14$ 19,26

$\begin{array}{cc}\text { BPE } & 1 \\ \begin{array}{c}\text { BP, BPE } \\ \text { BPE }\end{array} & 1,4,28,35 \\ \text { BPA } & 1 \\ \text { BPE } & 6 \\ \text { BTC } & 1,40 \\ \text { BPE } & 10 \\ & 1,29\end{array}$

BMM, BPE, BTC 1, 2, 17, 30, $37,38,39$

BPE 1

BTC 21

BPE, BTC $\quad 8,21$

BPE 1

BPE 1,3

BMM, BPE $\quad 1,2,5,37$

BPE 


\section{Sustrato}

$* c, f 10, * * *$ g Lentinus crinitus (L.) Fr.

*f Lentinus levis (Berk. \& M.A. Curtis) Murrill

*c,d,e,sLentinus tricholoma (Mont.) Zmitr.

${ }^{10}$ Lenzites betulinus (L.) Fr.

*f Microporellus obovatus (Jungh.) Ryvarden

*e,f Neofavolus alveolaris (DC.) Sotome \& T. Hatt.

Panus conchatus (Bull.) Fr.

***spicipes melanopus (Pers.) Zmitr. \& Kovalenko

Polyporus tuberaster (Jacq. ex Pers.) Fr.

${ }^{* a, 10}$ Pycnoporus sanguineus (L.) Murrill

*d,e,10,***g Trametes elegans (Spreng.) Fr.

*f Trametes hirsuta (Wulfen) Lloyd

*b,e Trametes hirta (P. Beauv.) Zmitr., Wasser \& Ezhov

*a,fTrametes hydnoides (Sw.) Fr.

*f Trametes marianna (Pers.) Ryvarden

*e,f Trametes pavonia (Hook.) Ryvarden

Trametes polyzona (Pers.) Justo

*c,f,g Trametes tenuis (Fr.) Corner

*d,g Trametes variegata (Berk.) Zmitr., Wasser \& Ezhov

Trametes versicolor (L.) Lloyd

${ }^{*}, \mathrm{~d}, \mathrm{~d}, 10$ Trametes villosa (Sw.) Kreisel

*f,***g Trametopsis cervina (Schwein.) Tomšovský

SPARASSIDACEAE

Sparassis crispa (Wulfen) Fr.

RUSSULALES

\section{ALBATRELLACEAE}

Importancia

Colector

Tipo de vegetación

Localidad

CTP 153, FS 19, IF 28, MGG 12, OR BMM, BPE, BTC, 1, 10, 23, 27

3112

BTSC, SD

37,41

C

OR 2187

BPE

1

BAAO 431, CTP 162-A, 244, 353, 569, MGM 18, MH 1623, OR 4390

BMM, BP, BPE, BTC

$1,4,5,14$

$19,21,23$,

37

Me

\section{MH 759 \\ $\mathrm{MH} 693$}

C

ELO 46, OR 4023

LSR 26, LSV 414

CTP 180

LSV 778, MAPC 16

GV 5, MH 757, OR 1602, RIAB 29

MH 1645, MRSJ 585, OR 3306, 4388

CTP 27, 90, 585

AH 23, MRSJ 946, DGS 402

LCNC 14, MH 733, OR 1604

$$
\mathrm{MH} 758
$$

BPRS 9, CLAO 10

Me

LASM 17, MH 1619

AF 4, OR 3018, RISC 2

CTP 184, OR 4394

AR s.n., BAAO 581, CTP 26, MH 716,

$$
1643
$$

CGD 41, DGS 407-A, FS 13, MGTT

705, $M H$ 1628, OR 3096, 4399,

$$
\text { RIAB s.n. }
$$

CTP 109, 193

GN 303

BP 


\section{${ }^{8}$ Laeticutis cristata (Schaeff.) Audet}

${ }^{* a, f}$ Polyporoletus sublividus Snell

\section{AURISCALPIACEAE}

Artomyces pyxidatus (Pers.) Jülich

${ }^{10}$ Auriscalpium vulgare Gray

\section{BONDARZEWIACEAE}

${ }^{* \mathrm{f}}$ Bondarzewia berkeleyi (Fr.) Bondartsev \& Singer

*c,fHeterobasidion annosum (Fr.) Bref.

\section{HERICIACEAE}

Hericium erinaceus (Bull.) Pers.

\section{PENIOPHORACEAE}

${ }^{* f}$ Peniophora albobadia (Schwein.) Boidin

\section{RUSSULACEAE}

*f,10 Lactarius chrysorrheus Fr.

${ }^{*} a, 10$ Lactarius deliciosus s.l. (L.) Gray

*\& Lactarius indigo (Schwein.) Fr.

Lactarius piperatus (L.) Pers.

$* a, 10$ Lactarius torminosus (Schaeff.) Gray

${ }^{*}$ Russula adusta (Pers.) Fr.

*a,fRussula brevipes Peck

${ }^{10}$ Russula cyanoxantha (Schaeff.) Fr.

${ }^{*}$ Russula emetica (Schaeff.) Pers.

*a,e Russula foetens Pers.

*f Russula mexicana Burl.

*a,f Russula rosea Pers.

\section{STEREACEAE}

*f,10Stereum complicatum (Fr.) Fr.

${ }^{10}$ Stereum gausapatum (Fr.) Fr.

*fStereum hirsutum (Willd.) Pers.

*eStereum ochraceoflavum (Schwein.) Sacc.

${ }^{* \mathrm{~d}, 10}$ Stereum ostrea (Blume \& T. Nees) Fr.

Sustrato Importancia

$T$

$M, V$
$C, M, M e$
$C, M, M e$
$C, M$
$M, V$
$C, M$
$C, M, M e$
$C, M$
$M, V$
$M, V$
$C, M$
$C, M$

Me

Me

Colector

Tipo de vegetación

Localidad

OR 809

CTP 21, 483, EPS s.n., IA 812
OMCR 9

MGTT 581, MH 770, SYR 133

LM s.n., OV 421

OR 700

GN 272

CTP 137, DFG 6, OR 747, RIAB 24

CTP 579, MH 720, OR 2977

GN 304

DRDL 11, OR 734, 744-A

$$
\text { CTP } 358
$$

CM 2, OR 737

GN 267, OR 1155

$\mathrm{MH} 473, \mathrm{MHO} 13$

IA 753, MG 32, OR 803, 3962

$$
\text { OR } 739
$$

MRSJ 112, SYR 200

\section{CTP 93, GN 325-A, VMLQ 10}

MGTT 706, MH 754

LSV 772, OR 4161

CTP 591, MH 1640

\section{BPE}

BPA, BPE

1

$1,6,9,22$

BPA, BPE

\section{1,6}

BPE

1

BPE, SD

1,27

BPE, SD

BPA, BPE

$1,2,27,29$

$1,6,40$

30

BPE, SD

$1,3,27,29$

BPE

1

BPE

$1,3,29$

BPE, SD

1,27

BPE

1

BMM, BP, BPE $1,14,31,34$

BPE

3

BPA, BP

6, 34

$\begin{array}{cc}\text { BPE, SD } & 1,27,28,30 \\ \text { BPA, BPE, SD } & 1,6,27,36 \\ \text { BPE } & 1 \\ \text { BMM, BPE } & 1,14\end{array}$

CTP 214, 355, GN 315, IA 810, 823, BMM, BP, BPE, BTC, 1, 3, 5, 14, MRSJ 115, OR 2181, 3993, 4392 
${ }^{* \mathrm{f} S t e r e u m ~ s a n g u i n o l e n t u m ~(A l b . ~ \& ~ S c h w e i n .) ~ F r . ~}$

${ }^{*}$ Xylobolus frustulatus (Pers.) P. Karst.

***8Xylobolus subpileatus (Berk. \& M.A. Curtis) Boidin

\section{SEBACINALES}

\section{SEBACINACEAE}

${ }^{*}$ Helvellosebacina concrescens (Schwein.) Oberw., Garnica

$$
\& \text { K. Riess }
$$

*f Sebacina macrospora (Ellis \& Everh.) Burt

${ }^{* \mathrm{f} S e b a c i n a ~ p u l u l a h u a n a}$ (Pat.) D.P. Rogers

\section{THELEPHORALES}

\section{BANKERACEAE}

${ }^{* \mathrm{f}}$ Hydnellum aurantiacum (Batsch) P. Karst.

*f Phellodon niger (Fr.) P. Karst.

Sarcodon imbricatus (L.) P. Karst.

\section{THELEPHORACEAE}

Thelephora caryophyllea (Schaeff.) Pers.

${ }^{9}$ Thelephora terrestris Ehrh.

\section{DACRYMYCETES}

\section{DACRYMYCETALES}

\section{DACRYMYCETACEAE}

Calocera cornea (Batsch) Fr.

${ }^{* \mathrm{f}}$ Calocera viscosa (Pers.) Fr.

${ }^{* \mathrm{f}}$ Dacrymyces chrysospermus Berk. \& M.A. Curtis

*e,fDacrymyces dictyosporus G.W. Martin

${ }^{* a, e, 10}$ Dacryopinax spathularia (Schwein.) G.W. Martin

\section{PUCCINIOMYCETES}

\section{PUCCINIALES}

\section{CRONARTIACEAE}

Cronartium conigenum Hedgc. \& N.R. Hunt

\section{PUCCINIACEAE}

${ }^{10}$ Aecidium ghesquierei Hendr.

${ }^{10}$ Puccinia heterospora Berk. \& M.A. Curtis

\section{Sustrato Importancia}

L

C

Me

\section{CTP 9}

LSR 16, SG 9

Tipo de vegetación

Localidad

BPE

BPA, BPE

BMM, BPA

1,6

6,38

$$
\mathrm{MH} 486
$$

CTP 30, 352, MGTT 422

CTP 477, GN 305, MH 1620, OR 798
BMM, BPE

1,14

BPE

BPE

1

1

BPE

BPE

BPE

$B P, B P E$

BPE

1, 7, 9

9 
Apéndice 1. Continuación.

\begin{tabular}{|c|c|c|c|c|c|}
\hline & Sustrato & Importancia & Colector & Tipo de vegetación & Localidad \\
\hline \multicolumn{6}{|l|}{ TREMELLOMYCETES } \\
\hline \multicolumn{6}{|l|}{ TREMELLALES } \\
\hline \multicolumn{6}{|l|}{ TREMELLACEAE } \\
\hline Tremella fuciformis Berk. & L & & GN 256, JMRA 36 & BPE & 1,2 \\
\hline *a,e,fTremella mesenterica Retz. & $\mathrm{Fu}$ & Me & CTP 223, 538, MH 1642, OR 1600 & BMM, BPE & $\begin{array}{c}1,5,14,28 \\
30\end{array}$ \\
\hline \multicolumn{6}{|l|}{ USTILAGINOMYCETES } \\
\hline \multicolumn{6}{|l|}{ USTILAGINALES } \\
\hline \multicolumn{6}{|l|}{ USTILAGINACEAE } \\
\hline *cUstilago maydis (DC.) Corda & $\mathrm{Fi}$ & $\mathrm{C}, \mathrm{Me}$ & MB s.n. & $\mathrm{CM}$ & 25 \\
\hline
\end{tabular}


Apéndice 2: Lista de colectores y su abreviatura.

\section{Abreviatura}

\begin{tabular}{|c|c|}
\hline AA & A. Aquino \\
\hline$A C$ & A. Castañeda \\
\hline $\mathrm{AF}$ & A. Figueroa \\
\hline AGJ & A. Gómez-Jiménez \\
\hline AGL & A. Guerrero de León \\
\hline $\mathrm{AH}$ & A. Hermosillo \\
\hline $\mathrm{AL}$ & A. Loeza \\
\hline ALSC & A.L. Sánchez-Casillas \\
\hline APA & A. Palafox Arámbula \\
\hline$A R$ & A. Rocha \\
\hline ASL & A. Sánchez Lira \\
\hline AT & A. Tamayo \\
\hline BAAO & B.A. Arceo-Orozco \\
\hline $\mathrm{BCD}$ & B.C. Durand \\
\hline BEMM & B.E. M. Montes \\
\hline BPRS & B.P. Ramírez Santana \\
\hline $\mathrm{CA}$ & C. Aguilar \\
\hline $\mathrm{CC}$ & C. Cervantes \\
\hline $\mathrm{CDL}$ & C. Díaz-Luna \\
\hline CFMM & C.F. Méndez Martínez \\
\hline CGD & C. Guerrero Díaz \\
\hline CLAO & C.L. Amézola Orozco \\
\hline $\mathrm{CM}$ & C. Motolina \\
\hline CT & C. Téllez \\
\hline CTP & C. Torres-Preciado \\
\hline CVG & C. Valdivia-García \\
\hline DAJR & D.A. Jaimes Rodríguez \\
\hline DCGT & D.C. Gentry Torfer \\
\hline DFG & D. Figueroa-García \\
\hline DGBA & D.G. Brambila Avelar \\
\hline DGS & D. García-Saucedo \\
\hline DM & D. Montañez \\
\hline DRC & D. Rumayor Carrillo \\
\hline DRDL & D.R. Domínguez López \\
\hline EAO & E. Ambríz-Orozco \\
\hline ECE & E. Castro Espíndola \\
\hline EF & E. Fanti \\
\hline EJAB & E.J. Andrade-Bálsamo \\
\hline EL & E. López \\
\hline ELo & E. Loza \\
\hline EPS & E. Pérez-Silva \\
\hline FB & F. Brizuela \\
\hline
\end{tabular}

Abreviatura

Nombre del colector

Nombre del colector

$\begin{array}{cc}\text { FJT } & \text { F.J. Trujillo } \\ \text { FL } & \text { F. Landeros } \\ \text { FLP } & \text { F. López-Pérez } \\ \text { FS } & \text { F. Sánchez } \\ \text { GA } & \text { G. Arias }\end{array}$

GEDR

GG

GGa

$\mathrm{GL}$

GN

GP

GR

GS

GT

GV

HEFL

HR

HRRR

IA

IF

JA

JAGV

JC

JCNA

JEVN

JFN

JG

JMRA

JNR

JPDN

$J P L$

$J R$

JS

JT

KE

KM

LAF

LARR

LASM

LCNC

LERG

\section{F.I. García-Niño \\ F.J. Trujillo \\ F. Landeros \\ F. Sánchez}

G.E. Durán-Rodríguez

G. Guzmán

G. Galindo

G. Leñero

G. Nieves

G. Peralta

G. Rodríguez

G. Santillán

G. Tinoco

G. Velázquez

H.E. Fierros-López

$$
\text { H. Rangel }
$$

H.R. Rueda Rodríguez

I. Álvarez

I. Fortes

J. Aguayo

J.A. García Valle

J. Carranza

J.C. Navarro-Alcalá

J.E. Vázquez Navarrete

J. Fernández Novoa

J. García

J.M. Ramírez Amezcua

J.N. Rose

J.P. Dávila Núñez

J.P. López

J. Ruíz

J. Schöndube

J. Toscano

K. Esqueda

K. Montes

L. Arvizu-Franklin

L.A. Rodríguez Ruvalcaba

L.A. Sánchez Monroy

L.C. Núñez Carrillo

L.E. Ruiz-González 
Apéndice 2: Continuación.

\begin{tabular}{|c|c|c|c|}
\hline Abreviatura & Nombre del colector & Abreviatura & Nombre del colector \\
\hline LGD & L. Guzmán-Dávalos & OV & O. Vargas \\
\hline LJOV & L.J. Ortega Valadez & PC & P. Corona \\
\hline LM & L. Montoya & PMM & P. Munguía-Matute \\
\hline LSR & L. Sánchez-Rivera & RAPC & R.A. Pérez Casanova \\
\hline LSV & L.S. Vázquez & $\mathrm{RB}$ & R. Beas \\
\hline MA & M. Álvarez & $\mathrm{RC}$ & R. Cibrián \\
\hline MACT & M.A. Cortés Tovar & RGC & R. Gutiérrez Cerda \\
\hline MAG & M.A. Guerrero & RIAB & R.I. Alcalá-Briseño \\
\hline MAHR & M.A. Hernández Romo & RISC & R.I. Sandoval Cortés \\
\hline MAPC & M.A. Plasencia Duran & $\mathrm{RL}$ & R. López \\
\hline MB & M. Bustos & RMA & R. Murguía Araiza \\
\hline MCSG & M.C. Sandoval Gutiérrez & RRD & R. Ramírez-Delgadillo \\
\hline MEVS & M.E. Velasco-Sánchez & RV & R. Valenzuela \\
\hline MF & Montoya Fuentes & RZV & R. Zamora-Valdez \\
\hline MG & M. Garza & $\mathrm{SCHR}$ & S.C. Hernández Rodríguez \\
\hline MGG & M.G. Guerrero & SF & S. Franco \\
\hline MGM & M.G. Medina & SG & S. Gallo \\
\hline MGTT & M.G. Torres-Torres & SGCO & S G Canchola Orozco \\
\hline $\mathrm{MH}$ & M. Herrera & & S.G. Canchola Orozco \\
\hline $\mathrm{MHo}$ & M. Homero & SMM & S. Márquez-Marcial \\
\hline MIFL & M.I. Flores Luna & SVC & S. Vargas-Castellanos \\
\hline MJR & M.J. Raygoza & SYR & S.Y. Rubio \\
\hline MLF & M.L. Fierros & TJRH & T.J. Reyes-Hernández \\
\hline MRSJ & M.R. Sánchez-Jácome & TPF & T. Pérez Fiol \\
\hline NB & N. Blanco & VC & V. Chaparro \\
\hline NGRO & N.G. Rojas Osuna & VMLQ & V.M. Lomelí Quintero \\
\hline $\mathrm{OHV}$ & O. Hernández Vargas & VMM & V. Mendoza-Martínez \\
\hline OMCR & O.M. Carrillo Rodríguez & YA & Y. Alquiciras \\
\hline OR & O. Rodríguez & YAGA & Y.A. Guerrero Ahumada \\
\hline
\end{tabular}

